\title{
SHIFTED DERIVED POISSON MANIFOLDS ASSOCIATED WITH LIE PAIRS
}

\author{
RUGGERO BANDIERA, ZHUO CHEN, MATHIEU STIÉNON, AND PING XU
}

\begin{abstract}
Aвstract. We study the shifted analogue of the "Lie-Poisson" construction for $L_{\infty}$ algebroids and we prove that any $L_{\infty}$ algebroid naturally gives rise to shifted derived Poisson manifolds. We also investigate derived Poisson structures from a purely algebraic perspective and, in particular, we establish a homotopy transfer theorem for derived Poisson algebras.

As an application, we prove that, given a Lie pair $(L, A)$, the space tot $\Omega_{A}^{\bullet}\left(\Lambda^{\bullet}(L / A)\right)$ admits a degree $(+1)$ derived Poisson algebra structure with the wedge product as associative multiplication and the ChevalleyEilenberg differential $d_{A}^{\text {Bott }}: \Omega_{A}^{\bullet}\left(\Lambda^{\bullet}(L / A)\right) \rightarrow \Omega_{A}^{\bullet+1}\left(\Lambda^{\bullet}(L / A)\right)$ as unary $L_{\infty}$ bracket. This degree $(+1)$ derived Poisson algebra structure on tot $\Omega_{A}^{\bullet}\left(\Lambda^{\bullet}(L / A)\right)$ is unique up to an isomorphism having the identity map as first Taylor coefficient. Consequently, the Chevalley-Eilenberg hypercohomology $\mathbb{H}\left(\Omega_{A}^{\bullet}\left(\Lambda^{\bullet}(L / A)\right), d_{A}^{\text {Bott }}\right)$ admits a canonical Gerstenhaber algebra structure.
\end{abstract}

\section{Contents}

1. Introduction 2

$\begin{array}{ll}\text { Notations } & 4\end{array}$

2. Derived Poisson algebras 4

2.1. Derived Poisson algebras 4

2.2. Morphisms of derived Poisson algebras 6

2.3. Homotopy transfer for derived Poisson algebras $\quad 8$

3. Shifted derived Poisson manifolds 12

3.1. Shifted derived Poisson manifolds 12

3.2. $L_{\infty}$ algebroids and shifted derived Poisson manifolds $\quad 14$

4. The $(+1)$-shifted derived Poisson algebra arising from a Lie pair 16

4.1. First construction: $L_{\infty}$ algebroid arising from a Lie pair 16

4.2. Second construction: Fedosov dg Lie algebroid arising from a Lie pair 20

4.3. Third construction: Dirac deformation and proof of main theorems 25

4.4. Examples 27

$\begin{array}{lll}\text { Appendix A. } & L_{\infty} \text { algebroids and dg manifolds } & 30\end{array}$

Appendix B. Shifted Poisson algebras $\quad 33$

$\begin{array}{lll}\text { Appendix C. Shifted polyvector fields } & 33\end{array}$

$\begin{array}{ll}\text { Acknowledgments } & 35\end{array}$

References $\quad 35$

Research partially supported by NSFC grant 11471179.

Research partially supported by NSF grants DMS-1406668 and DMS-1707545. 


\section{INTRODUCTION}

The notion of Lie pair is a natural framework encompassing a range of diverse geometric contexts including complex manifolds, foliations, and $\mathfrak{g}$-manifolds (that is, manifolds endowed with an action of a Lie algebra $\mathfrak{g})$. By a Lie pair $(L, A)$, we mean an inclusion $A \hookrightarrow L$ of Lie $\mathbb{K}$-algebroids over a smooth manifold $M$. (Throughout the paper, we use the symbol $\mathbb{K}$ to denote either of the fields $\mathbb{R}$ or $\mathbb{C}$.) Recall that a Lie $\mathbb{K}$ algebroid is a $\mathbb{K}$-vector bundle $L \rightarrow M$, whose space of sections is endowed with a Lie bracket $[-,-]$, together with a bundle map $\rho: L \rightarrow T_{M} \otimes_{\mathbb{R}} \mathbb{K}$ called anchor such that $\rho: \Gamma(L) \rightarrow \mathscr{X}(M) \otimes_{\mathbb{R}} \mathbb{K}$ is a morphism of Lie algebras and $[X, f Y]=f[X, Y]+(\rho(X) f) Y$ for all $X, Y \in \Gamma(L)$ and $f \in C^{\infty}(M, \mathbb{K})$. In other words, a $\mathbb{K}$-vector bundle $L \rightarrow M$ is a Lie $\mathbb{K}$-algebroid if and only if $\Gamma(L)$ is a Lie-Rinehart $\mathbb{K}$ algebra [42] over the commutative ring $C^{\infty}(M, \mathbb{K})$. A Lie pair over the one-point space $M=\{*\}$ is simply a pair of Lie algebras $(\mathfrak{g}, \mathfrak{h})$ with an inclusion of $\mathfrak{h}$ into $\mathfrak{g}$.

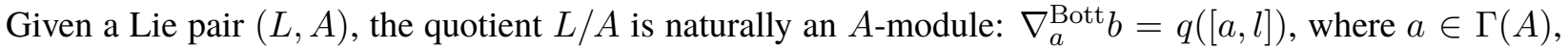
$b \in \Gamma(L / A), q$ denotes the projection $L \rightarrow L / A$, and $l$ is any element of $\Gamma(L)$ such that $q(l)=b$. The flat $A$-connection $\nabla^{\text {Bott }}$ on $L / A$ is known as the Bott connection [6].

Let $\mathscr{X}_{A}$ and $\mathscr{X}_{L}$ denote the differentiable stacks determined by the local Lie groupoids integrating the Lie algebroids $A$ and $L$, respectively. The dg algebra $\left(\operatorname{tot} \Omega_{A}^{\bullet}\left(\Lambda^{\bullet}(L / A)\right), d_{A}^{\text {Bott }}\right)$, where

$$
\operatorname{tot} \Omega_{A}^{\bullet}\left(\Lambda^{\bullet}(L / A)\right)=\bigoplus_{k, l} \Omega_{A}^{k}\left(\Lambda^{l}(L / A)[-l]\right)[k],
$$

can be regarded as the space of formal polyvector fields tangent to the fibers of the differentiable stack fibration $\mathscr{X}_{A} \rightarrow \mathscr{X}_{L}$. For instance, the dg algebra $\left(\operatorname{tot} \Omega_{F}^{\bullet}\left(\Lambda^{\bullet}\left(T_{M} / T_{F}\right)\right), d_{F}^{\text {Bott }}\right)$ associated with the Lie pair $\left(T_{M}, T_{F}\right)$ encoding a foliation $F$ of a smooth manifold $M$ may be thought of as the space of formal polyvector fields on the differentiable stack determined by the holonomy groupoid of the foliation $F$. Therefore, it is natural to ask whether the dg algebra $\left(\operatorname{tot} \Omega_{A}^{\bullet}\left(\Lambda^{\bullet}(L / A)\right), d_{A}^{\text {Bott }}\right)$ admits a " $(+1)$-shifted Lie bracket" compatible with the wedge product - an analogue of the Schouten bracket on the polyvector fields of a smooth manifold. The answer is negative; such a bracket does not exist for arbitrary Lie pairs. However, it turns out that, for every Lie pair $(L, A)$, there does exist a $(+1)$-shifted $L_{\infty}$ algebra structure on tot $\Omega_{A}^{\bullet}\left(\Lambda^{\bullet}(L / A)\right)$, which is compatible with the wedge product in the sense that all its higher brackets satisfy the graded Leibniz rule. This is what we call a degree $(+1)$ derived Poisson algebra, i.e. a "Gerstenhaber algebra up to homotopy."

The main theorem of the paper can be summarized as follows:

Theorem 1.1. Given any Lie pair $(L, A)$, the space tot $\Omega_{A}^{\bullet}\left(\Lambda^{\bullet}(L / A)\right)$ admits a structure of degree $(+1)$ derived Poisson algebra, with the wedge product as associative multiplication and the Chevalley-Eilenberg differential

$$
d_{A}^{\text {Bott }}: \Omega_{A}^{\bullet}\left(\Lambda^{\bullet}(L / A)\right) \rightarrow \Omega_{A}^{\bullet+1}\left(\Lambda^{\bullet}(L / A)\right)
$$

as unary $L_{\infty}$ bracket - the A-module structure on $\Lambda^{\bullet}(L / A)$ is the natural extension of the Bott $A$-connection on $L / A$. This degree $(+1)$ derived Poisson algebra structure is unique up to an isomorphism of degree $(+1)$ derived Poisson algebras having the identity map as first Taylor coefficient.

As an immediate consequence, we obtain the following

Theorem 1.2. Given any Lie pair $(L, A)$, the Chevalley-Eilenberg hypercohomology

$$
\mathbb{H}\left(\Omega_{A}^{\bullet}\left(\Lambda^{\bullet}(L / A)\right), d_{A}^{\text {Bott }}\right)
$$

admits a canonical Gerstenhaber algebra structure.

In [2], an $L_{\infty}$ [1] algebra structure on tot $\Omega_{A}^{\bullet}\left(\Lambda^{\bullet}(L / A)\right)$ was constructed explicitly via Fedosov dg Lie algebroids - see Section 4.2. Its construction relies on the choice of additional geometric data: a splitting of 
the short exact sequence $0 \rightarrow A \rightarrow L \rightarrow L / A \rightarrow 0$ and a torsion-free $L$-connection $\nabla$ on $L / A$ extending the Bott $A$-connection. A similar construction for polydifferential operators rather than polyvector fields was described in [2] as well. Understanding the extent to which the resulting $L_{\infty}[1]$ algebra structures on tot $\Omega_{A}^{\bullet}\left(\Lambda^{\bullet}(L / A)\right)$ and its polydifferential operator counterpart depend on the geometric data chosen is an issue that was addressed in [2].

In this paper, we propose a more direct approach to the two-fold problem of existence and uniqueness of a structure of derived Poisson algebra of degree $(+1)$ on tot $\Omega_{A}^{\bullet}\left(\Lambda^{\bullet}(L / A)\right)$ : we describe two new ways of constructing such structures - one involves $L_{\infty}$ algebroids while the other involves deformations of Dirac structures - and we prove that all three approaches yield exactly the same degree $(+1)$ derived Poisson algebra structure on tot $\Omega_{A}^{\bullet}\left(\Lambda^{\bullet}(L / A)\right)$ - see Section 4.3. The uniqueness of the degree $(+1)$ derived Poisson algebra structure in Theorem 1.1 then follows from a standard result of Ševera on deformations of Dirac structures [50, 49].

Let us briefly recall the construction via deformations of Dirac structures. The deformation of Dirac structures were investigated about 15 years ago by Ševera, Roytenberg, and many others [50, 43]. Given a Courant algebroid $E$ of signature $(n, n)$, the deformations of a Dirac structure $D$ in $E$ are governed by an $L_{\infty}$ algebra structure on $\Gamma\left(\Lambda^{\bullet} D^{\vee}\right)$, which is in fact a degree $(-1)$ derived Poisson algebra unique up to isomorphism [50]. Here $\Gamma\left(\Lambda^{\bullet} D^{\vee}\right)=\bigoplus_{l} \Gamma\left(\Lambda^{l} D^{\vee}\right)[-l]$. Now, given a Lie pair $(L, A)$, it is well known that $E=L \oplus L^{\vee}$ is a Courant algebroid of signature $(n, n)$ and $D=A \oplus A^{\perp}$ is a Dirac structure in $E$ [32]. It is easy to see that the isomorphism $\Gamma\left(\Lambda^{m} D^{\vee}\right) \cong \bigoplus_{k+l=m} \Omega_{A}^{k}\left(\Lambda^{l}(L / A)\right)$ identifies the first $L_{\infty}$ bracket on $\Gamma\left(\Lambda^{\bullet} D^{\vee}\right)$ with the Chevalley-Eilenberg differential $d_{A}^{\text {Bott }}: \Omega_{A}^{\bullet}\left(\Lambda^{\bullet}(L / A)\right) \rightarrow \Omega_{A}^{\bullet+1}\left(\Lambda^{\bullet}(L / A)\right)$. Thus one obtains a degree $(-1)$ derived Poisson algebra structure on the dg algebra $\left(\bigoplus_{k+l=\bullet} \Omega_{A}^{k}\left(\Lambda^{l}(L / A)\right)[-k-l], d_{A}^{\text {Bott }}\right)$. Shifting the graduation, we obtain a structure of derived Poisson algebra of degree $(+1)$ on tot $\Omega_{A}^{\bullet}\left(\Lambda^{\bullet}(L / A)\right)=$ $\bigoplus_{k, l} \Omega_{A}^{k}\left(\Lambda^{l}(L / A)\right)[-k+l]$.

Next, we proceed to outline the construction of the degree $(+1)$ derived Poisson algebra structure on tot $\Omega_{A}^{\bullet}\left(\Lambda^{\bullet}(L / A)\right)$ via $L_{\infty}$ algebroids.

An $L_{\infty}$ algebroid is a vector bundle $\mathcal{L} \rightarrow \mathcal{M}$ of $\mathbb{Z}$-graded manifolds endowed with (1) a sequence $\left(\lambda_{l}\right)_{l \geq 1}$ of maps $\lambda_{l}: \Lambda^{l} \Gamma(\mathcal{L}) \rightarrow \Gamma(\mathcal{L})[2-l]$, called multi-brackets, that determine a structure of $L_{\infty}$ algebra on $\Gamma(\mathcal{L})$ and (2) a sequence $\left(\rho_{l}\right)_{l \geq 0}$ of bundle maps $\rho_{l}: \Lambda^{l} \mathcal{L} \rightarrow T_{\mathcal{M}} \otimes_{\mathbb{R}} \mathbb{K}[1-l]$, called anchor maps, that determine a morphism of $L_{\infty}$ algebras from $\Gamma(\mathcal{L})$ to $\mathscr{X}(\mathcal{M}) \otimes_{\mathbb{R}} \mathbb{K}$. The $L_{\infty}$-brackets $\lambda_{l}$ and the anchor maps $\rho_{l}$ must satisfy the usual compatibility condition $[18,8,52]$.

There exists an equivalent and more compact definition of $L_{\infty}$ algebroids à la Vaĭntrob via dg manifolds [51], which we will recall briefly. A dg manifold is a $\mathbb{Z}$-graded manifold $\mathcal{M}$ together with a homological vector field, i.e. vector field $Q \in \mathscr{X}(\mathcal{M})$ of degree $(+1)$ satisfying $Q^{2}=0$. An $L_{\infty}$ algebroid is a vector bundle $\mathcal{L} \rightarrow \mathcal{M}$ of $\mathbb{Z}$-graded manifolds together with a homological vector field $Q$ on $\mathcal{L}[1]$ tangent to the zero section $\mathcal{M} \subset \mathcal{L}[1]-$ see Proposition A.1.

It turns out that $L_{\infty}$ algebroids are closely related to shifted derived $C^{\infty}$-Poisson manifolds in the sense of Pridham [39]. Let $\hat{\mathscr{X}}_{\text {poly }}^{\bullet}(\mathcal{M}, n)$ denote the completion of the space of $n$-shifted polyvector fields on $\mathcal{M}-$ see Appendix $\mathrm{C}$ for details. A $(-k)$-shifted derived Poisson manifold (see Definition 3.1) can be thought of as a dg manifold $(\mathcal{M}, Q)$ equipped with a formal series $\pi=\sum_{l=2}^{\infty} \pi_{l}$ of $(k-2)$-shifted polyvector fields, with $\pi_{l} \in \hat{\mathscr{X}}_{\text {poly }}^{l}(\mathcal{M}, k-2)$ of degree $(+1)$ in $\hat{\mathscr{X}}_{\text {poly }}^{\bullet}(\mathcal{M}, k-2)[k-1]$, satisfying the Maurer-Cartan equation $[Q, \pi]+\frac{1}{2}[\pi, \pi]=0$. The well known "Lie-Poisson" construction admits the following analogue in the "shifted derived" context.

Theorem 1.3. Let $\mathcal{L} \rightarrow \mathcal{M}$ be a vector bundle of $\mathbb{Z}$-graded manifolds, and let $k \in \mathbb{Z}$ be a fixed integer. The following statements are equivalent.

(1) The vector bundle $\mathcal{L} \rightarrow \mathcal{M}$ is an $L_{\infty}$ algebroid.

(2) The space $\Gamma(\hat{S}(\mathcal{L}[k]))$ is a degree $k$ derived Poisson algebra with l-th bracket of weight $(1-l)$. 
(3) The graded manifold $\mathcal{L}^{\vee}[-k]$ is a $(-k)$-shifted derived Poisson manifold and the weight of the lthbracket on $C^{\infty}\left(\mathcal{L}^{\vee}[-k]\right)$ is $(1-l)$.

Now, going back to a Lie pair $(L, A)$, it is easily seen that, once a splitting of the short exact $0 \rightarrow A \rightarrow L \rightarrow$ $L / A \rightarrow 0$ has been chosen, the graded vector bundle $A[1] \times L / A \rightarrow A[1]$ acquires a natural $L_{\infty}$ algebroid structure. This $L_{\infty}$ algebroid was studied by Vitagliano in the special case of a Lie pair corresponding to a foliation [52]. Applying Theorem 1.3 to this $L_{\infty}$ algebroid and the integer $k=+1$ yields a degree $(+1)$ derived Poisson algebra structure on tot $\Omega_{A}^{\bullet}\left(\Lambda^{\bullet}(L / A)\right)$.

The discussion of the constructions outlined above occupies Sections 3 and 4. Section 2 is devoted to the study of derived Poisson structures from a purely algebraic perspective and contains, in particular, the proof of a homotopy transfer result for derived Poisson algebras.

We would like to point out that, in the context of $\mathbb{Z}_{2}$-grading, "shifted derived Poisson algebras" were studied by Voronov [55, 56], Khudaverdian-Voronov [24], and Bruce [8], who called them homotopy Poisson algebras and homotopy Schouten algebras, respectively. Derived Poisson algebras of degree 0 were also studied by Oh-Park [38] and Cattaneo-Felder [9], who called them $P_{\infty}$ algebras. See also [39, 3]. For recent developments, see [58], [25] and [57]. References [29, 46, 4] are also related to the present paper.

Notations. In this paper, unless specified otherwise, graded means $\mathbb{Z}$-graded. Given a graded vector space $V=\bigoplus_{n \in \mathbb{Z}} V^{n}$, we say that an element $v \in V^{n}$ has degree $n$ and we write $|v|=n$. Given a graded vector space $V=\bigoplus_{n \in \mathbb{Z}} V^{n}$, the symbol $V[k]$ denotes the graded vector space obtained from $V$ by shifting the graduation according to the rule $(V[k])^{n}=V^{n+k}$. We write $|v|^{[k]}$ to denote the degree of $v$ when regarded as an element of $V[-k]$. Therefore,

$$
|v|^{[k]}=|v|+k .
$$

The dual $V^{\vee}$ of a graded vector space $V$ is graded according to the rule $\left(V^{\vee}\right)^{n}=\left(V^{-n}\right)^{\vee}$.

Likewise, if $E=\bigoplus_{n \in \mathbb{Z}} E^{n}$ is a graded vector bundle over a manifold $M, E[k]$ denotes the graded vector bundle obtained by shifting the graduation of the fibers of $E$ according to the above rule.

Given a vector space $V$, the symbol $\hat{S}(V)$ denotes the m-adic completion of the symmetric algebra $S(V)$, where $\mathfrak{m}$ is the ideal of $S(V)$ generated by $V$. Thus, $\hat{S}(V)=\prod_{p=0}^{\infty} S^{p}(V)$. The symbol $\bar{S}(V)$ denotes the reduced symmetric algebra of $V$, i.e. $\bar{S}(V)=\bigoplus_{p=1}^{\infty} S^{p}(V)$.

The Koszul sign $\epsilon\left(\sigma ; v_{1}, \cdots, v_{p}\right)$ of a permutation $\sigma \in \mathfrak{S}_{p}$ of $p$ homogeneous vectors $v_{1}, v_{2}, \ldots, v_{p}$ of a graded vector space $V$ - which will be abbreviated as $\epsilon(\sigma)$ - is determined by the relation

$$
v_{\sigma(1)} \odot v_{\sigma(2)} \odot \cdots \odot v_{\sigma(p)}=\epsilon\left(\sigma ; v_{1}, \cdots, v_{p}\right) v_{1} \odot v_{2} \odot \cdots \odot v_{p},
$$

where $\odot$ denotes the multiplication in the symmetric algebra $S(V)$.

An $(r, s)$-shuffle is a permutation $\sigma$ of the set $\{1,2, \cdots, r+s\}$ such that $\sigma(1) \leq \sigma(2) \leq \cdots \leq \sigma(r)$ and $\sigma(r+1) \leq \sigma(r+2) \leq \cdots \leq \sigma(r+s)$. We write $\operatorname{Sh}(r, s)$ to denote the set of $(r, s)$-shuffles.

\section{Derived Poisson algebras}

2.1. Derived Poisson algebras. Let $k \in \mathbb{Z}$ be a fixed integer, and $\mathbb{K}$ a field of characteristic zero.

Given a $\mathbb{Z}$-graded $\mathbb{K}$-vector space $V=\bigoplus_{k \in \mathbb{Z}} V^{i}$, we denote by $\left(\bar{S}(V)=\oplus_{n \geq 1} V^{\odot n}, \Delta\right)$ the reduced symmetric tensor coalgebra on $V$. This is the cofree cocommutative locally conilpotent coalgebra generated by $V$. In particular, every coderivation $Q: \bar{S}(V) \rightarrow \bar{S}(V)$ is completely determined by its corestriction $p \circ Q=: q=:\left(q_{1}, \ldots, q_{n}, \ldots\right)$, where $p: \bar{S}(V) \rightarrow V$ denotes the canonical projection. The sequence of maps $q_{n}: V^{\odot n} \rightarrow V, n \geq 1$, are called the Taylor coefficients of $Q$. Similarly, given another graded space $W$, every morphism of coalgebras $F: \bar{S}(W) \rightarrow \bar{S}(V)$ is completely determined by its corestriction 
$p \circ F=f=\left(f_{1}, \ldots, f_{n}, \ldots\right)$. Again we call the sequence of maps $f_{n}: W^{\odot n} \rightarrow V, n \geq 1$, the Taylor coefficients of $F$.

Definition 2.1. A degree $k$ derived Poisson algebra is a $\mathbb{Z}$-graded commutative $\mathbb{K}$-algebra $A=\bigoplus_{k \in \mathbb{Z}} A^{i}$ together with a degree $(+1)$ coderivation

$$
Q: \bar{S}(A[1-k]) \rightarrow \bar{S}(A[1-k])[1]
$$

of the reduced symmetric tensor coalgebra $(\bar{S}(A[1-k]), \Delta)$ satisfying

(1) the cohomological condition $Q \circ Q=0$; and

(2) the Leibniz rule

$$
q_{n}\left(a_{1}, \ldots, a_{n-1}, a_{n} a_{n}^{\prime}\right)=q_{n}\left(a_{1}, \ldots, a_{n-1}, a_{n}\right) a_{n}^{\prime}+(-1)^{\epsilon} a_{n} q_{n}\left(a_{1}, \ldots, a_{n-1}, a_{n}^{\prime}\right)
$$

with $\epsilon=\left\{(2-n)+\left(\alpha_{1}+\cdots+\alpha_{n-1}\right)+k(n-1)\right\} \alpha_{n}$, for all $n \geq 1$ and $a_{1} \in A^{\alpha_{1}}, a_{2} \in A^{\alpha_{2}}$, $\ldots, a_{n} \in A^{\alpha_{n}}, a_{n}^{\prime} \in A$. An equivalent expression is

$$
q_{n}\left(a_{1}, \ldots, a_{n-1}, a_{n} a_{n}^{\prime}\right)=q_{n}\left(a_{1}, \ldots, a_{n-1}, a_{n}\right) a_{n}^{\prime}+(-1)^{\alpha_{n} \alpha_{n}^{\prime}} q_{n}\left(a_{1}, \ldots, a_{n-1}, a_{n}^{\prime}\right) a_{n}
$$

for all $a_{1}, \ldots, a_{n-1} \in A, a_{n} \in A^{\alpha_{n}}, a_{n}^{\prime} \in A^{\alpha_{n}^{\prime}}$.

To understand the meaning of Equation (1), and in particular the sign $(-1)^{\epsilon}$ of its last term, observe that, if $a_{1} \in A^{\alpha_{1}}, a_{2} \in A^{\alpha_{2}}, \cdots, a_{n} \in A^{\alpha_{n}}$, or equivalently $a_{1} \in(A[1-k])^{\alpha_{1}-1+k}, a_{2} \in(A[1-k])^{\alpha_{2}-1+k}, \cdots, a_{n} \in$ $(A[1-k])^{\alpha_{n}-1+k}$, then we have

$$
q_{n}\left(a_{1}, a_{2}, \ldots, a_{n}\right) \in(A[1-k])^{1+\left(\alpha_{1}-1+k\right)+\left(\alpha_{2}-1+k\right)+\cdots+\left(\alpha_{n}-1+k\right)},
$$

or equivalently

$$
q_{n}\left(a_{1}, a_{2}, \ldots, a_{n}\right) \in A^{(1-k)+1+\left(\alpha_{1}-1+k\right)+\left(\alpha_{2}-1+k\right)+\cdots+\left(\alpha_{n}-1+k\right)} .
$$

Therefore,

$$
A \ni x \mapsto q_{n}\left(a_{1}, \ldots, a_{n-1}, x\right) \in A
$$

is an operator on $A$ of degree

$$
1+\left(\alpha_{1}-1+k\right)+\cdots+\left(\alpha_{n-1}-1+k\right)=(2-n)+\left(\alpha_{1}+\cdots+\alpha_{n-1}\right)+k(n-1) .
$$

Equation (1) means that this operator is a graded derivation on $A$.

An equivalent description of the above definition is the following:

Definition 2.2. A degree $k$ derived Poisson algebra is a $\mathbb{Z}$-graded commutative algebra $A=\bigoplus_{i \in \mathbb{Z}} A^{i}$ (over a field $\mathbb{K}$ of characteristic zero) endowed with a family of $\mathbb{K}$-multilinear maps $\lambda_{n}: A^{\otimes n} \rightarrow A$ of degree $k(n-1)+2-n,(n=1,2, \cdots)$, defining an $L_{\infty}$ algebra structure on $A[-k]$ such that for all $a_{1}, \cdots, a_{n-1} \in$ $A$, the map

$$
A \rightarrow A, \quad a \mapsto \lambda_{n}\left(a_{1}, \cdots, a_{n-1}, a\right)
$$

is a graded derivation.

We note that the maps $\lambda_{n}$ and $q_{n}$ from the previous definitions are related by décalage, that is,

$$
q_{n}\left(a_{1}, \ldots, a_{n}\right)=(-1)^{\epsilon} \lambda_{n}\left(a_{1}, \ldots, a_{n}\right)
$$

for all $n \geq 1$ and $a_{1} \in A^{\alpha_{1}}, \ldots, a_{n} \in A^{\alpha_{n}}$, where $\epsilon=\sum_{i=1}^{n}(n-i)\left(\alpha_{i}+k\right)$. In particular, while the $q_{n}$ can be considered as degree one graded symmetric maps $q_{n}: A[1-k]^{\odot n} \rightarrow A[1-k]$, the $\lambda_{n}$ can be considered as degree $2-n$ graded antisymmetric maps $\lambda_{n}: A[-k]^{\wedge n} \rightarrow A[-k]$. In the sequel, the structure maps $\lambda_{n}$ are also denoted by $\{\cdots\}_{n}$, just as the usual Poisson brackets.

A degree $k$ Poisson algebra (see Appendix B) is a degree $k$ derived Poisson algebra, where the only nontrivial bracket is the binary bracket $\lambda_{2}=\{-,-\}$. 
Remark 2.3. In the context of $\mathbb{Z}_{2}$-grading, Definition 2.2 reduces to homotopy Poisson algebras and homotopy Schouten algebras studied by Voronov [55, 56], Khudaverdian-Voronov [24], and Bruce [8].

Derived Poisson algebras of degree 0 were also studied by Oh-Park [38] and Cattaneo-Felder [9], who called them $P_{\infty}$ algebras.

Given a degree $k$ derived Poisson algebra $\left(A,\left(\lambda_{l}\right)_{l \geq 1}\right)$, we have a cochain complex $\lambda_{1}: A^{\bullet} \rightarrow A^{\bullet+1}$. The binary bracket $\lambda_{2}$ is of degree $k$, and satisfies the Jacobi identity up to homotopy. The following is thus immediate.

Proposition 2.4. If $A$ is a degree $k$ derived Poisson algebra, then the binary bracket $\lambda_{2}$ induces, on the cohomology groups $\mathbb{H}\left(A, \lambda_{1}\right)$, a degree $k$ Poisson algebra structure.

\subsection{Morphisms of derived Poisson algebras.}

Definition 2.5. Let $\left(A, Q_{A}\right)$ and $\left(B, Q_{B}\right)$ be degree $k$ derived Poisson algebras: here $Q_{A}$ denotes the corresponding coderivation on $\bar{S}(A[1-k])$, similarly for $Q_{B}$, see Definition 2.1. A morphism $f_{\infty}=$ $\left(f_{1}, \ldots, f_{n}, \ldots\right): B \rightarrow A$ of derived Poisson algebras is a collection of degree $(k-1)(n-1)$ maps $f_{n}: B^{\otimes n} \rightarrow A, n \geq 1$, such that

(1) If we regard the $f_{n}$ as degree 0 maps $B[1-k]^{\otimes n} \rightarrow A[1-k]$ they are graded symmetric, and the unique morphism $F: \bar{S}(B[1-k]) \rightarrow \bar{S}(A[1-k])$ of coalgebras with corestriction $p \circ F=f_{\infty}$ satisfies $F \circ Q_{B}=Q_{A} \circ F$. In other words, this says that the maps $f_{n}$ induce (after décalage) an $L_{\infty}$ morphism from $B[-k]$ to $A[-k]$.

(2) The following relation is satisfied for all $n \geq 0$ and $x_{1}, \ldots, x_{n}, y, z \in B$ :

$$
f_{n+1}\left(x_{1}, \ldots, x_{n}, y z\right)=\sum_{i=0}^{n} \sum_{\sigma \in \operatorname{Sh}(i, n-i)}(-1)^{\diamond} f_{i+1}\left(x_{\sigma(1)}, \ldots, x_{\sigma(i)}, y\right) f_{n-i+1}\left(x_{\sigma(i+1)}, \ldots, x_{\sigma(n)}, z\right) \text {, }
$$

where $\diamond=\epsilon\left(\sigma ; x_{1}, x_{2}, \cdots, x_{n}\right)+|y|\left((n-i)(k-1)+\left|x_{\sigma(i+1)}\right|+\cdots+\left|x_{\sigma(n)}\right|\right)$, and the Koszul sign $\epsilon\left(\sigma ; x_{1}, x_{2}, \cdots, x_{n}\right)$ associated to the permutation $\sigma$ is computed by regarding $x_{1}, \ldots, x_{n}$ as elements of $B[1-k]$, that is, of degrees $\left|x_{1}\right|+k-1, \ldots,\left|x_{n}\right|+k-1$.

In particular, for $n=0$, this says that $f_{1}: B \rightarrow A$ is a morphism of associative algebras, and for $n=1$, it says that $f_{2}: B^{\otimes 2} \rightarrow A$ is an $f_{1}$-biderivation.

Remark 2.6. Since $S(B[1-k])$ is a graded cocommutative coalgebra via the unshuffle coproduct $\Delta$ and $A$ is a graded commutative algebra with product $m_{A}: A^{\otimes 2} \rightarrow A$, the space $\operatorname{Hom}(S(B[1-k]), A)$ is a graded commutative algebra via the convolution product $f \star g=m_{A} \circ(f \otimes g) \circ \Delta$. Given $y \in B$, we denote by $f(\ldots, y)$ the map

$$
\begin{aligned}
& f(\ldots, y): S(B[1-k]) \rightarrow A, \\
& x_{1} \odot \cdots \odot x_{n} \mapsto(-1)^{\left(n(k-1)+\left|x_{1}\right|+\cdots+\left|x_{n}\right|\right)|y|} f_{n+1}\left(x_{1}, \ldots, x_{n}, y\right),
\end{aligned}
$$

where $x_{i} \in B^{\left|x_{i}\right|}=B[1-k]^{\left|x_{i}\right|+k-1}, i=1, \ldots, n$. Then Equation (2) is equivalent to

$$
f(\ldots, y z)=f(\ldots, y) \star f(\ldots, z) \text {. }
$$

In other words, $f$ is a morphism of degree $k$ derived Poisson algebras if and only if

$$
\begin{aligned}
& \left(B, m_{B}\right) \rightarrow(\operatorname{Hom}(S(B[1-k]), A), \star), \\
& y \mapsto f(\ldots, y)
\end{aligned}
$$

is a morphism of graded algebras.

The following proposition can be proved by a tedious direct computation, which we omit.

Proposition 2.7. With the above definition of morphisms, degree $k$ derived Poisson algebras form a category. 
The following Proposition 2.8 justifies the previous definition in the framework of deformation theory. Given a graded algebra $\left(A, m_{A}\right)$, we denote by $L=\operatorname{Coder}(\bar{S}(A[1-k]))$ the graded Lie algebra of coderivations of the reduced symmetric coalgebra $\bar{S}(A[1-k])$. From the point of view of deformation theory (cf. [36]), this graded Lie algebra controls the deformations of the trivial $L_{\infty}$ algebra structure on $A[-k]$. In other words, the set $\mathrm{MC}(L)$ of Maurer-Cartan elements of $L$, that is, the set of solutions $Q \in L^{1}$ of the MaurerCartan equation $[Q, Q]=0$, is in bijective correspondence with the set of $L_{\infty}$ algebra structures on $A[-k]$. Furthermore, the (formal) exponential group $\exp \left(L^{0}\right)=\left\{e^{R}\right\}_{R \in L^{0}}$ acts on the set $\mathrm{MC}(L)$ via conjugation $Q \mapsto e^{-R} Q e^{R}$, and the corresponding set of orbits parameterizes $L_{\infty}$ algebra structures on $A[-k]$ up to $L_{\infty}$ isomorphism. An easy computation shows that the subspace $M \subset L$, spanned by those coderivations $Q$ whose Taylor coefficients $p \circ Q=\left(q_{1}, \ldots, q_{n}, \ldots\right)$ are multiderivations, i.e., they satisfy the Leibniz rule (1) from Definition 2.1, is a graded Lie subalgebra. Moreover, it is clear that the Maurer-Cartan elements in $M$ are precisely the degree $k$ derived Poisson algebra structures on $\left(A, m_{A}\right)$. In the following Proposition 2.8, we show that the (formal) exponential group $\exp \left(M^{0}\right)$ is precisely the group of coalgebra automorphisms $F: \bar{S}(A[1-k]) \rightarrow \bar{S}(A[1-k])$ whose Taylor coefficients $p \circ F=\left(f_{1}, \ldots, f_{n}, \ldots\right)$ satisfy Equation (2).

In order to avoid convergence issue, we proceed formally. We denote by $\mathbb{K}[[t]]$ the algebra of formal power series, by $A[[t]]$ the algebra $A \otimes_{\mathbb{K}} \mathbb{K}[[t]]$ of formal power series with coefficients in $A$, and by $\bar{S}_{\mathbb{K}[[t]]}(A[[t]][1-k])$ the reduced symmetric $\mathbb{K}[[t]]$-coalgebra over the $\mathbb{K}[[t]]$-module $A[[t]][1-k]$. Given a degree zero coderivation $R \in L^{0}$, we consider the associated formal flow $e^{t R}:=F^{t}: \bar{S}_{\mathbb{K}[[t]]}(A[[t]][1-k]) \rightarrow \bar{S}_{\mathbb{K}[[t]]}(A[[t]][1-k])$. It is a well defined $\mathbb{K}[[t]]$-linear coalgebra automorphism.

Proposition 2.8. Given $R \in L^{0}, p \circ R=\left(r_{1}, \ldots, r_{n}, \ldots\right)$ as above, then the Taylor coefficients $f_{n}^{t}$ of the formal flow

$$
e^{t R}:=F^{t}, \quad p \circ F^{t}=\left(f_{1}^{t}, \ldots, f_{n}^{t}, \ldots\right)
$$

satisfy Equation (2) if and only if all Taylor coefficients $r_{n}: A^{\otimes n} \rightarrow A, n \geq 1$, are multi-derivations, that is, if and only if $R \in M^{0}$.

Proof. For notational simplicity, in the following computations we abbreviate equations such as Equation (2) by omitting the $x_{1}, \ldots, x_{n}$ arguments, and by writing $\pm_{K}$ instead of the appropriate Koszul sign (signs were made precise in Definition 2.5). For instance, Equation (2) becomes

$$
f_{n+1}^{t}(\ldots, y z)=\sum_{i=0}^{n} \sum_{\sigma \in \operatorname{Sh}(i, n-i)} \pm_{K} f_{i+1}^{t}(\ldots, y) f_{n-i+1}^{t}(\ldots, z) .
$$

Having set these notations, we proceed with the proof. One implication is easy: assuming Equation (2) is satisfied, then for all $x_{1}, \ldots, x_{n}, y, z \in A$,

$$
\begin{gathered}
r_{n+1}(\ldots, y z)=\left.\frac{d}{d t} f_{n+1}^{t}(\ldots, y z)\right|_{t=0}=\sum_{i=0}^{n} \sum_{\sigma \in \operatorname{Sh}(i, n-i)} \frac{d}{d t}\left( \pm_{K} f_{i+1}^{t}(\ldots, y) f_{n-i+1}^{t}(\ldots, z)\right)_{\mid t=0}= \\
=\sum_{i=0}^{n} \sum_{\sigma \in \operatorname{Sh}(i, n-i)} \pm_{K} \frac{d}{d t} f_{i+1}^{t}(\ldots, y)_{\mid t=0} f_{n-i+1}^{0}(\ldots, z) \pm_{K} f_{i+1}^{0}(\ldots, y) \frac{d}{d t} f_{n-i+1}^{t}(\ldots, z)_{\mid t=0}= \\
=r_{n+1}(\ldots, y) z \pm_{K} y r_{n+1}(\ldots, z),
\end{gathered}
$$

since $p \circ F^{0}=(\mathrm{id}, 0, \ldots, 0, \ldots)$, i.e. $f_{1}^{0}=\mathrm{id}$ and $f_{k}^{0}=0$ for $k \geq 2$. This shows that $r_{n+1}$ is a multiderivation for all $n \geq 0$.

We turn to the other implication. Since $r_{1}$ is an algebra derivation, $f_{1}^{t}=e^{t r_{1}}: A[[t]] \rightarrow A[[t]]$ is an algebra morphism. Let $n \geq 1$, and fix $x_{1}, \ldots, x_{n}, y, z \in A$. We consider the formal power series

$$
A[[t]] \ni \xi(t):=f_{n+1}^{t}(\ldots, y z)-\sum_{i=0}^{n} \sum_{\sigma \in \operatorname{Sh}(i, n-i)} \pm_{K} f_{i+1}^{t}(\ldots, y) f_{n-i+1}^{t}(\ldots, z) .
$$


We need to prove that $\xi(t)=0$. As before, since $F^{0}=(\mathrm{id}, 0, \cdots, 0, \cdots)$, we see that $\xi(0)=0$. Since $F^{t}=e^{t R}$, we have $\frac{d}{d t} F^{t}=R F^{t}$. Using induction on $n$, we have

$$
\begin{aligned}
& \frac{d}{d t} f_{n+1}^{t}(\ldots, y z)= \\
& =r_{1} f_{n+1}^{t}(\ldots, y z)+\sum_{\substack{p \geq 2, i_{1}, \ldots, i_{p-1} \geq 1, i_{p} \geq 0 \\
i_{1}+\cdots+i_{p}=n}} \sum_{\sigma \in \operatorname{Sh}\left(i_{1}, \ldots, i_{p}\right)} \pm_{K} \frac{1}{(p-1) !} r_{p}\left(f_{i_{1}}^{t}(\ldots), \ldots, f_{i_{p}+1}^{t}(\ldots, y z)\right)= \\
& =r_{1}(\xi(t))+r_{1}\left(\sum_{i=0}^{n} \sum_{\sigma \in \operatorname{Sh}(i, n-i)} \pm_{K} f_{i+1}^{t}(\ldots, y) f_{n-i+1}^{t}(\ldots, z)\right)+ \\
& \sum_{\substack{p \geq 2, i_{1}, \ldots, i_{p-1} \geq 1, i_{p}, i_{p+1} \geq 0 \\
i_{1}+\cdots+i_{p+1}=n}} \sum_{\sigma \in \operatorname{Sh}\left(i_{1}, \ldots, i_{p+1}\right)} \pm_{K} \frac{1}{(p-1) !} r_{p}\left(f_{i_{1}}^{t}(\ldots), \ldots, f_{i_{p}+1}^{t}(\ldots, y) f_{i_{p+1}+1}^{t}(\ldots, z)\right)= \\
& =r_{1}(\xi(t))+\sum_{\substack{p, i_{1}, \ldots, i_{p-1} \geq 1, i_{p}, i_{p+1} \geq 0 \\
i_{1}+\cdots+i_{p+1}=n}} \sum_{\sigma \in \operatorname{Sh}\left(i_{1}, \ldots, i_{p+1}\right)} \pm_{K} \frac{1}{(p-1) !} r_{p}\left(f_{i_{1}}^{t}(\ldots), \ldots, f_{i_{p}+1}^{t}(\ldots, y)\right) f_{i_{p+1}+1}^{t}(\ldots, z)+ \\
& \pm_{K} \frac{1}{(p-1) !} f_{i_{p}+1}^{t}(\ldots, y) r_{p}\left(f_{i_{1}}^{t}(\ldots), \ldots, f_{i_{p+1}+1}^{t}(\ldots, z)\right)= \\
& =r_{1}(\xi(t))+\sum_{i=0}^{n} \sum_{\sigma \operatorname{Sh}(i, n-i)} \pm_{K} \frac{d}{d t} f_{i+1}^{t}(\ldots, y) f_{n-i+1}^{t}(\ldots, z) \pm_{K} f_{i+1}^{t}(\ldots, y) \frac{d}{d t} f_{n-i+1}^{t}(\ldots, z)= \\
& =r_{1}(\xi(t))+\frac{d}{d t}\left(\sum_{i=0}^{n} \sum_{\sigma \in \operatorname{Sh}(i, n-i)} \pm_{K} f_{i+1}^{t}(\ldots, y) f_{n-i+1}^{t}(\ldots, z)\right) .
\end{aligned}
$$

To sum up, we found that

$$
\xi^{\prime}(t)=r_{1}(\xi(t))
$$

thus

$$
\xi^{(n)}(t)=r_{1}^{n}(\xi(t)), \quad \forall n \geq 0
$$

Expanding in formal Taylor series,

$$
\xi(t)=\sum_{n \geq 0} \frac{t^{n}}{n !} \xi^{(n)}(0)=\sum_{n \geq 0} \frac{t^{n}}{n !} r_{1}^{n}(\xi(0))=\sum_{n \geq 0} \frac{t^{n}}{n !} r_{1}^{n}(0)=0 .
$$

2.3. Homotopy transfer for derived Poisson algebras. In this section, we prove a homotopy transfer theorem for derived Poisson algebras.

We start by recalling the standard Perturbation Lemma. To the best of our knowledge, this result first appeared implicitly in the paper [47], and explicitly in [7, 16]: for the treatment given here, see also [17, 20, 41].

Definition 2.9 ([13]). Let $\left(A, d_{A}\right)$ and $\left(B, d_{B}\right)$ be cochain complexes. A contraction $(\sigma, \tau, h)$ of $\left(A, d_{A}\right)$ onto $\left(B, d_{B}\right)$ is the datum of cochain maps $\sigma:\left(A, d_{A}\right) \rightarrow\left(B, d_{B}\right), \tau:\left(B, d_{B}\right) \rightarrow\left(A, d_{A}\right)$ and a contracting homotopy $h: A \rightarrow$ A such that

$$
\sigma \tau=\mathrm{id}_{B}, \quad h d_{A}+d_{A} h=\tau \sigma-\mathrm{id}_{A},
$$

and furthermore

$$
\sigma h=0, \quad h \tau=0, \quad h^{2}=0 .
$$


We denote such a contraction data by

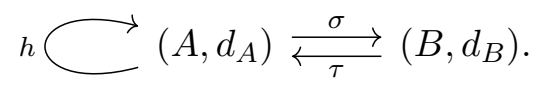

Lemma 2.10 ([47, 7, 16]). Let $(\sigma, \tau, h)$ be a contraction of $\left(A, d_{A}\right)$ onto $\left(B, d_{B}\right)$, and let $\delta_{A}: A \rightarrow A$ be a perturbation of the differential $d_{A}$ : that is, $\delta_{A}$ is a degree $(+1)$ map such that $\breve{d}_{A}:=d_{A}+\delta_{A}$ squares to zero. Then

$$
\delta_{B}:=\sum_{i=0}^{\infty} \sigma \delta_{A}\left(h \delta_{A}\right)^{i} \tau
$$

is a perturbation of the differential $d_{B}$, and $(\breve{\sigma}, \breve{\tau}, \breve{h})$ defined by

$$
\begin{aligned}
\breve{\sigma} & :=\sum_{i=0}^{+\infty} \sigma\left(\delta_{A} h\right)^{i}, \\
\breve{\tau} & :=\sum_{i=0}^{+\infty}\left(h \delta_{A}\right)^{i} \tau, \\
\breve{h} & :=\sum_{i=0}^{+\infty} h\left(\delta_{A} h\right)^{i}
\end{aligned}
$$

is a contraction of $\left(A, \breve{d}_{A}\right)$ onto $\left(B, \breve{d}_{B}:=d_{B}+\delta_{B}\right)$.

To be more precise, we should add some technical assumption ensuring convergence of the above infinite sums, but we will be loose in that respect and proceed formally.

Definition 2.11. Given dg commutative algebras $\left(A, d_{A}, m_{A}\right),\left(B, d_{B}, m_{B}\right)$ and a contraction $(\sigma, \tau, h)$ of $\left(A, d_{A}\right)$ onto $\left(B, d_{B}\right)$, we say that $(\sigma, \tau, h)$ is a semifull algebra contraction if the following identities are satisfied for all $a, b \in A$ and $x, y \in B$ :

$$
\begin{aligned}
h\left((-1)^{|a|+1} h(a) b+a h(b)\right) & =h(a) h(b), \\
h(a \tau(x)) & =h(a) \tau(x), \\
\sigma\left((-1)^{|a|+1} h(a) b+a h(b)\right) & =0, \\
\sigma(a \tau(x)) & =\sigma(a) x, \\
\tau(x y) & =\tau(x) \tau(y) .
\end{aligned}
$$

Remark 2.12. This class of contractions was introduced by Real [41]. More precisely, in [41, Definition 4.5], Equations (3)-(6) are replaced by the seemingly weaker

$$
\begin{aligned}
& h(h(a) h(b))=h(h(a) \tau(x))=0, \\
& \sigma(h(a) h(b))=\sigma(h(a) \tau(x))=0,
\end{aligned}
$$

whereas Equation (7) is maintained. It is straightforward that, if Equations (3)-(6) are satisfied, then the above equations are satisfied as well. In fact, our definition and the one from [41] are equivalent (in practice, the above equations are usually easier to check, on the other hand, Equations (3)-(6) will be the key to the following computations). To illustrate this fact, we show how to deduce Equation (3) from the above relations: these imply

$$
h(h(a) b)=h\left(h(a)\left(\tau \sigma-h d_{A}-d_{A} h\right)(b)\right)=-h\left(h(a) d_{A} h(b)\right),
$$

and similarly $h(a h(b))=-h\left(d_{A} h(a) h(b)\right)$. Thus

$$
h\left((-1)^{|a|+1} h(a) b+a h(b)\right)=-h d_{A}(h(a) h(b))=\left(d_{A} h-\tau \sigma+\mathrm{id}\right)(h(a) h(b))=h(a) h(b) .
$$

Equations (4)-(6) can be deduced similarly. 
Definition 2.13. Given a dg algebra $\left(A, d_{A}, m_{A}\right)$, an algebra perturbation of $d_{A}$ is a perturbation in the usual sense, which is furthermore an algebra derivation.

Proposition 2.14. Given dg algebras $\left(A, d_{A}, m_{A}\right),\left(B, d_{B}, m_{B}\right)$, a semifull algebra contraction $(\sigma, \tau, h)$ of $\left(A, d_{A}, m_{A}\right)$ onto $\left(B, d_{B}, m_{B}\right)$ and an algebra perturbation $\delta_{A}: A \rightarrow A$ of $d_{A}$, we apply the Perturbation Lemma 2.10. Then $\delta_{B}$ is an algebra perturbation of $d_{B}$, and $(\breve{\sigma}, \breve{\tau}, \breve{h})$ is a semifull algebra contraction of $\left(A, \breve{d}_{A}, m_{A}\right)$ onto $\left(B, \breve{d}_{B}, m_{B}\right)$.

Proof. This was proved in [41], see also [17, 20] for some related results. For completeness, we sketch a proof of this fact. To show that

we prove inductively that

$$
\breve{\tau}(x y)=\breve{\tau}(x) \breve{\tau}(y)
$$

$$
\left(h \delta_{A}\right)^{i} \tau(x y)=\sum_{j=0}^{i}\left(h \delta_{A}\right)^{j} \tau(x)\left(h \delta_{A}\right)^{i-j} \tau(y), \quad \forall i \geq 0 .
$$

The basis of the induction is Equation (7). Assume the above identity holds for a given $i$. Then

$$
\begin{gathered}
\left(h \delta_{A}\right)^{i+1} \tau(x y)=h \delta_{A}\left(\sum_{j=0}^{i}\left(h \delta_{A}\right)^{j} \tau(x)\left(h \delta_{A}\right)^{i-j} \tau(y)\right)= \\
=h\left(\delta_{A}\left(h \delta_{A}\right)^{i} \tau(x) \tau(y)\right)+ \\
+\sum_{j=1}^{i} h\left((-1)^{|x|} h \delta_{A}\left(h \delta_{A}\right)^{j-1} \tau(x) \delta_{A}\left(h \delta_{A}\right)^{i-j} \tau(y)+\delta_{A}\left(h \delta_{A}\right)^{j-1} \tau(x) h \delta_{A}\left(h \delta_{A}\right)^{i-j} \tau(y)\right)+ \\
+(-1)^{|x|} h\left(\tau(x) \delta_{A}\left(h \delta_{A}\right)^{i} \tau(y)\right)= \\
=\left(h \delta_{A}\right)^{i+1} \tau(x) \tau(y)+\sum_{j=1}^{i}\left(h \delta_{A}\right)^{j} \tau(x)\left(h \delta_{A}\right)^{i+1-j} \tau(y)+\tau(x)\left(h \delta_{A}\right)^{i+1} \tau(y),
\end{gathered}
$$

using Equations (3)-(4), which proves the inductive step.

Replacing the leftmost $h$ by $\sigma$ in the above computation, and using Equations (5)-(6) in the last passage, we see that

$$
\sigma \delta_{A}\left(h \delta_{A}\right)^{i} \tau(x y)=\sigma \delta_{A}\left(h \delta_{A}\right)^{i} \tau(x) y+(-1)^{|x|} x \sigma \delta_{A}\left(h \delta_{A}\right)^{i} \tau(y),
$$

which implies that $\delta_{B}$ is indeed an algebra perturbation.

Finally, to show that $(\breve{\tau}, \breve{\sigma}, \breve{h})$ satisfies Equations (3)-(6) in Definition 2.11, it suffices to show that it satisfies the equivalent conditions in Remark 2.12, which follow easily from the definitions.

The main result of this section is the following theorem, which says that we can transfer derived Poisson algebra structures along semifull algebra contractions.

Theorem 2.15. Let $\left(A, d_{A}, m_{A}\right)$ and $\left(B, d_{B}, m_{B}\right)$ be dg commutative algebras, and let $(\sigma, \tau, h)$ be a semifull algebra contraction of $\left(A, d_{A}, m_{A}\right)$ onto $\left(B, d_{B}, m_{B}\right)$. Let $\lambda_{n}: A^{\otimes n} \rightarrow A, n \geq 2$, be a family of maps making $\left(A, d_{A}=: \lambda_{1}, \lambda_{2}, \ldots, \lambda_{n}, \ldots\right)$ into a degree $k$ derived Poisson algebra. Via homotopy transfer along the contraction $(\sigma, \tau, h)$, there is an induced $L_{\infty}$ algebra structure on $B[-k]$, whose structure maps we denote by $\ell_{n}: B^{\otimes n} \rightarrow B, n \geq 2$. These maps make $\left(B, d_{B}=: \ell_{1}, \ell_{2}, \ldots, \ell_{n}, \ldots\right)$ into a degree $k$ derived Poisson algebra. Moreover, the $L_{\infty}$ quasi-isomorphism $\tau_{\infty}=\left(\tau_{1}, \tau_{2}, \ldots, \tau_{n}, \ldots\right)$ from $B[-k]$ to $A[-k]$ induced via homotopy transfer is a morphism of degree $k$ derived Poisson algebras.

Remark 2.16. It is a well known fact that $L_{\infty}$ algebra structures can be transferred along contractions. For a proof of this fact, we refer to [21]. See also $[1,14,5]$. The homotopy transfer theorem for $L_{\infty}$ algebra structures is a direct consequence of the Goldman-Millson theorem [11, 12]. 
Proof. In the following computations, to improve readability, we shall omit to make signs explicit, and instead denote by $\pm_{K}$ the appropriate Koszul signs (which can be worked out explicitly as explained in the previous subsections).

By homotopy transfer formulas, $\tau_{1}=\tau$, and $\tau_{n+1}, \ell_{n+1}, n \geq 1$, are defined recursively by

$$
\begin{aligned}
& \quad \tau_{n+1}\left(x_{1}, \ldots, x_{n}, y\right)= \\
& \qquad \sum_{\substack{p \geq 2, i_{1}, \ldots, i_{p-1} \geq 1, i_{p} \geq 0 \\
i_{1}+\cdots+i_{p}=n}} \sum_{\sigma \in \operatorname{Sh}\left(i_{1}, \ldots, i_{p}\right)} \pm_{K} \frac{1}{(p-1) !} h \lambda_{p}\left(\tau_{i_{1}}\left(x_{\sigma(1)}, \ldots, x_{\sigma\left(i_{1}\right)}\right), \ldots, \tau_{i_{p}+1}\left(x_{\sigma\left(n-i_{p}+1\right)}, \ldots, x_{\sigma(n)}, y\right)\right) \\
& \text { and } \sum_{\ell_{n+1}\left(x_{1}, \ldots, x_{n}, y\right)=} \sum_{K=2, i_{1}, \ldots, i_{p-1} \geq 1, i_{p} \geq 0} \pm_{\sigma \in \operatorname{Sh}\left(i_{1}, \ldots, i_{p}\right)} \frac{1}{(p-1) !} \sigma \lambda_{p}\left(\tau_{i_{1}}\left(x_{\sigma(1)}, \ldots, x_{\sigma\left(i_{1}\right)}\right), \ldots, \tau_{i_{p}+1}\left(x_{\sigma\left(n-i_{p}+1\right)}, \ldots, x_{\sigma(n)}, y\right)\right) .
\end{aligned}
$$

For notational simplicity in the following computations, we will omit the $x_{1}, \ldots, x_{n}$ variables, and abbreviate the above equations as

$$
\tau_{n+1}\left(x_{1}, \ldots, x_{n}, y\right)=\sum_{\substack{p \geq 2, i_{1}, \ldots, i_{p-1} \geq 1, i_{p} \geq 0 \\ i_{1}+\cdots+i_{p}=n}} \sum_{\sigma \in \operatorname{Sh}\left(i_{1}, \ldots, i_{p}\right)} \pm_{K} \frac{1}{(p-1) !} h \lambda_{p}\left(\tau_{i_{1}}(\ldots), \ldots, \tau_{i_{p}+1}(\ldots, y)\right)
$$

and

$$
\ell_{n+1}\left(x_{1}, \ldots, x_{n}, y\right)=\sum_{\substack{p \geq 2, i_{1}, \ldots, i_{p-1} \geq 1, i_{p} \geq 0 \\ i_{1}+\cdots+i_{p}=n}} \sum_{\sigma \in \operatorname{Sh}\left(i_{1}, \ldots, i_{p}\right)} \pm_{K} \frac{1}{(p-1) !} \sigma \lambda_{p}\left(\tau_{i_{1}}(\ldots), \ldots, \tau_{i_{p}+1}(\ldots, y)\right) .
$$

We shall prove first that $\tau_{\infty}$ satisfies the required compatibilities with the products. By assumption $\tau_{1}=\tau$ is a morphism of graded algebras. Proceeding inductively,

$$
\begin{aligned}
& \tau_{n+1}\left(x_{1}, \ldots, x_{n}, y z\right)= \\
& \sum_{\substack{p \geq 2, i_{1}, \ldots, i_{p-1} \geq 1, i_{p} \geq 0 \\
i_{1}+\cdots+i_{p}=n}} \sum_{\sigma \in \operatorname{Sh}\left(i_{1}, \ldots, i_{p}\right)} \pm_{K} \frac{1}{(p-1) !} h \lambda_{p}\left(\tau_{i_{1}}(\ldots), \ldots, \tau_{i_{p}+1}(\ldots, y z)\right)= \\
& \sum_{\substack{p \geq 2, i_{1}, \ldots, i_{p-1} \geq 1, i_{p}, i_{p+1} \geq 0 \\
i_{1}+\cdots+i_{p+1}=n}} \sum_{\sigma \in \operatorname{Sh}\left(i_{1}, \ldots, i_{p+1}\right)} \pm_{K} \frac{1}{(p-1) !} h \lambda_{p}\left(\tau_{i_{1}}(\ldots), \ldots, \tau_{i_{p}+1}(\ldots, y) \tau_{i_{p+1}+1}(\ldots, z)\right)= \\
& \sum_{\substack{p \geq 2, i_{1}, \ldots, i_{p-1} \geq 1, i_{p}, i_{p+1} \geq 0 \\
i_{1}+\cdots+i_{p+1}=n}} \sum_{\sigma \in \operatorname{Sh}\left(i_{1}, \ldots, i_{p+1}\right)} \pm_{K} \frac{1}{(p-1) !} h\left(\lambda_{p}\left(\tau_{i_{1}}(\ldots), \ldots, \tau_{i_{p}+1}(\ldots, y)\right) \tau_{i_{p+1}+1}(\ldots, z)\right)+ \\
& \pm_{K} \frac{1}{(p-1) !} h\left(\tau_{i_{p}+1}(\ldots, y) \lambda_{p}\left(\tau_{i_{1}}(\ldots), \ldots, \tau_{i_{p+1}+1}(\ldots, z)\right)\right)= \\
& \tau_{n+1}\left(x_{1}, \ldots, x_{n}, y\right) \tau_{1}(z) \pm_{K} \tau_{1}(y) \tau_{n+1}\left(x_{1}, \ldots, x_{n}, z\right)+\sum_{\substack{i, j \geq 1 \\
i+j=n}} \sum_{\substack{p \geq 2, i_{1}, \ldots, i_{p-1} \geq 1, i_{p} \geq 0 \\
i_{1}+\cdots+i_{p}=i}} \sum_{\substack{q \geq 2, j_{1}, \ldots, j_{q-1} \geq 1, j_{q} \geq 0 \\
j_{1}+\cdots+j_{q}=j}} \\
& \sum_{\sigma \in \operatorname{Sh}\left(i_{1}, \ldots, j_{q}\right)} \pm_{K} \frac{1}{(p-1) !} h\left(\lambda_{p}\left(\tau_{i_{1}}(\ldots), \ldots, \tau_{i_{p}+1}(\ldots, y)\right) \frac{1}{(q-1) !} h \lambda_{q}\left(\tau_{j_{1}}(\ldots), \ldots, \tau_{j_{q}+1}(\ldots, z)\right)\right)
\end{aligned}
$$




$$
\begin{aligned}
& \pm_{K} \frac{1}{(q-1) !} h\left(\frac{1}{(p-1) !} h \lambda_{p}\left(\tau_{i_{1}}(\ldots), \ldots, \tau_{i_{p}+1}(\ldots, y)\right) \lambda_{q}\left(\tau_{j_{1}}(\ldots), \ldots, \tau_{j_{q}+1}(\ldots, z)\right)\right)= \\
& \tau_{n+1}\left(x_{1}, \ldots, x_{n}, y\right) \tau_{1}(z) \pm_{K} \tau_{1}(y) \tau_{n+1}\left(x_{1}, \ldots, x_{n}, z\right)+\sum_{\substack{i, j \geq 1 \\
i+j=n}} \sum_{\substack{p \geq 2, i_{1}, \ldots, i_{p-1} \geq 1, i_{p} \geq 0 \\
i_{1}+\cdots+i_{p}=2, j_{1}, \ldots, j_{q-1} \geq 1, j_{q} \geq 0 \\
j_{1}+\cdots+j_{q}=j}} \\
& \quad \sum_{\sigma \in \operatorname{Sh}\left(i_{1}, \ldots, j_{q}\right)} \pm_{K} \frac{1}{(p-1) !} h \lambda_{p}\left(\tau_{i_{1}}(\ldots), \ldots, \tau_{i_{p}+1}(\ldots, y)\right) \frac{1}{(q-1) !} h \lambda_{q}\left(\tau_{j_{1}}(\ldots), \ldots, \tau_{j_{q}+1}(\ldots, z)\right)= \\
& \sum_{i=0}^{n} \sum_{\sigma \in \operatorname{Sh}(i, n-i)} \pm_{K} \tau_{i+1}(\ldots, y) \tau_{n-i+1}(\ldots, z),
\end{aligned}
$$

where we used the identities (3)-(4).

Comparing the formula for $\tau_{n+1}$ and the one for $\ell_{n+1}$ at the beginning of the proof, to show that the $\ell_{n+1}$ are multi-derivations we can follow the above computations, replacing the leftmost $h$ by $\sigma$ and using the identities (5)-(6) (instead of (3)-(4)) when appropriate.

\section{Shifted DeRIVEd Poisson MANifolds}

\subsection{Shifted derived Poisson manifolds.}

Definition 3.1. $A(-k)$-shifted derived Poisson manifold is a $\mathbb{Z}$-graded manifold $\mathcal{M}$ whose sheaf of functions $C_{\mathcal{M}}^{\infty}$ is a sheaf of degree $k$ derived Poisson algebras.

Equivalently, a $(-k)$-shifted derived Poisson manifold is a $\mathbb{Z}$-graded manifold $\mathcal{M}$ such that $C^{\infty}(\mathcal{M})$, the space of global functions on $\mathcal{M}$, is endowed with a degree $k$ derived Poisson algebra structure

$$
\lambda_{l}:\left(C^{\infty}(\mathcal{M})\right)^{\otimes l} \rightarrow C^{\infty}(\mathcal{M}), \quad l \geq 1 .
$$

Example 3.2. Let $\mathfrak{g}$ be a finite dimensional $L_{\infty}$ algebra. By extending the $L_{\infty}$ structure maps $\lambda_{l}(l \geq 1)$ on $\mathfrak{g}$ to the completed symmetric algebra $\hat{S}(\mathfrak{g}[k])$ via the Leibniz rule, one obtains a degree $k$ derived Poisson algebra on $\hat{S}(\mathfrak{g}[k])=C^{\infty}\left(\mathfrak{g}^{\vee}[-k]\right)$. Thus $\mathfrak{g}^{\vee}[-k]$ is a $(-k)$-shifted derived Poisson manifold, called $(-k)$ shifted derived Lie Poisson manifold. In particular, $\mathfrak{g}^{\vee}$ is a derived Lie Poisson manifold. See [29].

If $\mathfrak{g}$ is an ordinary Lie algebra, $(\mathfrak{g}[0])^{\vee}=\mathfrak{g}^{\vee}$ admits a Poisson manifold structure, called Lie-Poisson structure. On the other hand, we have the standard Schouten algebra $\Lambda^{\bullet} \mathfrak{g}$, which corresponds to the (-1)-shifted Poisson manifold structure on $\mathfrak{g}^{\vee}[-1]$.

The following proposition relates the notion of shifted derived Poisson manifolds introduced above with the one defined by Pridham $[39,40]$.

Let $\mathcal{M}$ be a $\mathbb{Z}$-graded manifold. By $\hat{\mathscr{X}}_{\text {poly }}^{\bullet}(\mathcal{M}, n)$, we denote the formally completed Schouten-Nijenhuis algebra of $n$-shifted polyvector fields on $\mathcal{M}$ (see Appendix C).

Proposition 3.3. $A(-k)$-shifted derived Poisson manifold is equivalent to a dg manifold $(\mathcal{M}, Q)$ equipped with a formal series of $(k-2)$-shifted polyvector fields $\pi=\sum_{l=2}^{\infty} \pi_{l}$ satisfying the Maurer-Cartan equation:

$$
[Q, \pi]+\frac{1}{2}[\pi, \pi]=0
$$

where $\pi_{l} \in \hat{\mathscr{X}}_{\text {poly }}^{l}(\mathcal{M}, k-2)$ is of total degree $(+1)$ in $\hat{\mathscr{X}}_{\text {poly }}(\mathcal{M}, k-2)[k-1]$.

Proof. Assume that $\mathcal{M}$ is a $(-k)$-shifted derived Poisson manifold. Let $\lambda_{l}:\left(C^{\infty}(\mathcal{M})\right)^{\otimes l} \rightarrow C^{\infty}(\mathcal{M})$, $l \geq 1$, be the multi-brackets of the corresponding shifted $L_{\infty}$ algebra. 
The unary bracket $\lambda_{1}: C^{\infty}(\mathcal{M}) \rightarrow C^{\infty}(\mathcal{M})$ is a derivation of degree $(+1)$ and squares to zero. Thus it determines a homological vector field $Q$ on $\mathcal{M}$, which is also denoted by $\pi_{1}$. Since $\left(\lambda_{l}\right)_{l=1}^{\infty}$ defines an $L_{\infty}$ algebra structure on $C^{\infty}(\mathcal{M})[-k]$, for any $l \geq 2, \lambda_{l}$ is a skew-symmetric multi-bracket on $C^{\infty}(\mathcal{M})[-k]$, i.e.,

$$
\lambda_{l}(\cdots, f, g \cdots)=-(-1)^{|f|^{[k]}|g|^{[k]}} \lambda_{l}(\cdots, g, f \cdots) .
$$

Let $\pi_{l}\left(f_{1}, f_{2}, \cdots, f_{l}\right)=(-1)^{\star} \lambda_{l}\left(f_{1}, f_{2}, \cdots, f_{l}\right)$, where $\star=(l-1)\left|f_{1}\right|^{[k]}+(l-2)\left|f_{2}\right|^{[k]}+\cdots+\left|f_{l-1}\right|^{[k]}$, then $\pi_{l}$ is a symmetric multilinear map on $C^{\infty}(\mathcal{M})[-k+1]$, i.e.

$$
\pi_{l}(\cdots, f, g \cdots)=(-1)^{|f|^{[k-1]}|g|^{[k-1]}} \pi_{l}(\cdots, g, f \cdots) .
$$

It is clear that $\pi_{l}$ is a multi-derivation in each argument. According to Appendix C, $\pi_{l}$ can be considered as a $(k-2)$-shifted $l$-polyvector field on $\mathcal{M}$, i.e. $\pi_{l} \in \mathscr{X}_{\text {poly }}^{l}(\mathcal{M}, k-2)$. Moreover, its total degree is

$$
\left\|\pi_{l}\right\|_{k-2}=\left|\pi_{l}\right|-l(k-1)=\left|\lambda_{l}\right|-l(k-1)=2-k .
$$

Therefore, when being considered as an element in $\mathscr{X}_{\text {poly }}^{l}(\mathcal{M}, k-2)[k-1], \pi_{l}$ is of degree $(+1)$. Let $\Lambda=Q+\pi=\sum_{l \geq 1} \pi_{l}$. Then $\Lambda$ is of total degree $(+1)$. Let

$$
\Pi=\frac{1}{2}[\Lambda, \Lambda]
$$

According to Lemma C.3,

$$
\Pi=\Lambda \circ \Lambda=\sum_{m, n \geq 1} \pi_{m} \circ \pi_{n} \in \bigoplus_{l=2}^{\infty} \mathscr{X}_{\text {poly }}^{l}(\mathcal{M}, k-2)[k-1] .
$$

The weight $p$ component of $\Pi$ is

$$
\Pi_{p}=\sum_{m+n-1=p} \pi_{m} \circ \pi_{n}
$$

For any $f_{1}, f_{2}, \cdots, f_{m+n-1} \in C^{\infty}(\mathcal{M})$,

$$
\begin{aligned}
& \left.\sum_{\sigma \in \operatorname{Sh}(m, n-1)} \epsilon_{m} \circ \pi_{n}\right)\left(f_{1}, \cdots, f_{m+n-1}\right) \\
= & \sum_{\sigma \in \operatorname{Sh}(m, n-1)}(-1)^{\star} \\
& \epsilon^{[k-1]}(\sigma) \pi_{m}\left(\pi_{n}\left(f_{\sigma(1)}, \cdots, f_{\sigma(n)}\right), f_{\sigma(n+1)}, \cdots, f_{\sigma(m+n-1)}\right) \\
& \text { where } \star=(n-1)\left|f_{\sigma(1)}\right|^{[k]}+\cdots+\left|f_{\sigma(n-1)}\right|^{[k]}+(m-1)\left(\left|f_{\sigma(1)}\right|^{[k]}+\cdots+\left|f_{\sigma(n)}\right|^{[k]}+2-n\right)+(m-2)\left|f_{\sigma(n+1)}\right|^{[k]}+\cdots+\left|f_{\sigma(m+n-2)}\right|^{[k]} \\
= & (-1)^{(m-1) n+K} \sum_{\sigma \in \operatorname{Sh}(m, n-1)}|\sigma| \epsilon^{[k]}(\sigma) \lambda_{m}\left(\lambda_{n}\left(f_{\sigma(1)}, \cdots, f_{\sigma(n)}\right), f_{\sigma(n+1)}, \cdots, f_{\sigma(m+n-1)}\right) .
\end{aligned}
$$

Here $K$ denotes the constant integer $(p-1)\left|f_{1}\right|^{[k]}+(p-2)\left|f_{2}\right|^{[k]}+\cdots+\left|f_{p-1}\right|^{[k]}$.

Equation (8) is equivalent to the condition that $\Pi_{p}=0$ for all $p \geq 2$. According to Equation (9), the latter is equivalent to that $\forall f_{1}, \cdots, f_{p} \in C^{\infty}(\mathcal{M})$,

$$
\sum_{m+n-1=p}(-1)^{(m-1) n} \sum_{\sigma \in \operatorname{Sh}(m, n-1)}|\sigma| \epsilon^{[k]}(\sigma) \lambda_{m}\left(\lambda_{n}\left(f_{\sigma(1)}, \cdots, f_{\sigma(n)}\right), f_{\sigma(n+1)}, \cdots, f_{\sigma(p)}\right)=0 .
$$

It is clear that this is exactly the generalized Jacobi identity of the $L_{\infty}$ algebra structure on $C^{\infty}(\mathcal{M})[-k]$. The converse is proved by going backwards. This concludes the proof. 
Example 3.4. $A(-1)$-shifted derived Poisson manifold is equivalent to a dg manifold $(\mathcal{M}, Q)$ equipped with a formal series $\pi=\sum_{l=2}^{\infty} \pi_{l}$ satisfying the Maurer-Cartan equation:

$$
[Q, \pi]+\frac{1}{2}[\pi, \pi]=0
$$

where, for each $l \geq 2, \pi_{l} \in \Gamma\left(S^{l} T_{\mathcal{M}}\right)$ is of degree $(+1)$, and the bracket in Equation (10) is the canonical Poisson bracket on $\Gamma\left(\hat{S} T_{\mathcal{M}}\right)$ being identified with the space of formal polynomials on the symplectic manifold $T_{\mathcal{M}}^{\vee}$

Definition 3.5. Let $\mathcal{M}$ and $\mathcal{M}^{\prime}$ be $(-k)$-shifted derived Poisson manifolds with structure maps $\lambda_{l}:\left(C^{\infty}(\mathcal{M})\right)^{\otimes l}$ $\rightarrow C^{\infty}(\mathcal{M})$ and $\lambda_{l}^{\prime}:\left(C^{\infty}\left(\mathcal{M}^{\prime}\right)\right)^{\otimes l} \rightarrow C^{\infty}\left(\mathcal{M}^{\prime}\right), l \geq 1$, respectively. A morphism of $(-k)$-shifted derived Poisson manifolds from $\mathcal{M}$ to $\mathcal{M}^{\prime}$ is a map of $\mathbb{Z}$-graded manifolds $\phi: \mathcal{M} \rightarrow \mathcal{M}^{\prime}$ together with a collection of maps

$$
\varphi_{n}:\left(C^{\infty}\left(\mathcal{M}^{\prime}\right)\right)^{\otimes n} \rightarrow C^{\infty}(\mathcal{M}), \quad n=2,3, \cdots
$$

such that $\varphi_{\infty}=\left(\varphi_{1}=\phi^{*}, \varphi_{2}, \varphi_{3}, \cdots\right)$ is a morphism of degree $k$ derived Poisson algebras from $\left(C^{\infty}\left(\mathcal{M}^{\prime}\right)\right.$, $\left.\lambda_{1}^{\prime}, \lambda_{2}^{\prime}, \cdots, \lambda_{n}^{\prime}, \cdots\right)$ to $\left(C^{\infty}(\mathcal{M}), \lambda_{1}, \lambda_{2}, \cdots, \lambda_{n}, \cdots\right)$.

In particular, $\phi: \mathcal{M} \rightarrow \mathcal{M}^{\prime}$ is a map of dg manifolds.

3.2. $L_{\infty}$ algebroids and shifted derived Poisson manifolds. Below we follow Bruce [8] for the notations, who considered the $\mathbb{Z}_{2}$-case.

Definition 3.6. An $L_{\infty}$ algebroid consists of a vector bundle $\mathcal{L} \rightarrow \mathcal{M}$ of $\mathbb{Z}$-graded manifolds together with

- a sequence of multilinear maps $\lambda_{l}: \Lambda^{l} \Gamma(\mathcal{L}) \rightarrow \Gamma(\mathcal{L})$ of degree $(2-l), l \geq 1$, called multi-brackets, that determine an $L_{\infty}$ algebra structure on $\Gamma(\mathcal{L})$, the space of sections of $\mathcal{L} \rightarrow \mathcal{M}$; and

- a sequence of bundle maps $\rho_{l}: \Lambda^{l} \mathcal{L} \rightarrow T_{\mathcal{M}}$ of degree $(1-l), l \geq 0$, called multi-anchor maps, that induce a morphism of $L_{\infty}$ algebras from $\Gamma(\mathcal{L})$ to $\mathscr{X}(\mathcal{M})$

such that the following compatibility condition is satisfied:

$$
\begin{aligned}
\lambda_{l}\left(a_{1}, a_{2}, \cdots, a_{l-1}, f a_{l}\right)= & \rho_{l-1}\left(a_{1}, a_{2}, \cdots,\right. \\
& \left.a_{l-1}\right)(f) a_{l} \\
& +(-1)^{\left(l+\left|a_{1}\right|+\cdots+\left|a_{l-1}\right|\right)|f|} f \lambda_{l}\left(a_{1}, a_{2}, \cdots, a_{l-1}, a_{l}\right)
\end{aligned}
$$

$\forall l \geq 1, a_{1}, \cdots, a_{l} \in \Gamma(\mathcal{L})$, and $f \in C^{\infty}(\mathcal{M})$.

Remark 3.7. Note that the image of $\rho_{1}$ may not be integrable. When $\mathcal{M}$ is an ordinary smooth manifold $M$ (being considered of degree zero) and the vector bundle $L=\bigoplus_{i>0} L^{i} \rightarrow M$ is a non-negative graded vector bundle, due to degree reasons, all higher anchor maps $\rho_{l}$ vanish except for $\rho_{1}$, which must be a bundle map $L^{0} \rightarrow T_{M}$. Our notion of $L_{\infty}$ algebroids reduces to the one studied by Laurent-Gengoux et. al. [30]. In this case, $\rho_{1}\left(L^{0}\right)$ defines a singular foliation on $M$. When $L$ is concentrated in degree 0 , it becomes a usual Lie algebroid over $M$.

We also note that various forms of $L_{\infty}$ algebroids have appeared in the literature. We refer the reader to $[24,26,27,45,54,4,46,19]$ and the references there on the related topic.

The following proposition extends Theorem 1 and Corollary 2 in [8] to the $\mathbb{Z}$-graded context.

Proposition 3.8. Let $\mathcal{L} \rightarrow \mathcal{M}$ be a vector bundle of $\mathbb{Z}$-graded manifolds, and let $k \in \mathbb{Z}$ be a fixed integer. The following statements are equivalent.

(1) The vector bundle $\mathcal{L} \rightarrow \mathcal{M}$ is an $L_{\infty}$ algebroid.

(2) The space $\Gamma(\hat{S}(\mathcal{L}[k]))$ is a degree $k$ derived Poisson algebra, whose lth-bracket $\lambda_{l}: \Lambda^{l} \Gamma(\mathcal{L}) \rightarrow \Gamma(\mathcal{L})$ is of weight $(1-l)$. 
(3) The graded manifold $\mathcal{L}^{\vee}[-k]$ is a $(-k)$-shifted derived Poisson manifold, where the weight of the lth-bracket on $C^{\infty}\left(\mathcal{L}^{\vee}[-k]\right)$ is $(1-l)$.

Recall that elements in $\Gamma\left(S^{m}(\mathcal{L}[k])\right)$ are of weight $m$. The weight of the $l$ th-bracket

$$
\lambda_{l}: \Gamma\left(S^{\bullet}(\mathcal{L}[k])\right) \times \cdots \times \Gamma\left(S^{\bullet}(\mathcal{L}[k])\right) \rightarrow \Gamma\left(S^{\bullet}(\mathcal{L}[k])\right)
$$

is the difference of weights on both sides.

Proof. Note that (2) and (3) are clearly equivalent by definition. Below, we prove the equivalence between (1) and (2).

Assume that $\mathcal{L} \rightarrow \mathcal{M}$ is an $L_{\infty}$ algebroid. Then the $L_{\infty}$ algebra structure on $\Gamma(\mathcal{L})$ together with the multianchor maps defines a degree $k$ derived Poisson algebra, via the Leibniz rule, on $\Gamma(\hat{S}(\mathcal{L}[k]))$ by the following generating relations:

$$
\begin{aligned}
\lambda_{l}\left(a_{1}, \cdots, a_{l-1}, f\right) & =\rho_{l-1}\left(a_{1}, \cdots, a_{l-1}\right) f, \quad(l \geq 1) \\
\lambda_{l}(f, g, \cdots) & =0, \quad(l \geq 2),
\end{aligned}
$$

$\forall a_{1}, \cdots, a_{l} \in \Gamma(\mathcal{L}[k])$, and $f, g \in C^{\infty}(\mathcal{M})$. It is easy to check that its $l$ th bracket is of weight $(1-l)$.

The converse can be proved by going backwards using Equations (12)-(13).

Remark 3.9. We note that Vitagliano has already observed a similar result in the algebraic context of $L R_{\infty}$ algebras. For details, see [54, 53].

Let $\mathcal{L} \rightarrow \mathcal{M}$ be an $L_{\infty}$ algebroid with structure maps $\left(\lambda_{l}\right)_{l \geq 1}$ and $\left(\rho_{l}\right)_{l \geq 0}$ as in Definition 3.6. The unary bracket $\lambda_{1}: \Gamma(\mathcal{L}) \rightarrow \Gamma(\mathcal{L})$ and $\rho_{0} \in \mathscr{X}(\mathcal{M})$ are compatible:

$$
\lambda_{1}(f a)=\rho_{0}(f) a+(-1)^{|f|} f \lambda_{1}(a), \quad \forall a \in \Gamma(L), f \in C^{\infty}(\mathcal{M}) .
$$

Introduce a dual map $\lambda_{1}^{\vee}: \Gamma\left(\mathcal{L}^{\vee}\right) \rightarrow \Gamma\left(\mathcal{L}^{\vee}\right)$ by

$$
\left\langle\lambda_{1}^{\vee}(\xi), a\right\rangle=\rho_{0}\langle\xi, a\rangle-(-1)^{|\xi|}\left\langle\xi, \lambda_{1}(a)\right\rangle, \quad \forall \xi \in \Gamma\left(\mathcal{L}^{\vee}\right), \forall a \in \Gamma(L) .
$$

Since $\lambda_{1}^{2}=0$, it follows that $\left(\lambda_{1}^{\vee}\right)^{2}=0$. Hence $\left(\Gamma\left(\mathcal{L}^{\vee}\right), \lambda_{1}^{\vee}\right)$ is a cochain complex.

Definition 3.10. Let $\mathcal{L}_{1} \rightarrow \mathcal{M}_{1}$ and $\mathcal{L}_{2} \rightarrow \mathcal{M}_{2}$ be $L_{\infty}$ algebroids.

(1) A morphism of $L_{\infty}$ algebroids from $\mathcal{L}_{1} \rightarrow \mathcal{M}_{1}$ to $\mathcal{L}_{2} \rightarrow \mathcal{M}_{2}$ is a sequence of bundle maps

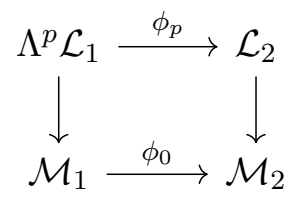

for $p=1,2, \cdots$, such that the induced map $\phi: \mathcal{L}_{1}[1] \rightarrow \mathcal{L}_{2}[1]$ is a map of dg manifolds. ${ }^{1}$

(2) A quasi-isomorphism from $\mathcal{L}_{1} \rightarrow \mathcal{M}_{1}$ to $\mathcal{L}_{2} \rightarrow \mathcal{M}_{2}$ is a morphism of $L_{\infty}$ algebroids $\phi: \mathcal{L}_{1} \rightarrow \mathcal{L}_{2}$ such that $\phi_{1}^{\vee}: \Gamma\left(\mathcal{L}_{2}^{\vee}\right) \rightarrow \Gamma\left(\mathcal{L}_{1}^{\vee}\right)$ is a quasi-isomorphism of cochain complexes.

The following fact can be easily verified.

\footnotetext{
${ }^{1}$ This means that the induced morphism of commutative algebras over $\phi_{0}^{\vee}: C^{\infty}\left(\mathcal{M}_{2}\right) \rightarrow C^{\infty}\left(\mathcal{M}_{1}\right)$ :

$$
\phi^{\vee}: C^{\infty}\left(\mathcal{L}_{2}[1]\right)=\Gamma\left(\hat{S}\left(\mathcal{L}_{2}^{\vee}[-1]\right)\right) \rightarrow C^{\infty}\left(\mathcal{L}_{1}[1]\right)=\Gamma\left(\hat{S}\left(\mathcal{L}_{1}^{\vee}[-1]\right)\right)
$$

commutes with the homological vector fields $Q_{1}$ and $Q_{2}: Q_{2} \circ \phi^{\vee}=\phi^{\vee} \circ Q_{1}$. Here $Q_{1}$ and $Q_{2}$ are, respectively, homological vector fields on $\mathcal{L}_{1}[1]$ and $\mathcal{L}_{2}[1]$ corresponding to the $L_{\infty}$ algebroid structures as in Proposition A.1.
} 
Proposition 3.11. Let $\mathcal{L}_{1} \rightarrow \mathcal{M}$ and $\mathcal{L}_{2} \rightarrow \mathcal{M}$ be $L_{\infty}$ algebroids. Let

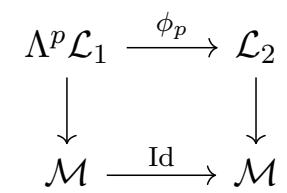

(for $p=1,2, \cdots)$ be a morphism of $L_{\infty}$ algebroids. Then there induces a morphism of $(-k)$-shifted derived Poisson manifolds from $\mathcal{L}_{2}^{\vee}[-k]$ to $\mathcal{L}_{1}^{\vee}[-k]$.

Proof. Consider the morphism of $L_{\infty}$ algebroids from $\mathcal{L}_{1} \rightarrow \mathcal{M}_{1}$ to $\mathcal{L}_{2} \rightarrow \mathcal{M}_{2}$ as in Definition 3.10. In general, $\phi_{0}: \mathcal{M}_{1} \rightarrow \mathcal{M}_{2}$ is a map of graded manifold. When $\mathcal{M}_{1}=\mathcal{M}_{2}=\mathcal{M}$ and $\phi_{0}=\mathrm{Id}$, there induces a family of $C^{\infty}(\mathcal{M})$-multilinear maps

$$
\tilde{\phi}_{p}: \Lambda^{p} \Gamma\left(\mathcal{L}_{1}\right) \rightarrow \Gamma\left(\mathcal{L}_{2}\right) .
$$

As $\left(\phi_{p}\right)_{p \geq 1}$ consists a morphism of $L_{\infty}$ algebroids from $\mathcal{L}_{1} \rightarrow \mathcal{M}$ to $\mathcal{L}_{2} \rightarrow \mathcal{M}$, the above $\left(\tilde{\phi}_{p}\right)_{p \geq 1}$ defines a morphism of $L_{\infty}$ algebras from $\Gamma\left(\mathcal{L}_{1}\right)$ to $\Gamma\left(\mathcal{L}_{2}\right)$. Moreover, each $\tilde{\phi}_{p}$ is skew-symmetric.

Then one extends $\left(\tilde{\phi}_{p}\right)_{p \geq 1}$ to a family of skew-symmetric maps:

$$
\tilde{\phi}_{p}: \Lambda^{p}\left(\Gamma\left(\hat{S}\left(\mathcal{L}_{1}[k]\right)\right)[-k]\right) \rightarrow \Gamma\left(\hat{S}\left(\mathcal{L}_{2}[k]\right)\right)[-k],
$$

satisfying Equation (2). Hence we obtain a morphism of degree $k$ derived Poisson algebras from $\Gamma\left(\hat{S}\left(\mathcal{L}_{1}[k]\right)\right)$ to $\Gamma\left(\hat{S}\left(\mathcal{L}_{2}[k]\right)\right)$, or equivalently, a morphism of $(-k)$-shifted derived Poisson manifolds from $\mathcal{L}_{2}^{\vee}[-k]$ to $\mathcal{L}_{1}^{\vee}[-k]$.

\section{The $(+1)$-Shifted derived Poisson Algebra ARISING From a LiE PAIR}

4.1. First construction: $L_{\infty}$ algebroid arising from a Lie pair. By a Lie pair $(L, A)$, we mean an ordinary (non-graded) Lie algebroid $\left(L,[\cdot, \cdot]_{L}, \rho_{L}\right)$ over an ordinary (non-graded) smooth manifold $M$, together with a Lie subalgebroid $\left(A,[\cdot, \cdot]_{A}, \rho_{A}\right)$ of $L$ over the same base $M$.

For convenience, let us denote the quotient vector bundle $L / A$ by $B$. Denote $\operatorname{pr}_{B}: L \rightarrow B$ the projection map. Note that $B$ is naturally an $A$-module:

$$
\nabla_{a}^{\mathrm{Bott}} b=\operatorname{pr}_{B}[a, l]_{L}
$$

where $a \in \Gamma(A), b \in \Gamma(B)$ and $l \in \Gamma(L)$ satisfying $\operatorname{pr}_{B}(l)=b$. The flat $A$-connection $\nabla^{\text {Bott }}$ on $B$ is also known as the Bott connection $[6,10]$.

Let

$$
\begin{aligned}
& \Omega_{A}^{\bullet}=\oplus_{k=0} \Gamma\left(\Lambda^{k} A^{\vee}\right)[-k], \\
& \Omega_{A}^{\bullet}\left(\Lambda^{\bullet} B\right)=\oplus_{k, l} \Gamma\left(\Lambda^{k} A^{\vee} \otimes \Lambda^{l} B\right)[-k+l] .
\end{aligned}
$$

Note that elements in $\Omega_{A}^{k}\left(\Lambda^{l} B\right)$ are of degree $k-l$. Denote by

$$
d_{A}^{\text {Bott }}: \quad \Omega_{A}^{k}\left(\Lambda^{l} B\right) \rightarrow \Omega_{A}^{k+1}\left(\Lambda^{l} B\right)
$$

the standard Chevalley-Eilenberg differential corresponding to the Bott $A$-connection on $\Lambda^{\bullet} B$.

Proposition 4.1. Let $(L, A)$ be a Lie pair. Any splitting $\mathrm{j}: B \rightarrow L$ of the exact sequence

$$
0 \rightarrow A \stackrel{\text { i }}{\rightarrow} L \stackrel{\mathrm{pr}_{B}}{\rightarrow} B \rightarrow 0
$$

induces an $L_{\infty}$ algebroid structure on the $\mathbb{Z}$-graded vector bundle $A[1] \oplus B \rightarrow A[1]$. 
Proof. A splitting of (15) is a pair of maps j: $B \rightarrow L$ and $\mathrm{pr}_{A}: L \rightarrow A$ such that $\mathrm{pr}_{B} \circ \mathrm{j}=\mathrm{id}_{B}, \mathrm{pr}_{A} \circ \mathrm{i}=\mathrm{id}_{A}$ and $\mathrm{i} \circ \mathrm{pr}_{A}+\mathrm{j} \circ \mathrm{pr}_{B}=\mathrm{id}_{L}$ :

$$
0 \rightleftarrows A \underset{\mathrm{pr}_{A}}{\stackrel{\mathrm{i}}{\rightleftarrows}} L \underset{\mathrm{j}}{\stackrel{\mathrm{pr}_{B}}{\rightleftarrows}} B \underset{\rightleftarrows}{\rightleftarrows}
$$

We therefore obtain an isomorphism of vector bundles over $M$ :

$$
\kappa: A \oplus B \stackrel{\simeq}{\longrightarrow} L, \quad(a, b) \mapsto \mathrm{i}(a)+\mathrm{j}(b),
$$

for all $a \in A$ and $b \in B$. Consider the $\mathbb{Z}$-graded vector bundle $\mathcal{L} \rightarrow A[1]$, where $\mathcal{L}:=A[1] \oplus B$. Note that the base of $\mathcal{L}$ is $A[1]$. Then $\mathcal{L}[1]=A[1] \oplus B[1] \cong L[1]$ according to (16).

Since $L$ is a Lie algebroid, the standard Chevalley-Eilenberg differential $d_{L}: \Gamma\left(\Lambda^{\bullet} L^{\vee}\right) \rightarrow \Gamma\left(\Lambda^{\bullet+1} L^{\vee}\right)$ defines a homological vector field $Q_{L}$ on $L[1]$. Via the identification (16), one obtains a homological vector field $Q$ on $\mathcal{L}[1]$. Since $A$ is a Lie subalgebroid of $L$, it is simple to see that the homological vector field $Q$ on $\mathcal{L}[1]$ is indeed tangent to the zero section $A[1] \subset \mathcal{L}[1]$, whose restriction can be identified with the Chevalley-Eilenberg differential $d_{A}$ on $A[1]$. Thus by Proposition A.1, $\mathcal{L}=A[1] \oplus B \rightarrow A[1]$ is indeed an $L_{\infty}$ algebroid.

Remark 4.2. According to Proposition 4.17, different choices of splittings give rise to isomorphic $L_{\infty}$ algebras $\Gamma(\mathcal{L}) \cong \Omega_{A}^{\bullet}(B)$, where the isomorphism is given by a collection of multilinear maps

$$
\varphi_{n}: \wedge^{n} \Omega_{A}^{\bullet}(B) \rightarrow \Omega_{A}^{\bullet}(B), \quad n=1,2, \cdots
$$

with $\varphi_{1}=\mathrm{id}$. However since $\varphi_{n}(n \geq 2)$ is not $C^{\infty}(A[1])=\Omega_{A^{\bullet}}$-multilinear, $\varphi_{n}$ does not correspond to a bundle map $\wedge^{n} \mathcal{L} \rightarrow \mathcal{L}$. Therefore, the corresponding $L_{\infty}$ algebroid structures on the $\mathbb{Z}$-graded vector bundle $\mathcal{L}=A[1] \oplus B \rightarrow A[1]$ are "not" isomorphic.

As an immediate consequence of Proposition 4.1, we have the following

Proposition 4.3. Let $(L, A)$ be a Lie pair. Then any splitting of (15) induces a degree $(+1)$ derived Poisson algebra structure on tot $\Omega_{A}^{\bullet}\left(\Lambda^{\bullet} B\right)$, where the multiplication is the wedge product, and the shifted $L_{\infty}$ brackets are given as follows:

(1) The unary bracket $l_{1}$ is the Chevalley-Eilenberg differential $d_{A}^{\text {Bott }}$ as in Equation (14).

(2) The binary bracket

$$
\{-,-\}_{2}: \Omega_{A}^{i}\left(\Lambda^{j} B\right) \times \Omega_{A}^{p}\left(\Lambda^{l} B\right) \rightarrow \Omega_{A}^{i+p}\left(\Lambda^{j+l-1} B\right)
$$

is generated by the following relations:

a) $\{u, v\}_{2}=\operatorname{pr}_{B}[u, v]_{L}, \quad \forall u, v \in \Gamma(B)$;

b) $\{u, \omega\}_{2}=\operatorname{pr}_{A^{\vee}}\left(L_{u} \omega\right), \quad \forall u \in \Gamma(B), \omega \in \Gamma\left(A^{\vee}\right)$;

c) $\{u, f\}_{2}=\rho_{L}(u) f, \quad \forall u \in \Gamma(B), f \in C^{\infty}(M)$;

d) $\left\{\omega_{1}, \omega_{2}\right\}_{2}=0, \quad \forall \omega_{1}, \omega_{2} \in \Omega_{A}^{\bullet}$.

(3) The ternary bracket

$$
\{-,-,-\}_{3}: \Omega_{A}^{i}\left(\Lambda^{j} B\right) \times \Omega_{A}^{p}\left(\Lambda^{l} B\right) \times \Omega_{A}^{r}\left(\Lambda^{s} B\right) \rightarrow \Omega_{A}^{i+p+r-1}\left(\Lambda^{j+l+s-2} B\right)
$$

is $C^{\infty}(M)$-linear in each entry and generated by the following relations:

a) $\{-,-,-\}_{3}$ vanishes when being restricted to $\Gamma(B) \times \Gamma(B) \times \Gamma(B), \Gamma(B) \times \Gamma\left(A^{\vee}\right) \times \Gamma\left(A^{\vee}\right)$, and $\Gamma\left(A^{\vee}\right) \times \Gamma\left(A^{\vee}\right) \times \Gamma\left(A^{\vee}\right)$;

b) $\{u, v, \omega\}_{3}=\left\langle\operatorname{pr}_{A}[u, v]_{L}, \omega\right\rangle$, for all $u, v \in \Gamma(B)$ and $\omega \in \Gamma\left(A^{\vee}\right)$.

(4) All the rest of higher brackets vanish.

Here we have used the identification $L \cong A \oplus B$ and $L^{\vee} \cong A^{\vee} \oplus B^{\vee}$. 
To prove Proposition 4.3, we need to give an explicit expression for the homological vector field $Q$ on $\mathcal{L}[1]$. Choosing a splitting of (15), we have an identification $L \cong A \oplus B$. Besides the Bott $A$-connection $\nabla$ on $B$, we also have a $B$-“connection" on $A$ :

$$
\Gamma(B) \otimes \Gamma(A) \rightarrow \Gamma(A):(u, a) \mapsto \Delta_{u} a=\operatorname{pr}_{A}[u, a]_{L} .
$$

Introduce the following maps:

$$
\begin{array}{rll}
\rho_{B}: & B \rightarrow T_{M}, \quad \rho_{B}=\left.\rho_{L}\right|_{B}, \\
{[-,-]_{B}:} & \Gamma(B) \otimes \Gamma(B) \rightarrow \Gamma(B), \quad\left[u_{1}, u_{2}\right]_{B}=\operatorname{pr}_{B}\left[u_{1}, u_{2}\right]_{L}, \\
\beta(-,-): & \Gamma(B) \otimes \Gamma(B) \rightarrow \Gamma(A), \quad & \beta\left(u_{1}, u_{2}\right)=\operatorname{pr}_{A}\left[u_{1}, u_{2}\right]_{L},
\end{array}
$$

$\forall u_{1}, u_{2} \in \Gamma(B)$. The Lie algebroid structure on $L$ can be described as follows:

$$
\left\{\begin{array}{l}
{\left[a_{1}, a_{2}\right]_{L}=\left[a_{1}, a_{2}\right]_{A} ;} \\
{\left[u_{1}, u_{2}\right]_{L}=\beta\left(u_{1}, u_{2}\right)+\left[u_{1}, u_{2}\right]_{B}} \\
{[a, u]_{L}=-\Delta_{u} a+\nabla_{a}^{\text {Bott }} u} \\
\rho_{L}(a+u)=\rho_{A}(a)+\rho_{B}(u),
\end{array}\right.
$$

$\forall a, a_{1}, a_{2} \in \Gamma(A), u, u_{1}, u_{2} \in \Gamma(B)$.

Let $\Omega_{A}^{p}\left(\Lambda^{q} B^{\vee}\right)=\Gamma\left(\Lambda^{p} A^{\vee} \otimes \Lambda^{q} B^{\vee}\right)$. Then

$$
C^{\infty}(\mathcal{L}[1])=\Gamma\left(\Lambda^{\bullet} L^{\vee}[-1]\right) \cong \oplus_{p \geq 0, q \geq 0} \Omega_{A}^{p}\left(\Lambda^{q} B^{\vee}\right)[-p-q]
$$

Since $\wedge^{\bullet} B$ is an $A$-module, we have the standard Chevalley-Eilenberg differential

$$
d_{A}^{\text {Bott }}: \Omega_{A}^{p}\left(\Lambda^{q} B^{\vee}\right) \rightarrow \Omega_{A}^{p+1}\left(\Lambda^{q} B^{\vee}\right) .
$$

In a similar fashion, we can define a map

$$
d_{B}^{\Delta}: \Omega_{A}^{p}\left(\Lambda^{q} B^{\vee}\right) \rightarrow \Omega_{A}^{p}\left(\Lambda^{q+1} B^{\vee}\right) .
$$

Note that, however, $d_{B}^{\Delta}$ may not square to zero. There is also a degree $(+1)$ derivation:

$$
d_{\beta}: \Omega_{A}^{p}\left(\Lambda^{q} B^{\vee}\right) \rightarrow \Omega_{A}^{p-1}\left(\Lambda^{q+2} B^{\vee}\right),
$$

generated by the following relations:

$$
\begin{gathered}
d_{\beta} f=0, \quad \forall f \in C^{\infty}(M) ; \\
\left\langle d_{\beta}(\omega), u_{1} \wedge u_{2}\right\rangle=-\left\langle\omega, \beta\left(u_{1}, u_{2}\right)\right\rangle, \quad \forall \omega \in \Gamma\left(A^{\vee}\right), u_{1}, u_{2} \in \Gamma(B) .
\end{gathered}
$$

The following lemma is immediate.

Lemma 4.4. Under the isomorphism (19), the homological vector field $Q$ on $\mathcal{L}[1]$ is given by

$$
d_{L}=d_{A}^{\text {Bott }}+d_{B}^{\Delta}+d_{\beta} .
$$

Consider the vector bundle of $\mathbb{Z}$-graded manifolds $\mathcal{L}=A[1] \oplus B \rightarrow A[1]$. Its space of sections $\Gamma(\mathcal{L})$ is isomorphic to $\Omega_{A}^{\bullet}(B)$. The space of vector fields on $A[1]$ is $\operatorname{Der}\left(\Omega_{A}^{\bullet}\right)$.

Lemma 4.5. The $L_{\infty}$ algebroid structure on $\mathcal{L} \rightarrow A[1]$, where $\mathcal{L}=A[1] \oplus B$, as in Proposition 4.1 is determined by the following relations:

(1) The 0th anchor $\rho_{0}=d_{A}: \Omega_{A}^{\bullet} \rightarrow \Omega_{A}^{\bullet+1}$ is the Chevalley-Eilenberg differential of the Lie algebroid A.

(2) The unary anchor $\rho_{1}: \mathcal{L} \rightarrow T_{A[1]}$ is determined by

$$
\rho_{1}(u)=\Delta_{u}, \quad \forall u \in \Gamma(B) .
$$

(3) The binary anchor $\rho_{2}: \wedge^{2} \mathcal{L} \rightarrow T_{A[1]}$ is determined by

$$
\rho_{2}\left(u_{1}, u_{2}\right)=i_{\beta\left(u_{1}, u_{2}\right)}, \quad \forall u_{1}, u_{2} \in \Gamma(B) .
$$


(4) The unary bracket $l_{1}$ coincides with the Chevalley-Eilenberg differential $d_{A}^{\text {Bott }}: \Omega_{A}^{\bullet}(B) \rightarrow \Omega_{A}^{\bullet+1}(B)$.

(5) The binary bracket $l_{2}: \wedge^{2} \Gamma(\mathcal{L}) \rightarrow \Gamma(\mathcal{L})$ is determined by

$$
l_{2}\left(u_{1}, u_{2}\right)=\left[u_{1}, u_{2}\right]_{B}, \quad \forall u_{1}, u_{2} \in \Gamma(B) .
$$

(6) The ternary bracket $l_{3}: \wedge^{3} \Gamma(\mathcal{L}) \rightarrow \Gamma(\mathcal{L})$ is determined by

$$
l_{3}\left(u_{1}, u_{2}, u_{3}\right)=0, \quad \forall u_{1}, u_{2}, u_{3} \in \Gamma(B) .
$$

(7) All higher brackets $l_{i}(i \geq 4)$, and anchors $\rho_{j}(j \geq 3)$ vanish.

Proof. We follow the construction as in the proof of Proposition A.1. The relevant Voronov data are as follows:

(1) The graded Lie algebra of first order differential operators on $\mathcal{L}[1]$ is

$$
\mathfrak{A}=\mathscr{D} \leq 1(\mathcal{L}[1]) \cong \operatorname{Der}\left(\Omega_{A}^{\bullet}\left(\Lambda^{\bullet} B^{\vee}\right)\right) \oplus \Omega_{A}^{\bullet}\left(\Lambda^{\bullet} B^{\vee}\right) .
$$

Here elements in $\Omega_{A}^{\bullet}\left(\Lambda^{\bullet} B^{\vee}\right)=C^{\infty}(\mathcal{L}[1])$ are considered as zeroth order differentials, i.e. the multiplication by functions.

(2) The abelian Lie subalgebra $\mathfrak{a}=\Gamma(\mathcal{L}[1]) \oplus C^{\infty}(A[1]) \cong \Omega_{A}^{\bullet}(B) \oplus \Omega_{A}^{\bullet}$.

The inclusion $\mathfrak{a} \hookrightarrow \mathfrak{A}$ is given as follows: $\forall a \in \Omega_{A}^{r}(B), \iota_{a}$ is the contraction operator

$$
\iota_{a}: \Omega_{A}^{p}\left(\Lambda^{q} B^{\vee}\right) \rightarrow \Omega_{A}^{p+r}\left(\Lambda^{q-1} B^{\vee}\right) .
$$

Here $\Omega_{A}^{\bullet}$ is considered as a subalgebra in $\Omega_{A}^{\bullet}\left(\Lambda^{\bullet} B^{\vee}\right)$ naturally.

(3) The projection map $P: \mathfrak{A} \rightarrow \mathfrak{a}$ is given by

$$
P(v+\mu)=P_{1}(v)+P_{2}(\mu), \quad \forall v \in \operatorname{Der}\left(\Omega_{A}^{\bullet}\left(\Lambda^{\bullet} B^{\vee}\right)\right), \mu \in \Omega_{A}^{\bullet}\left(\Lambda^{\bullet} B^{\vee}\right),
$$

where $P_{2}: \Omega_{A}^{\bullet}\left(\Lambda^{\bullet} B^{\vee}\right) \rightarrow \Omega_{A}^{\bullet}$ is the natural projection, and $P_{1}: \operatorname{Der}\left(\Omega_{A}^{\bullet}\left(\Lambda^{\bullet} B^{\vee}\right)\right) \rightarrow \Omega_{A}^{\bullet}(B)$ is defined as follows: $\forall v \in \operatorname{Der}\left(\Omega_{A}^{\bullet}\left(\Lambda^{\bullet} B^{\vee}\right)\right), P_{1}(v) \in \Omega_{A}^{\bullet}(B)$ is determined by the composition

$$
\Gamma\left(B^{\vee}\right) \stackrel{v}{\rightarrow} \Omega_{A}^{\bullet}\left(\Lambda^{\bullet} B^{\vee}\right) \stackrel{\mathrm{pr}}{\rightarrow} \Omega_{A}^{\bullet} .
$$

(4) The homological vector field $Q \in \mathfrak{A}$ on $\mathcal{L}[1]$ is $d_{L}=d_{A}^{\text {Bott }}+d_{B}^{\Delta}+d_{\beta}$ as in Equation (20).

We now apply Equations (38) and (37) to obtain structure maps of the $L_{\infty}$ algebroid structure on $\mathcal{L} \rightarrow A[1]$. For this purpose, observe that, in the decomposition $d_{L}=d_{A}^{\text {Bott }}+d_{B}^{\Delta}+d_{\beta}$, the operator $d_{A}^{\text {Bott }}$ is of weight $0, d_{B}^{\Delta}$ is of weight 1 , and $d_{\beta}$ is of weight 2 .

Applying Equation (37), the 0th anchor is given by

$$
\rho_{0}(\omega)=P_{2}\left[d_{L}, \omega\right]=\left[d_{A}^{\text {Bott }}, \omega\right]=d_{A}(\omega), \quad \forall \omega \in \Omega_{A}^{\bullet} .
$$

This proves (1). The unary anchor can be obtained by the same method:

$$
\begin{aligned}
\rho_{1}(u)(\omega) & =P_{2}\left[\left[d_{L}, \iota_{u}\right], \omega\right]=\left[\left[d_{B}^{\Delta}, \iota_{u}\right], \omega\right] \\
& =\left(d_{B}^{\Delta} \circ \iota_{u}+\iota_{u} \circ d_{B}^{\Delta}\right) \omega \\
& =\iota_{u} d_{B}^{\Delta} \omega=\Delta_{u} \omega, \quad \forall \omega \in \Omega_{A}^{\bullet} .
\end{aligned}
$$

This proves (2). One can prove (3) similarly.

Next we describe the multi-brackets. For the unary bracket, we apply Equation (38). For any $u \in \Gamma(B)$, note that $l_{1}(u) \in \Omega_{A}^{1}(B)$. For any $\eta \in \Omega_{A}^{0}\left(\Lambda^{1} B^{\vee}\right)=\Gamma\left(B^{\vee}\right)$, we have

$$
\begin{aligned}
\iota_{l_{1}(u)}(\eta) & =\left(P_{1}\left[d_{L}, \iota_{u}\right]\right)(\eta)=\left[d_{A}^{\text {Bott }}, \iota_{u}\right](\eta) \\
& =\left(d_{A}^{\text {Bott }} \circ \iota_{u}+\iota_{u} \circ d_{A}^{\text {Bott }}\right)(\eta) \\
& =\iota_{d_{A}^{\text {Bott }} u}(\eta) .
\end{aligned}
$$

This proves (4). 
For the binary bracket, let $u_{1}, u_{2} \in \Gamma(B), \eta \in \Gamma\left(B^{\vee}\right)$. Then,

$$
\begin{aligned}
\iota_{l_{2}\left(u_{1}, u_{2}\right)}(\eta) & =\left(P_{1}\left[\left[d_{L}, \iota_{u_{1}}\right], \iota_{u_{2}}\right]\right)(\eta)=\left[\left[d_{B}^{\Delta}, \iota_{u_{1}}\right], \iota_{u_{2}}\right](\eta) \\
& =\left(d_{B}^{\Delta} \circ \iota_{u_{1}} \circ \iota_{u_{2}}+\iota_{u_{1}} \circ d_{B}^{\Delta} \circ \iota_{u_{2}}-\iota_{u_{2}} \circ d_{B}^{\Delta} \circ \iota_{u_{1}}-\iota_{u_{2}} \circ \iota_{u_{1}} \circ d_{B}^{\Delta}\right)(\eta) \\
& =\iota_{u_{1}} d_{B}^{\Delta}\left\langle u_{2}, \eta\right\rangle-\iota_{u_{2}} d_{B}^{\Delta}\left\langle u_{1}, \eta\right\rangle-\iota_{u_{1}} \iota_{u_{2}} d_{B}^{\Delta}(\eta) \\
& =\left\langle\eta,\left[u_{1}, u_{2}\right]_{B}\right\rangle=\iota_{\left[u_{1}, u_{2}\right]_{B}}(\eta) .
\end{aligned}
$$

This proves (5). The rest of claims (6) and (7) can be proved in a similar fashion.

Proof of Proposition 4.3. According to Theorem 3.8, the $L_{\infty}$ algebroid $\mathcal{L} \rightarrow A[1]$ induces a degree $(+1)$ derived Poisson algebra structure on the space of sections $\Gamma(\hat{S}(\mathcal{L}[1])) \cong \operatorname{tot} \Omega_{A}^{\bullet}\left(\Lambda^{\bullet} B\right)$, whose multi-brackets are obtained from the structure maps on $\mathcal{L}$ by applying the Leibniz rule. The rest follows from a straightforward verification.

4.2. Second construction: Fedosov dg Lie algebroid arising from a Lie pair. Let us first recall Fedosov dg Lie algebroids as constructed in [2]. We will use the same settings as in Section 4.1: a Lie pair $(L, A)$ and $B=L / A$. Consider the graded vector bundle $\mathcal{M} \rightarrow L[1]$, where $\mathcal{M}=L[1] \oplus B$. It is clear that $C^{\infty}(\mathcal{M}) \cong \Gamma\left(\Lambda^{\bullet} L^{\vee} \otimes \hat{S} B^{\vee}\right)$.

Given a splitting of the short exact sequence (15), the following maps are established in $[2,48]$

- $\delta: \Gamma\left(\Lambda^{\bullet} L^{\vee} \otimes \hat{S} B^{\vee}\right) \rightarrow \Gamma\left(\Lambda^{\bullet+1} L^{\vee} \otimes \hat{S} B^{\vee}\right)$, a degree $(+1)$ derivation;

- $\sigma: \Gamma\left(\Lambda^{\bullet} L^{\vee} \otimes \hat{S} B^{\vee}\right) \rightarrow \Gamma\left(\Lambda^{\bullet} A^{\vee}\right)$, the projection;

- $\tau: \Gamma\left(\Lambda^{\bullet} A^{\vee}\right) \rightarrow \Gamma\left(\Lambda^{\bullet} L^{\vee} \otimes \hat{S} B^{\vee}\right)$, the inclusion;

- $h: \Gamma\left(\Lambda^{\bullet} L^{\vee} \otimes \hat{S} B^{\vee}\right) \rightarrow \Gamma\left(\Lambda^{\bullet-1} L^{\vee} \otimes \hat{S} B^{\vee}\right)$, the homotopy map;

- $\sigma_{\natural}=\sigma \otimes 1: \Gamma\left(\Lambda^{\bullet} L^{\vee} \otimes \hat{S} B^{\vee} \otimes \Lambda^{\bullet} B\right) \rightarrow \Gamma\left(\Lambda^{\bullet} A^{\vee} \otimes \Lambda^{\bullet} B\right)$, the projection;

- $\tau_{\natural}=\tau \otimes 1: \Gamma\left(\Lambda^{\bullet} A^{\vee} \otimes \Lambda^{\bullet} B\right) \rightarrow \Gamma\left(\Lambda^{\bullet} L^{\vee} \otimes \hat{S} B^{\vee} \otimes \Lambda^{\bullet} B\right)$, the inclusion;

- $h_{\natural}=h \otimes 1: \Gamma\left(\Lambda^{\bullet} L^{\vee} \otimes \hat{S} B^{\vee} \otimes \Lambda^{\bullet} B\right) \rightarrow \Gamma\left(\Lambda^{\bullet-1} L^{\vee} \otimes \hat{S} B^{\vee} \otimes \Lambda^{\bullet} B\right)$, the homotopy map.

We recall a result in [48]:

Proposition 4.6 (Theorem 2.5 in [48]). Let $(L, A)$ be a Lie pair. Given a splitting of the short exact sequence (15) and a torsion-free $L$-connection $\nabla$ on $B$ extending the Bott A-connection, there exists a unique 1-form valued in formal vertical vector fields of $B$ :

$$
X^{\nabla} \in \Gamma\left(L^{\vee} \otimes \hat{S}^{\geqslant 2} B^{\vee} \otimes B\right)
$$

satisfying $h_{\natural}\left(X^{\nabla}\right)=0$ and such that the derivation $Q: \Gamma\left(\Lambda^{\bullet} L^{\vee} \otimes \hat{S} B^{\vee}\right) \rightarrow \Gamma\left(\Lambda^{\bullet+1} L^{\vee} \otimes \hat{S} B^{\vee}\right)$ defined by

$$
Q=-\delta+d_{L}^{\nabla}+X^{\nabla}
$$

satisfies $Q^{2}=0$. Here

(1) $d_{L}^{\nabla}: \Gamma\left(\Lambda^{\bullet} L^{\vee} \otimes \hat{S} B^{\vee}\right) \rightarrow \Gamma\left(\Lambda^{\bullet+1} L^{\vee} \otimes \hat{S} B^{\vee}\right)$ is the covariant derivative associated with the $L$ connection $\nabla$ on $B$;

(2) $X^{\nabla}$ acts on the algebra $\Gamma\left(\Lambda^{\bullet} L^{\vee} \otimes \hat{S} B^{\vee}\right)$ as a derivation in a natural fashion.

As a consequence, $(\mathcal{M}=L[1] \oplus B, Q)$ is a dg manifold, called the Fedosov dg manifold [48]. Consider the surjective submersion $\mathcal{M} \rightarrow M$. Let $\mathcal{F} \rightarrow \mathcal{M}$ denote the pullback of the vector bundle $B \rightarrow M$ through $\mathcal{M} \rightarrow M$. It is a graded vector bundle whose total space $\mathcal{F}$ is the graded manifold with support $M$ associated with the graded vector bundle $L[1] \oplus B \oplus B \rightarrow M$. Its space of sections $\Gamma(\mathcal{F} \rightarrow \mathcal{M})$ is canonically identified with $C^{\infty}(\mathcal{M}) \otimes_{C^{\infty}(M)} \Gamma(B)=\Gamma\left(\Lambda^{\bullet} L^{\vee} \otimes \hat{S}\left(B^{\vee}\right) \otimes B\right)$. It is naturally a vector subbundle of $T_{\mathcal{M}} \rightarrow \mathcal{M}$; 
the inclusion $\Gamma(\mathcal{F} \rightarrow \mathcal{M}) \hookrightarrow \mathscr{X}(\mathcal{M})$ takes the section $\left(\lambda \otimes \chi^{J}\right) \otimes \partial_{k} \in C^{\infty}(\mathcal{M}) \otimes_{C^{\infty}(M)} \Gamma(B)$ of the vector bundle $\mathcal{F} \rightarrow \mathcal{M}$ to the derivation

$$
\mu \otimes \chi^{M} \mapsto \sum_{i} \lambda \wedge \mu \otimes M_{i} \chi^{J+M-e_{i}}
$$

of $C^{\infty}(\mathcal{M})$. Here $M=\left(\cdots M_{i} \cdots\right)$ denotes a multi-index, and $e_{i}$ denotes the multi-index all of whose components are 0 except for the $i$ th which is equal to 1 . It is simple to see that $\mathcal{F} \subset T_{\mathcal{M}}$ is a dg foliation of the dg manifold $(\mathcal{M}, Q)$, which is called the Fedosov dg Lie algebroid [2]. Hence $\left(\Gamma\left(\mathcal{M} ; \wedge^{\bullet} \mathcal{F}\right), \mathcal{L}_{Q}\right)$ is a $(+1)$-shifted derived Poisson algebra (or a differential Gerstenhaber algebra), where $\Gamma\left(\mathcal{M} ; \wedge^{\bullet} \mathcal{F}\right)=$ $\oplus_{k} \Gamma\left(\mathcal{M} ; \wedge^{k} \mathcal{F}\right)[k]$, and $\mathcal{L}_{Q}=[Q,-]$ denotes the Lie derivative. Since $\Gamma\left(\mathcal{M} ; \Lambda^{\bullet} \mathcal{F}\right) \cong \Gamma\left(\Lambda^{\bullet} L^{\vee} \otimes \hat{S} B^{\vee} \otimes\right.$ $\left.\Lambda^{\bullet} B\right)=\oplus_{k} \Gamma\left(\Lambda^{\bullet} L^{\vee} \otimes \hat{S} B^{\vee} \otimes \Lambda^{k} B\right)[k]$, it thus follows that

$$
\left(\Gamma\left(\Lambda^{\bullet} L^{\vee} \otimes \hat{S} B^{\vee} \otimes \Lambda^{\bullet} B\right), \mathcal{L}_{Q}\right)
$$

is a $(+1)$-shifted derived Poisson algebra (or a differential Gerstenhaber algebra).

We need another result:

Proposition 4.7 ([2]). Under the same hypothesis as in Proposition 4.6, there is an induced contraction datum

$$
\left.\left.\breve{h}_{\natural}{\operatorname{ltot} \Gamma\left(\Lambda^{\bullet}\right.}^{\vee} L^{\vee} \otimes \hat{S} B^{\vee} \otimes \Lambda^{\bullet} B\right), \mathcal{L}_{Q}\right) \underset{\breve{\tau}_{\natural}}{\stackrel{\sigma_{\natural}}{\rightleftarrows}}\left(\operatorname{tot} \Omega_{A}^{\bullet}\left(\Lambda^{\bullet} B\right), d_{A}^{\text {Bott }}\right) .
$$

The maps $\breve{h}_{\natural}$ and $\breve{\tau}_{\natural}$ are defined by (see [2]):

$$
\begin{aligned}
& \breve{h}_{\natural}=h_{\natural}+\sum_{i=1}^{\infty}\left(h_{\natural} \circ \mathcal{L}_{\varrho}\right)^{i} h_{\natural}, \\
& \breve{\tau}_{\natural}=\tau_{\natural}+\sum_{i=1}^{\infty}\left(h_{\natural} \circ \mathcal{L}_{\varrho}\right)^{i} \tau_{\natural} .
\end{aligned}
$$

Remark 4.8. We remark that the above is indeed a semifull algebra contraction, in the sense of Definition 2.11. In fact, as explained in detail in [2, loc. cit.], the contraction $\left(\sigma_{\natural}, \breve{\tau}_{\natural}, \breve{h}_{\natural}\right)$ is obtained by applying the perturbation Lemma 2.10 to a second contraction involving the maps $\left(\sigma_{\natural}, \tau_{\natural}, h_{\natural}\right)$. The fact that the latter contraction is semifull can be shown by a simple direct computation, using Remark 2.12 and the explicit definition of the maps $\sigma_{\natural}, \tau_{\natural}, h_{\natural}$ given in [2]. The fact that the contraction $\left(\sigma_{\natural}, \breve{\tau}_{\natural}, \breve{h}_{\natural}\right)$ is semifull follows immediately from Proposition 2.14.

The differential Gerstenhaber algebra $\left(\Gamma\left(\Lambda^{\bullet} L^{\vee} \otimes \hat{S} B^{\vee} \otimes \Lambda^{\bullet} B\right), \mathcal{L}_{Q}\right)$ is, by definition, a degree $(+1)$ derived Poisson algebra, whose unary bracket is $\mathcal{L}_{Q}$, binary bracket is the Schouten bracket, and all higher brackets vanishes. By homotopy transfer Theorem 2.15, there is a degree $(+1)$ derived Poisson algebra structure induced on tot $\Omega_{A}^{\bullet}\left(\Lambda^{\bullet} B\right)$, whose first bracket is $d_{A}^{\text {Bott }}$.

Below is our main result in this section.

Proposition 4.9. Let $(L, A)$ be a Lie pair. Choose a splitting of the exact sequence (15), and a torsion free $L$ connection $\nabla$ on $B$ that extends the A-module structure of $B$. Then the degree $(+1)$ derived Poisson algebra structure on $\Omega_{A}^{\bullet}\left(\Lambda^{\bullet} B\right)$ obtained by homotopy transfer from the one on $\Gamma\left(\Lambda^{\bullet} L^{\vee} \otimes \hat{S} B^{\vee} \otimes \Lambda^{\bullet} B\right)$, which is induced from the Fedosov dg Lie algebroid $\mathcal{F} \rightarrow \mathcal{M}$, coincides with the one as in Proposition 4.3.

Note that the construction of a Fedosov manifold $\mathcal{M}$ and its Fedosov dg Lie algebroid $\mathcal{F} \rightarrow \mathcal{M}$ depends on the choice of a torsion free $L$-connection $\nabla$ on $B$ extending the $A$-module structure of $B$. The above 
proposition indicates that, however, the degree $(+1)$ derived Poisson algebra structure on $\Omega_{A}^{\bullet}\left(\Lambda^{\bullet} B\right)$ obtained by homotopy transfer from $\left(\Gamma\left(\mathcal{M} ; \wedge^{\bullet} \mathcal{F}\right), \mathcal{L}_{Q}\right)$ is independent of the choice of $\nabla$.

The rest of this section is devoted to prove this proposition. We need to recall some techniques and facts that are already shown in [2]. Let us resume the settings and notions in Section 4.1. We chose a splitting of Sequence (15) so that one treats $L=A \oplus B$ directly.

Given a torsion free $L$-connection $\nabla$ on $B$ that extends the $A$-module structure of $B$, one can write

$$
\nabla_{a+b} b^{\prime}=\nabla_{a}^{A} b^{\prime}+\Delta_{b}^{B} b^{\prime}, \quad \forall a \in \Gamma(A), b, b^{\prime} \in \Gamma(B) .
$$

Here the $\Delta^{B}$ can be thought of as a $B$-“connection" on $B$. The condition that $\nabla$ being torsion free means

$$
\Delta_{b_{1}}^{B} b_{2}-\Delta_{b_{2}}^{B} b_{1}=\left[b_{1}, b_{2}\right]_{B}, \quad \forall b_{1}, b_{2} \in \Gamma(B) .
$$

Here $[-,-]_{B}$ is the $B$-"bracket" introduced in Equation (18).

In what follows, let us denote

$$
C^{(p, q, r)}=\Gamma\left(\Lambda^{p} A^{\vee} \otimes \Lambda^{q} B^{\vee} \otimes S^{r} B^{\vee}\right) .
$$

Recall $\mathcal{M}=L[1] \oplus B$. Hence

$$
C^{\infty}(\mathcal{M})=\Gamma\left(\Lambda^{\bullet} L^{\vee} \otimes \hat{S} B^{\vee}\right)=\Gamma\left(\Lambda^{\bullet} A^{\vee} \otimes \Lambda^{\bullet} B^{\vee} \otimes \hat{S} B^{\vee}\right)=\prod_{p, q, r \geq 0} C^{(p, q, r)}
$$

Recall Equation (20), where we split $d_{L}$ into three components. Abusing notations, let us again write

$$
d_{L}^{\nabla}=d_{A}^{\text {Bott }}+d_{B}^{\Delta}+d_{\beta}: \Gamma\left(\Lambda^{\bullet} L^{\vee} \otimes \hat{S} B^{\vee}\right) \rightarrow \Gamma\left(\Lambda^{\bullet+1} L^{\vee} \otimes \hat{S} B^{\vee}\right),
$$

where

$$
\begin{gathered}
d_{A}^{\text {Bott }}: C^{(p, q, r)} \rightarrow C^{(p+1, q, r)}, \\
d_{B}^{\Delta}: C^{(p, q, r)} \rightarrow C^{(p, q+1, r)}, \\
d_{\beta}: C^{(p, q, r)} \rightarrow C^{(p-1, q+2, r)} .
\end{gathered}
$$

All the following are due to the relevant definitions and facts in [2].

- The kernel of $\sigma_{\natural}$ is $\left(C^{(\bullet, \geq 1, \bullet)} \oplus C^{(\bullet \bullet \bullet, \geq 1)}\right) \otimes \Gamma\left(\Lambda^{\bullet} B\right)$.

- The map $h_{\natural}$ sends $C^{(p, q, r)} \otimes \Gamma\left(\Lambda^{s} B\right)$ to $C^{(p, q-1, r+1)} \otimes \Gamma\left(\Lambda^{s} B\right)$.

- The map

$$
\varrho=d_{L}^{\nabla}+X^{\nabla}
$$

is a degree $(+1)$ derivation of $C^{\infty}(\mathcal{M})$, and in fact a perturbation of the cochain complex $\left(C^{\infty}(\mathcal{M}),-\delta\right)$. Moreover, the map $\mathcal{L}_{\varrho}$ satisfies

$$
\mathcal{L}_{\varrho}\left(C^{(p, q, r)} \otimes \Gamma\left(\Lambda^{s} B\right)\right) \subset\left(C^{(p+1, q, \geq r)} \oplus C^{(p, q+1, \geq r)} \oplus C^{(p-1, q+2, \geq r)}\right) \otimes \Gamma\left(\Lambda^{s} B\right) .
$$

- The Schouten-Nijenhuis bracket in $\Gamma\left(\Lambda^{\bullet} L^{\vee} \otimes \hat{S} B^{\vee} \otimes \Lambda^{\bullet} B\right)=C^{(\bullet, \bullet, \bullet)} \otimes \Gamma\left(\Lambda^{\bullet} B\right)$ satisfies

$$
\left[C^{(p, q, r)} \otimes \Gamma\left(\Lambda^{s} B\right), C^{(a, b, c)} \otimes \Gamma\left(\Lambda^{d} B\right)\right] \subset C^{(p+a, q+b, r+c-1)} \otimes \Gamma\left(\Lambda^{s+d-1} B\right) .
$$

Using these facts, the following formulas can be straightforward verified.

Lemma 4.10. 1) For any $b \in \Gamma(B)$, one has

$$
\breve{\tau}_{\natural}(b)=b+\sum_{i=1}^{\infty}\left(h_{\natural} \circ \mathcal{L}_{\varrho}\right)^{i}(b) \equiv b+h_{\natural}\left(d_{B}^{\Delta} b\right) \quad \bmod C^{(0,0, \geq 2)} \otimes \Gamma(B) .
$$

Note that $h_{\natural}\left(d_{B}^{\Delta} b\right) \in C^{(0,0,1)} \otimes \Gamma(B)$. 
2) For any $\theta \in \Gamma\left(A^{\vee}\right)$, one has

$$
\breve{\tau}_{\mathfrak{\natural}}(\theta)=\theta+\sum_{i=1}^{\infty}(h \circ \varrho)^{i}(\theta) \equiv \theta+h\left(d_{B}^{\Delta} \theta\right)+h\left(d_{\beta} \theta\right) \quad \bmod \left(C^{(1,0, \geq 2)} \oplus C^{(0,1, \geq 2)}\right) .
$$

Note that $h\left(d_{B}^{\Delta} \theta\right) \in C^{(1,0,1)}$ and $h\left(d_{\beta} \theta\right) \in C^{(0,1,1)}$.

3) For any $\varpi \in C^{(p, q, r)} \otimes \Gamma\left(\Lambda^{s} B\right)$, one has

$$
\breve{h}_{\natural}(\varpi) \equiv h_{\natural}(\varpi) \quad \bmod \bigoplus_{i+j=p+q-1} C^{(i, j, \geq r+2)} \otimes \Gamma\left(\Lambda^{s} B\right) .
$$

Note that $h_{\natural}(\varpi) \in C^{(p, q-1, r+1)} \otimes \Gamma\left(\Lambda^{s} B\right)$.

An immediate consequence is the following fact.

Lemma 4.11. For any $\varpi_{1}, \varpi_{2} \in C^{(\bullet, \bullet, \bullet)} \otimes \Gamma\left(\Lambda^{s} B\right)$, one has

$$
\sigma_{\natural}\left[\breve{h}_{\natural}\left(\varpi_{1}\right), \breve{h}_{\natural}\left(\varpi_{2}\right)\right]=0 .
$$

The following identities are due to the definition of $h$ (see [2]). We omit the details of verification.

Lemma 4.12. For all $b, b_{1}, b_{2} \in \Gamma(B), \theta \in \Gamma\left(A^{\vee}\right)$, we have

$$
\begin{aligned}
\iota_{b_{1}} h_{\natural}\left(d_{B}^{\Delta} b_{2}\right) & =\Delta_{b_{1}}^{B} b_{2}, \\
\iota_{b} h\left(d_{B}^{\Delta} \theta\right) & =\Delta_{b} \theta, \\
\iota_{b_{1}} h \iota_{b_{2}} h\left(d_{\beta} \theta\right) & =\frac{1}{2}\left\langle\beta\left(b_{1}, b_{2}\right), \theta\right\rangle .
\end{aligned}
$$

Here $\Delta$ is the B- "connection" on A introduced in Equation (17).

We are now able to show the following

Lemma 4.13. For $b_{1}, b_{2} \in \Gamma(B), \theta_{1}, \theta_{2} \in \Gamma\left(A^{\vee}\right)$ and $f \in C^{\infty}(M)$, one has

$$
\left\{\begin{array}{l}
\sigma_{\natural}\left[\breve{\tau}_{\natural}\left(b_{1}\right), \breve{\tau}_{\natural}\left(b_{2}\right)\right]=\left[b_{1}, b_{2}\right]_{B}, \\
\sigma_{\natural}\left[\breve{\tau}_{\natural}\left(b_{1}\right), \breve{\tau}_{\natural}\left(\theta_{1}\right)\right]=\Delta_{b_{1}} \theta_{1}, \\
\sigma_{\natural}\left[\breve{\tau}_{\natural}\left(b_{1}\right), f\right]=\rho_{B}\left(b_{1}\right) f, \\
\sigma_{\natural}\left[\breve{\tau}_{\natural}\left(\theta_{1}\right), \breve{\tau}_{\natural}\left(\theta_{2}\right)\right]=0 .
\end{array}\right.
$$

Proof. By Equation (24), we have

$$
\begin{aligned}
{\left[\breve{\tau}_{\natural}\left(b_{1}\right), \breve{\tau}_{\natural}\left(b_{2}\right)\right] } & \equiv \iota_{b_{1}} h_{\natural}\left(d_{B}^{\Delta} b_{2}\right)-\iota_{b_{2}} h_{\natural}\left(d_{B}^{\Delta} b_{1}\right) \bmod C^{(0,0, \geq 1)} \otimes \Gamma(B) \\
& \equiv \Delta_{b_{1}}^{B} b_{2}-\Delta_{b_{2}}^{B} b_{1} \bmod C^{(0,0, \geq 1)} \otimes \Gamma(B) \\
& \equiv\left[b_{1}, b_{2}\right]_{B} \bmod C^{(0,0, \geq 1)} \otimes \Gamma(B) .
\end{aligned}
$$

The last step is due to $\nabla$ being torsion free (Equation (23)). Applying $\sigma_{\natural}$, the first identity in Equation (28) is immediate.

Similarly, by Equations (24) and (25),

$$
\begin{aligned}
{\left[\breve{\tau}_{\natural}(b), \breve{\tau}_{\natural}(\theta)\right] } & \equiv \iota_{b} h\left(d_{B}^{\Delta} \theta\right)+\iota_{b} h\left(d_{\beta} \theta\right) \quad \bmod \left(C^{(1,0, \geq 1)} \oplus C^{(0,1, \geq 1)}\right) \\
& \equiv \Delta_{b} \theta+\iota_{b} h\left(d_{\beta} \theta\right) \quad \bmod \left(C^{(1,0, \geq 1)} \oplus C^{(0,1, \geq 1)}\right) .
\end{aligned}
$$

Notice that $\iota_{b} h\left(d_{\beta} \theta\right) \in C^{(0,1,0)}$. Then applying $\sigma_{\natural}$, the second identity in Equation (28) is immediate. The third identity easily follows from Equation (24):

$$
\left[\breve{\tau}_{\mathfrak{\natural}}\left(b_{1}\right), f\right] \equiv \rho_{B}\left(b_{1}\right) f \quad \bmod C^{(0,0, \geq 1)} .
$$

The last identity is obvious. 
Lemma 4.14. For $b, b_{1}, b_{2}, b_{3} \in \Gamma(B)$ and $\theta, \theta_{1}, \theta_{2}, \theta_{3} \in \Gamma\left(A^{\vee}\right)$, one has

$$
\left\{\begin{array}{l}
\sigma_{\natural}\left[\breve{\tau}_{\natural}\left(b_{1}\right), \breve{h}_{\natural}\left[\breve{\tau}_{\natural}\left(b_{2}\right), \breve{\tau}_{\natural}(\theta)\right]\right]=\frac{1}{2}\left\langle\beta\left(b_{1}, b_{2}\right), \theta\right\rangle, \\
\sigma_{\natural}\left[\breve{\tau}_{\natural}(\theta), \breve{h}_{\natural}\left[\breve{\tau}_{\natural}\left(b_{1}\right), \breve{\tau}_{\natural}\left(b_{2}\right)\right]\right]=0, \\
\sigma_{\natural}\left[\breve{\tau}_{\natural}\left(b_{1}\right), \breve{h}_{\natural}\left[\breve{\tau}_{\natural}\left(b_{2}\right), \breve{\tau}_{\natural}\left(b_{3}\right)\right]\right]=0, \\
\sigma_{\natural}\left[\breve{\tau}_{\natural}(b), \breve{h}_{\natural}\left[\breve{\tau}_{\natural}\left(\theta_{1}\right), \breve{\tau}_{\natural}\left(\theta_{2}\right)\right]\right]=0, \\
\sigma_{\natural}\left[\breve{\tau}_{\natural}\left(\theta_{1}\right), \breve{h}_{\natural}\left[\breve{\tau}_{\natural}\left(\theta_{2}\right), \breve{\tau}_{\natural}(b)\right]\right]=0, \\
\sigma_{\natural}\left[\breve{\tau}_{\natural}\left(\theta_{1}\right), \breve{h}_{\natural}\left[\breve{\tau}_{\natural}\left(\theta_{2}\right), \breve{\tau}_{\natural}\left(\theta_{3}\right)\right]\right]=0 .
\end{array}\right.
$$

Proof. We show the first identity in (31). By Equations (30) and (26), we have

$$
\breve{h}_{\natural}\left[\breve{\tau}_{\natural}\left(b_{2}\right), \breve{\tau}_{\natural}(\theta)\right] \equiv h \iota_{b_{2}} h\left(d_{\beta} \theta\right) \quad \bmod C^{(0,0, \geq 2)},
$$

where $h \iota_{b_{2}} h\left(d_{\beta} \theta\right) \in C^{(0,0,1)}$.

Therefore, using Equation (24), we get

$$
\begin{aligned}
{\left[\breve{\tau}_{\natural}\left(b_{1}\right), \breve{h}_{\natural}\left[\breve{\tau}_{\natural}\left(b_{2}\right), \breve{\tau}_{\natural}(\theta)\right]\right] } & \equiv \iota_{b_{1}} h \iota_{b_{2}} h\left(d_{\beta} \theta\right) \bmod C^{(0,0, \geq 1)} \\
& \equiv \frac{1}{2}\left\langle\beta\left(b_{1}, b_{2}\right), \theta\right\rangle \bmod C^{(0,0, \geq 1)} .
\end{aligned}
$$

Applying $\sigma_{\natural}$, one gets the first identity. The remaining identities can be worked out similarly.

The following lemma is proved along the same lines.

Lemma 4.15. The following equation holds for any $x, y, z \in \Gamma(B)$ or $\Gamma\left(A^{\vee}\right)$ :

$$
\breve{h}_{\natural}\left[\breve{\tau}_{\natural}(x), \breve{h}_{\natural}\left[\breve{\tau}_{\natural}(y), \breve{\tau}_{\natural}(z)\right]\right]=0 .
$$

With these preparatory work, we finally give the proof of our main result — Proposition 4.9.

Proof of Proposition 4.9. Via the contraction data $\left(\breve{h}_{\natural}, \sigma_{\natural}, \breve{\tau}_{\natural}\right)$ in Equation (22), one constructs the $L_{\infty}$ brackets on tot $\Omega_{A}^{\bullet}\left(\Lambda^{\bullet} B\right)$ (Theorem 2.15). The first one is already shown to be $d_{A}^{\text {Bott }}$.

According to the proof of Theorem 2.15, the binary bracket reads

$$
l_{2}(X, Y)=\sigma_{\natural}\left[\breve{\tau}_{\natural}(X), \breve{\tau}_{\natural}(Y)\right], \quad \forall X, Y \in \operatorname{tot} \Omega_{A}^{\bullet}\left(\Lambda^{\bullet} B\right) .
$$

Compare the identities in Equation (28) with those in (2) of Proposition 4.3, we see the generating relations of the binary bracket are exact the same.

The ternary bracket reads

$$
l_{3}(X, Y, Z)=\sigma_{\natural}\left[\breve{\tau}_{\natural}(X), \breve{h}_{\natural}\left[\breve{\tau}_{\natural}(Y), \breve{\tau}_{\natural}(Z)\right]\right]+c . p .
$$

Let us examine the ternary bracket on generating elements. For $b_{1}, b_{2} \in \Gamma(B)$ and $\theta \in \Gamma\left(A^{\vee}\right)$, we have

$$
\begin{aligned}
& l_{3}\left(b_{1}, b_{2}, \theta\right) \\
= & \sigma_{\natural}\left(\left[\breve{\tau}_{\natural}\left(b_{1}\right), \breve{h}_{\natural}\left[\breve{\tau}_{\natural}\left(b_{2}\right), \breve{\tau}_{\natural}(\theta)\right]-\left[\breve{\tau}_{\natural}\left(b_{2}\right), \breve{h}_{\natural}\left[\breve{\tau}_{\natural}\left(b_{1}\right), \breve{\tau}_{\natural}(\theta)\right]+\left[\breve{\tau}_{\natural}(\theta), \breve{h}_{\natural}\left[\breve{\tau}_{\natural}\left(b_{1}\right), \breve{\tau}_{\natural}\left(b_{2}\right)\right]\right]\right)\right.\right. \\
= & \left.\frac{1}{2}\left\langle\beta\left(b_{1}, b_{2}\right), \theta\right\rangle-\frac{1}{2}\left\langle\beta\left(b_{2}, b_{1}\right), \theta\right\rangle \text { (by Equation }(31)\right) \\
= & \left\langle\beta\left(b_{1}, b_{2}\right), \theta\right\rangle .
\end{aligned}
$$

For the same reasons, one sees that $l_{3}$ vanishes if restricted to $\Gamma(B) \times \Gamma(B) \times \Gamma(B), \Gamma(B) \times \Gamma\left(A^{\vee}\right) \times \Gamma\left(A^{\vee}\right)$ and $\Gamma\left(A^{\vee}\right) \times \Gamma\left(A^{\vee}\right) \times \Gamma\left(A^{\vee}\right)$. So, the generating relations of the ternary bracket are exact the same as those in (3) of Proposition 4.3. 
The 4th-bracket reads

$$
\begin{aligned}
& l_{4}(X, Y, Z, W) \\
&=\lambda \sigma_{\natural}\left[\breve{h}_{\natural}\left[\breve{\tau}_{\natural}(X), \breve{\tau}_{\natural}(Y)\right], \breve{h}_{\natural}\left[\breve{\tau}_{\natural}(Z), \breve{\tau}_{\natural}(W)\right]\right]+c . p . \\
& \\
&+\mu \sigma_{\natural}\left[\breve{\tau}_{\natural}(X), \breve{h}_{\natural}\left[\breve{\tau}_{\natural}(Y), \breve{h}_{\natural}\left[\breve{\tau}_{\natural}(Z), \breve{\tau}_{\natural}(W)\right]\right]\right]+c . p .
\end{aligned}
$$

where $\lambda, \mu$ are two constants. By Equations (27) and (32), we see that $l_{4}$ vanishes if restricted to generating elements in $\Gamma(B)$ or $\Gamma\left(A^{\vee}\right)$. Hence $l_{4}$ is trivial. It can be similarly verified that all higher brackets $l_{j}(j \geq 5)$ are trivial.

This completes the proof.

4.3. Third construction: Dirac deformation and proof of main theorems. Deformation of Dirac structures has been studied at least 15 years ago by Severa [50] and Roytenberg [44]. It is well known that the deformation is controlled by an $L_{\infty}$ algebra, which is canonical up to $L_{\infty}$ isomorphisms. In fact, it is a degree $(-1)$ derived Poisson algebra. Let us recall the construction below.

Let $E$ be a Courant algebroid of signature $(n, n)$ over a smooth manifold $M$, and $D \subset E$ a Dirac structure. Choose a transversal almost Dirac (i.e. maximal isotropic) subbundle $C \subset E$ such that $E \cong D \oplus C$. Identify $C$ with $D^{\vee}$. Then we have $E \cong D \oplus D^{\vee}$.

The Courant bracket $[-,-]_{E}$ on $\Gamma(E)$ and the anchor map $\rho_{E}: E \rightarrow T_{M}$ induce, by restrictions, a skewsymmetric bracket $[-,-]_{D^{\vee}}$ on $\Gamma\left(D^{\vee}\right)$ and an anchor map $\rho_{\vee}: D^{\vee} \rightarrow T_{M}$,

$$
\begin{aligned}
& {[\xi, \eta]_{D^{\vee}}=\operatorname{pr}_{D^{\vee}}[\xi, \eta]_{E}, \quad \forall \xi, \eta \in \Gamma\left(D^{\vee}\right),} \\
& \rho_{\vee}=\left.\rho_{E}\right|_{D^{\vee}} .
\end{aligned}
$$

Let $\phi \in \Gamma\left(\Lambda^{3} D\right)$ be the section defined by

$$
\phi(\xi, \eta, \zeta)=2\left\langle[\xi, \eta]_{E}, \zeta\right\rangle_{E}=2\left\langle\operatorname{pr}_{D}[\xi, \eta]_{E}, \zeta\right\rangle_{E}, \quad \forall \xi, \eta, \zeta \in \Gamma\left(D^{\vee}\right)
$$

Here $\langle-,-\rangle_{E}$ is the symmetric metric on $E$. It can be easily verified that $\phi$ is indeed skew-symmetric.

Unless $C \cong D^{\vee}$ is again a Dirac structure, in general $\phi$ is non-zero and $\left(D^{\vee},[-,-]_{D^{\vee}}, \rho \vee\right)$ is not a Lie algebroid. Instead, $\left(D, D^{\vee}\right)$ forms a quasi-Lie bialgebroid [44].

Consider the graded algebra $\Gamma\left(\Lambda^{\bullet} D^{\vee}\right)=\oplus_{k=0} \Gamma\left(\wedge^{k} D^{\vee}\right)[-k]$, whose degree $n$-part is $\Gamma\left(\Lambda^{n} D^{\vee}\right)$, for $n \geq 0$. Let

$$
\lambda_{1}=d_{D}: \Gamma\left(\Lambda^{i} D^{\vee}\right) \rightarrow \Gamma\left(\Lambda^{i+1} D^{\vee}\right)
$$

be the Chevalley-Eilenberg differential of the Lie algebroid $D$.

Define a binary bracket

$$
\lambda_{2}: \Gamma\left(\Lambda^{i} D^{\vee}\right) \otimes \Gamma\left(\Lambda^{l} D^{\vee}\right) \rightarrow \Gamma\left(\Lambda^{i+l-1} D^{\vee}\right)
$$

by extending, using Leibniz rule (see Equation (1), for $n=2, k=-1$ ), the relation

$$
\lambda_{2}(\xi, \eta)=[\xi, \eta]_{D^{\vee}}, \quad \lambda_{2}(\xi, f)=\rho_{\vee}(\xi)(f), \quad \forall \xi, \eta \in \Gamma\left(D^{\vee}\right), f \in C^{\infty}(M) .
$$

Similarly, let

$$
\lambda_{3}: \Gamma\left(\Lambda^{i} D^{\vee}\right) \otimes \Gamma\left(\Lambda^{l} D^{\vee}\right) \otimes \Gamma\left(\Lambda^{r} D^{\vee}\right) \rightarrow \Gamma\left(\Lambda^{i+l+r-3} D^{\vee}\right)
$$

be the ternary bracket extending $\phi$, by Leibniz rule, in each argument ( $\lambda_{3}$ vanishes if one of the argument is a function on $M$ ).

The following result is due to Severa [50] and Roytenberg [44]. Relevant results appeared in [23] and more recently in [15].

Proposition 4.16. Let $E$ be a Courant algebroid of signature $(n, n)$ over a smooth manifold $M$, and $D \subset E$ a Dirac structure. Choose a transversal almost Dirac structure $C$. Then 
- $\Gamma\left(\Lambda^{\bullet} D^{\vee}\right)$, together with $\lambda_{1}, \lambda_{2}, \lambda_{3}$ defined above and $\lambda_{l}=0, l>3$, and the wedge product, is a degree $(-1)$ derived Poisson algebra.

- The underlying $L_{\infty}$ algebra structure on $\Gamma\left(\Lambda^{\bullet} D^{\vee}\right)[1]$ controls deformations of the Dirac structures $D \subset E$ in the following sense: the graph $\left\{X+\omega^{b}(X) \mid X \in D\right\} \subset E$ of an element $\omega \in \Gamma\left(\Lambda^{2} D^{\vee}\right)[1]$ is a Dirac structure if and only if $\omega$ satisfies the Maurer-Cartan equation:

$$
\lambda_{1}(\omega)+\frac{1}{2} \lambda_{2}(\omega, \omega)+\frac{1}{6} \lambda_{3}(\omega, \omega, \omega)=0
$$

In fact, such a degree $(-1)$ derived Poisson algebra structure on $\Gamma\left(\Lambda^{\bullet} D^{\vee}\right)$ is canonical, up to isomorphisms. Note that there is a one-one correspondence between almost Dirac structures transversal to $D$ and elements in $\Gamma\left(\Lambda^{2} D\right)$. Their relation is established as follows:

$$
\pi \in \Gamma\left(\Lambda^{2} D\right) \quad \leftrightarrow \quad C_{\pi}=\left\{\pi^{\sharp}(\xi)+\xi \mid \xi \in C \cong D^{\vee}\right\} .
$$

Proposition 4.17 ([49]). Under the same hypothesis as in Proposition 4.16, assume that $C_{\pi}$ is another almost Dirac structure transversal to $D$, which corresponds to an element $\pi \in \Gamma\left(\Lambda^{2} D\right)$. Then the $(-1)$ derived Poisson algebra structures on $\Gamma\left(\Lambda^{\bullet} D^{\vee}\right)$ induced from $C$ and $C_{\pi}$ are isomorphic. The isomorphism is given by $\exp \delta_{\pi}$, where $\delta_{\pi}$ is a coderivation on $\bar{S}\left(\Gamma\left(\Lambda^{\bullet} D^{\vee}\right)[2]\right.$ generated by

$$
\begin{aligned}
& S^{2}\left(\Gamma\left(\Lambda^{\bullet} D^{\vee}\right)\right) \rightarrow \Gamma\left(\Lambda^{\bullet} D^{\vee}\right) \\
& \xi \otimes \eta \mapsto \iota_{\pi}(\xi) \wedge \eta+\xi \wedge \iota_{\pi}(\eta)-\iota_{\pi}(\xi \wedge \eta) .
\end{aligned}
$$

Note that the bilinear map in Equation (33) is a biderivation of the graded commutative algebra $\Gamma\left(\Lambda^{\bullet} D^{\vee}\right)$ with respect to the wedge product. Therefore $\exp \delta_{\pi}$ is indeed compatible with respect to the associative algebra structure, according to Proposition 2.8.

Now consider a Lie pair $(L, A)$. Let $E=L \oplus L^{\vee}$ be the standard Courant algebroid [32], where the Courant bracket and the anchor map are defined, respectively, by

$$
\begin{aligned}
{[X+\alpha, Y+\beta]_{E} } & =[X, Y]_{L}+\left(L_{X} \beta-\iota_{Y} d_{L} \alpha\right), \\
\rho_{E}(X+\alpha) & =\rho_{L}(X),
\end{aligned}
$$

$\forall X+\alpha, Y+\beta \in \Gamma\left(L \oplus L^{\vee}\right)$. The symmetric pairing is:

$$
\langle X+\alpha, Y+\beta\rangle_{E}=\frac{1}{2}(\langle X, \beta\rangle+\langle Y, \alpha\rangle) \text {. }
$$

It is a standard result that $D=A \oplus A^{\perp}$ is a Dirac structure of $E$ [32]. Choose a splitting of the exact sequence (15) so that $L \cong A \oplus B$. Then $A^{\perp} \cong B^{\vee}$ and $B \oplus A^{\vee}$ is a transversal almost Dirac structure of $D$ in $E$. That is

$$
E \cong\left(A \oplus B^{\vee}\right) \oplus\left(B \oplus A^{\vee}\right) \cong D \oplus D^{\vee}
$$

with $D \cong A \oplus B^{\vee}$ and $D^{\vee} \cong B \oplus A^{\vee}$. Thus $\left(D, D^{\vee}\right)$ is a quasi-Lie bialgebroid, and the induced structure maps on $D^{\vee}$ are, respectively, given by the following relations:

1) The bracket $[-,-]_{D} \vee$ reads

$$
\begin{aligned}
{[u+\theta, v+\omega]_{D^{\vee}} } & =\operatorname{pr}_{D^{\vee}}\left([u, v]_{L}+\left(L_{u} \omega-\iota_{v} d_{L} \theta\right)\right) \\
& =\operatorname{pr}_{B}[u, v]_{L}+\operatorname{pr}_{A^{\vee}}\left(L_{u} \omega-L_{v} \theta\right),
\end{aligned}
$$

where $u+\theta, v+\omega \in \Gamma\left(D^{\vee}\right)=\Gamma\left(B \oplus A^{\vee}\right)$.

2) The anchor $\rho_{\vee}$ is simply

$$
\rho_{\vee}(u+\theta)=\rho_{L}(u) .
$$


3) The 3 -form $\phi$ on $D^{\vee}$ is:

$$
\begin{aligned}
\phi\left(u_{1}+\theta_{1}, u_{2}+\theta_{2}, u_{3}+\theta_{3}\right) & =2\left\langle\operatorname{pr}_{D}\left[u_{1}+\theta_{1}, u_{2}+\theta_{2}\right]_{E}, u_{3}+\theta_{3}\right\rangle_{E} \\
& =2\left\langle\operatorname{pr}_{A}\left[u_{1}, u_{2}\right]_{L}+\operatorname{pr}_{B^{\vee}}\left(L_{u_{1}} \theta_{2}-L_{u_{2}} \theta_{1}\right), u_{3}+\theta_{3}\right\rangle_{E} \\
& =\left\langle\operatorname{pr}_{A}\left[u_{1}, u_{2}\right]_{L}, \theta_{3}\right\rangle+\left\langle L_{u_{1}} \theta_{2}, u_{3}\right\rangle-\left\langle L_{u_{2}} \theta_{1}, u_{3}\right\rangle \\
& =\left\langle\operatorname{pr}_{A}\left[u_{1}, u_{2}\right]_{L}, \theta_{3}\right\rangle+\left\langle\operatorname{pr}_{A}\left[u_{3}, u_{1}\right]_{L}, \theta_{2}\right\rangle+\left\langle\operatorname{pr}_{A}\left[u_{2}, u_{3}\right]_{L}, \theta_{1}\right\rangle, \\
\forall u_{i} \in B, \theta_{i} \in A^{\vee}, i=1,2,3 . &
\end{aligned}
$$

The above generating relations determine a degree $(-1)$ derived Poisson algebra structure on

$$
\Gamma\left(\Lambda^{\bullet} D^{\vee}\right) \cong \Gamma\left(\Lambda^{\bullet}\left(B \oplus A^{\vee}\right)\right) \cong \oplus_{k=0, l=0} \Omega_{A}^{k}\left(\Lambda^{l} B\right)[-k-l] .
$$

In order to be consistent with the degree convention in Section 4.1 and Section 4.2, we now redesignate the degrees so that the subspace $\Omega_{A}^{k}\left(\Lambda^{l} B\right)$ is of degree $(k-l)$. In this way, we obtain the same $(+1)$ derived Poisson algebra on tot $\Omega_{A}^{\bullet}\left(\Lambda^{\bullet} B\right) \Omega_{A}^{k}\left(\Lambda^{l} B\right)[-k+l]$ as in Proposition 4.3, since they have exactly the same generating relations.

In summary, we have proved the following

Proposition 4.18. Let $(L, A)$ be a Lie pair, and $E=L \oplus L^{\vee}$ the standard Courant algebroid associated to the Lie algebroid $L$ [32]. Consider the Dirac structure $D=A \oplus A^{\perp} \cong A \oplus B^{\vee}$. Then

(1) A splitting of the exact sequence (15) determines an almost Dirac structure $B \oplus A^{\vee}$ transversal to $D$.

(2) The degree $(+1)$ derived Poisson algebra tot $\Omega_{A}^{\bullet}\left(\Lambda^{\bullet} B\right)$ corresponding to the transversal almost Dirac structure $B \oplus A^{\vee}$ as in Proposition 4.16 coincides with the one as in Proposition 4.3.

Proof of Theorem 1.1 and Theorem 1.2. According to Proposition 4.17 and Proposition 4.18, the degree $(+1)$ derived Poisson algebra tot $\Omega_{A}^{\bullet}\left(\Lambda^{\bullet} B\right)$ induced from Proposition 4.16, the one as in Proposition 4.3, and the one as in Proposition 4.9 all coincide, and is canonical up to derived Poisson algebra isomorphisms with the linear map being the identity. As a consequence, the induced degree $(+1)$ Poisson algebra, or a Gerstenhaber algebra, on the level of cohomology $\mathbb{H}\left(\Omega_{A}^{\bullet}\left(\Lambda^{\bullet} B\right), d_{A}^{\text {Bott }}\right)$, is indeed canonical.

\subsection{Examples.}

4.4.1. Matched pairs of Lie algebroids. Let $L$ be a Lie algebroid, $A$ and $B$ two Lie subalgebroids of $L$ such that $L \cong A \oplus B$ as vector bundles. Then $L / A \cong B$ is naturally an $A$-module, while $L / B \cong A$ is naturally a $B$-module. Then $(A, B)$ is said to form a matched pair. Alternatively, one can define a matched pair as follows.

Definition 4.19 ([33, 35, 37]). Lie algebroids $A$ and $B$ over the same base manifold $M$ are said to form a matched pair if there exists an action $\nabla$ of $A$ on $B$ and an action $\Delta$ of $B$ on $A$, such that the identities

$$
\begin{gathered}
{\left[\rho_{A}(X), \rho_{B}(Y)\right]=-\rho_{A}\left(\Delta_{Y} X\right)+\rho_{B}\left(\nabla_{X} Y\right),} \\
\nabla_{X}\left[Y_{1}, Y_{2}\right]=\left[\nabla_{X} Y_{1}, Y_{2}\right]+\left[Y_{1}, \nabla_{X} Y_{2}\right]+\nabla_{\Delta_{Y_{2}} X} Y_{1}-\nabla_{\Delta_{Y_{1}} X} Y_{2}, \\
\Delta_{Y}\left[X_{1}, X_{2}\right]=\left[\Delta_{Y} X_{1}, X_{2}\right]+\left[X_{1}, \Delta_{Y} X_{2}\right]+\Delta_{\nabla_{X_{2}} Y} X_{1}-\Delta_{\nabla_{X_{1}} Y} X_{2}
\end{gathered}
$$

hold for all $X_{1}, X_{2}, X \in \Gamma(A)$ and $Y_{1}, Y_{2}, Y \in \Gamma(B)$. Here $\rho_{A}$ and $\rho_{B}$ denote the anchor maps of $A$ and $B$ respectively.

Given a matched pair $(A, B)$ of Lie algebroids, there is a Lie algebroid structure on the direct sum vector bundle $L=A \oplus B$, with anchor

$$
X \oplus Y \mapsto \rho_{A}(X)+\rho_{B}(Y)
$$


and the Lie bracket

$$
\left[X_{1} \oplus Y_{1}, X_{2} \oplus Y_{2}\right]=\left(\left[X_{1}, X_{2}\right]+\Delta_{Y_{1}} X_{2}-\Delta_{Y_{2}} X_{1}\right) \oplus\left(\left[Y_{1}, Y_{2}\right]+\nabla_{X_{1}} Y_{2}-\nabla_{X_{2}} Y_{1}\right) .
$$

Clearly, the pair $(L, A)$ is a Lie pair, and the Bott $A$-connection on $L / A \cong B$ coincides with $\nabla$. Applying Proposition 4.3, we see that the ternary bracket vanishes, and tot $\Omega_{A}^{\bullet}\left(\Lambda^{\bullet} B\right)$ is in fact a dgla.

Theorem 4.20. For any given matched pair $(A, B)$ of Lie algebroids, tot $\Omega_{A}^{\bullet}\left(\Lambda^{\bullet} B\right)$ admits a canonical differential Gerstenhaber algebra structure ${ }^{2}$, where the multiplication is the wedge product, and the differential is the Chevalley-Eilenberg differential

$$
d_{A}^{\nabla}: \Omega_{A}^{\bullet}\left(\Lambda^{\bullet} B\right) \rightarrow \Omega_{A}^{\bullet+1}\left(\Lambda^{\bullet} B\right) .
$$

Here the A-module structure on $\Lambda^{\bullet} B$ is the natural extension of the A-action on $B$.

Theorem 4.21. For any given matched pair $(A, B)$ of Lie algebroids, the Chevalley-Eilenberg hypercohomology $\mathbb{H}\left(\Omega_{A}^{\bullet}\left(\Lambda^{\bullet} B\right), d_{A}^{\nabla}\right)$ admits a canonical Gerstenhaber algebra structure.

As an application, consider a complex manifold $X$. Set $A=T_{X}^{0,1}$ and $B=T_{X}^{1,0}$. Then $(A, B)$ is a matched pair of Lie algebroids over $\mathbb{C}$, and its direct sum $A \bowtie B$ is isomorphic, as a Lie algebroid, to $T_{X} \otimes \mathbb{C}$. It is simple to see that tot $\Omega_{A}^{\bullet}\left(\Lambda^{\bullet} B\right)=\operatorname{tot} \Omega_{X}^{0, \bullet}\left(T_{X}^{\bullet, 0}\right)$, the differential $d_{A}^{\nabla}$ is the standard $\bar{\partial}$-operator, and the Lie bracket $\lceil-,-\rceil$ is the Schouten bracket. Therefore, $\operatorname{tot}\left(\Omega_{X}^{0, \bullet}\left(T_{X}^{\bullet, 0}\right), \wedge,\lceil-,-\rceil, \bar{\partial}\right)$ is a differential Gerstenhaber algebra. The corresponding hypercohomology is isomorphic to the sheaf cohomology $\mathbb{H}\left(X, \Lambda^{\bullet} \Theta\right)$ of holomorphic polyvector fields. The Gerstenhaber algebra structure in Theorem 4.21 becomes the standard Gerstenhaber algebra structure on $\mathbb{H}\left(X, \Lambda^{\bullet} \Theta\right)$.

Another example of matched pairs arises from $\mathfrak{g}$-manifolds. Let $\mathfrak{g}$ be a Lie algebra, $M$ a $\mathfrak{g}$-manifold with infinitesimal action $\phi: \mathfrak{g} \rightarrow \mathscr{X}(M)$. Let $A=M \rtimes \mathfrak{g}$ be the associated action Lie algebroid and $B=T_{M}$. Then $A$ and $B$ form a Lie algebroid matched pair, with mutual actions

$$
\nabla_{a} Y=[\phi(a), Y], \quad \Delta_{Y} a=0,
$$

where $a \in \mathfrak{g}$ is considered as a constant section in $A$, and $Y \in \mathscr{X}(M)$. It is clear that tot $\Omega_{A}^{\bullet}\left(\Lambda^{\bullet} B\right) \cong$ tot $\Lambda^{\bullet} \mathfrak{g}^{\vee} \otimes \mathscr{X}_{\text {poly }}^{\bullet}(M)$, and $d_{A}^{\nabla}$ is the standard Chevalley-Eilenberg differential, and the Lie bracket $\lceil-,-\rceil$ is the extension of the Schouten bracket. Hence tot $\Lambda^{\bullet} \mathfrak{g}^{\vee} \otimes \mathscr{X}_{\text {poly }}^{\bullet}(M)$ is a differential Gerstenhaber algebra and its hypercohomology $\mathbb{H}_{\mathrm{CE}}\left(\mathfrak{g}, \mathscr{X}_{\text {poly }}^{\bullet}(M)\right)$ is a Gerstenhaber algebra. See [31, Lemma 3.1].

4.4.2. Semisimple Lie algebras. Let $\mathfrak{g}$ be a complex semisimple Lie algebra, and $\mathfrak{h}$ a Cartan subalgebra in $\mathfrak{g}$. Let $\mathfrak{g}=\mathfrak{h} \oplus \bigoplus_{\alpha \in \Delta} \mathfrak{g}_{\alpha}$ be its root decomposition, where $\Delta \subset \mathfrak{h}^{\vee}$ is the root system of $\mathfrak{g}$. It is standard that there exist $h_{\alpha} \in \mathfrak{h}$ and $x_{\alpha} \in \mathfrak{g}_{\alpha}$, such that

$$
\left[h_{\alpha}, x_{\alpha}\right]=2 x_{\alpha}, \quad\left[h_{\alpha}, x_{-\alpha}\right]=-2 x_{-\alpha}, \quad\left[x_{\alpha}, x_{-\alpha}\right]=h_{\alpha},
$$

for all $\alpha \in \Delta^{+}$.

Consider the Lie pair $(\mathfrak{g}, \mathfrak{h})$. Applying Theorems 1.1 and 1.2, we have the following

Theorem 4.22. Let $\mathfrak{g}$ be a complex semisimple Lie algebra with the root decomposition $\mathfrak{g}=\mathfrak{h} \oplus \bigoplus_{\alpha \in \Delta} \mathfrak{g}_{\alpha}$. Then tot $\Lambda^{\bullet} \mathfrak{h}^{\vee} \otimes \Lambda^{\bullet}\left(\oplus_{\alpha \in \Delta} \mathfrak{g}_{\alpha}\right)$ admits a canonical degree $(+1)$ derived Poisson algebra structure, where the multiplication is the wedge product, and the shifted $L_{\infty}$-brackets are given as follows:

(1) The unary bracket is the Chevalley-Eilenberg differential

$$
d_{\mathfrak{h}}: \Lambda^{\bullet} \mathfrak{h}^{\vee} \otimes \Lambda^{\bullet}\left(\oplus_{\alpha \in \Delta} \mathfrak{g}_{\alpha}\right) \rightarrow \Lambda^{\bullet+1} \mathfrak{h}^{\vee} \otimes \Lambda^{\bullet}\left(\oplus_{\alpha \in \Delta} \mathfrak{g}_{\alpha}\right) .
$$

Here the $\mathfrak{h}$-module structure on $\Lambda^{\bullet}\left(\oplus_{\alpha \in \Delta} \mathfrak{g}_{\alpha}\right)$ is the natural extension of the $\mathfrak{h}$-action on $\oplus_{\alpha \in \Delta} \mathfrak{g}_{\alpha}$.

${ }^{2}$ This means that the differential $d_{A}^{\nabla}$ is compatible with the Gerstenhaber bracket:

$$
d_{A}^{\nabla}\{X, Y\}=\left\{d_{A}^{\nabla} X, Y\right\}+(-1)^{|X|+1}\left\{X, d_{A}^{\nabla} Y\right\}, \quad \forall X, Y \in \Omega_{A}^{\bullet}\left(\Lambda^{\bullet} B\right) .
$$


(2) The binary bracket $\{-,-\}_{2}$ is generated by

$$
\left\{x_{\alpha}, x_{\beta}\right\}_{2}=c_{\alpha, \beta} x_{\alpha+\beta}, \quad \forall \alpha, \beta \in \Delta, \alpha+\beta \in \Delta .
$$

Here $c_{\alpha, \beta}$ is the standard structure constant (see [22, Chapter VII]).

(3) The ternary bracket $\{-,-,-\}_{3}$ is generated by

$$
\left\{x_{\alpha}, x_{-\alpha}, \xi\right\}_{3}=\left\langle h_{\alpha}, \xi\right\rangle, \quad \forall \xi \in \mathfrak{h}^{\vee},
$$

with all other situations being trivial.

(4) All higher brackets vanish.

Theorem 4.23. Let $\mathfrak{g}=\mathfrak{h} \oplus \bigoplus_{\alpha \in \Delta} \mathfrak{g}_{\alpha}$ be a complex semisimple Lie algebra, the Chevalley-Eilenberg hypercohomology $\mathbb{H}\left(\Lambda^{\bullet} \mathfrak{h} \vee \otimes \Lambda^{\bullet}\left(\oplus_{\alpha \in \Delta} \mathfrak{g}_{\alpha}\right), d_{\mathfrak{h}}\right)$ admits a canonical Gerstenhaber algebra structure.

4.4.3. Transitive Lie algebroids. Let $\left(L,[-,-]_{L}, \rho_{L}\right)$ be a transitive Lie algebroid over $M$, i.e. $\rho_{L}: L \rightarrow T_{M}$ is surjective. Let $\mathfrak{K}=\operatorname{ker} \rho_{L}$ be the adjoint bundle of $L$, which is a Lie algebra bundle over $M$. Then $(L, \mathfrak{K})$ is a Lie pair. The quotient $L / \mathfrak{K} \cong T_{M}$ has the trivial $\mathfrak{K}$-module structure.

Following Mackenzie [34], a connection is a bundle map $\gamma: T_{M} \rightarrow L$ such that $\rho_{L} \circ \gamma=\mathrm{id}_{T_{M}}$. A connection always exists. The curvature of $\gamma$ is the bundle map $R: \wedge^{2} T_{M} \rightarrow \mathfrak{K}$ :

$$
R(X, Y)=[\gamma(X), \gamma(Y)]_{L}-\gamma[X, Y], \quad \forall X, Y \in \mathscr{X}(M) .
$$

There also induces a $T_{M}$-connection on $\mathfrak{K}$ defined by

$$
\nabla_{X} u=[\gamma(X), u]_{L}, \quad \forall X \in \mathscr{X}(M), u \in \Gamma(\mathfrak{K}) .
$$

By choosing such a connection, $L$ can be identified with $\mathfrak{K} \oplus T_{M}$ as a vector bundle over $M$. The Lie algebroid structure on $L$ can be described as follows: the anchor is the projection to the second component, and the Lie bracket is

$$
[(u, X),(v, Y)]_{L}=\left(R(X, Y)+[u, v]_{\mathfrak{K}}+\nabla_{X} v-\nabla_{Y} u,[X, Y]\right), \quad \forall u, v \in \Gamma(\mathfrak{K}), X, Y \in \mathscr{X}(M) .
$$

Now for the Lie pair $(L, \mathfrak{K})$, by Proposition 4.3, Theorems 1.1 and 1.2, we have

Theorem 4.24. Let $L$ be a transitive Lie algebroid over $M$ with anchor $\rho_{L}$, and $\mathfrak{K}=\operatorname{ker} \rho_{L}$. Then, up to degree $(+1)$ derived Poisson algebra isomorphisms whose first Taylor coefficient is the identity map, $\Gamma\left(\Lambda^{\bullet} \mathfrak{K}^{\vee} \otimes \mathscr{X}_{\text {poly }}^{\bullet}(M)\right)$ admits a unique degree $(+1)$ derived Poisson algebra structure, where the multiplication is the wedge product, and shifted $L_{\infty}$ brackets are given as follows:

(1) The unary bracket

$$
d: \Gamma\left(\Lambda^{\bullet} \mathfrak{K}^{\vee} \otimes \mathscr{X}_{\text {poly }}^{\bullet}(M)\right) \rightarrow \Gamma\left(\Lambda^{\bullet+1} \mathfrak{K}^{\vee} \otimes \mathscr{X}_{\text {poly }}^{\bullet}(M)\right)
$$

is equal to $d_{\mathfrak{K}} \otimes 1$, where $d_{\mathfrak{K}}: \Gamma\left(\Lambda^{\bullet} \mathfrak{K}^{\vee}\right) \rightarrow \Gamma\left(\Lambda^{\bullet+1} \mathfrak{K}^{\vee}\right)$ is the Chevalley-Eilenberg differential of $\mathfrak{K}$.

(2) The binary bracket is generated by the following relations:

$$
\begin{aligned}
& \{X, Y\}_{2}=[X, Y], \\
& \{X, \omega\}_{2}=\nabla_{X} \omega, \\
& \{\omega, \eta\}_{2}=0,
\end{aligned}
$$

$\forall X, Y \in \mathscr{X}(M), \omega, \eta \in \Gamma\left(\mathfrak{K}^{\vee}\right)$.

(3) The ternary bracket is generated by the following relations:

$$
\{X, Y, \omega\}_{3}=\left\langle R^{\gamma}(X, Y), \omega\right\rangle, \quad \forall X, Y \in \mathscr{X}(M), \omega \in \Gamma\left(\mathfrak{K}^{\vee}\right),
$$

and otherwise vanishes.

(4) All the higher brackets vanish.

Theorem 4.25. Under the same hypothesis as in Theorem 4.24, the space $\mathbb{H}_{\mathrm{CE}}\left(\mathfrak{K}, \mathscr{X}_{\text {poly }}(M)\right)$ admits a canonical Gerstenhaber algebra structure. 
4.4.4. Foliations. Consider a regular foliation $F$ on a smooth manifold $M$. Then $\left(T_{M}, T_{F}\right)$ is a Lie pair, where $T_{F}$ is the integrable distribution corresponding to $F$. Let $N_{F}=T_{M} / T_{F}$ be the associated normal bundle. Applying Theorems 1.1 and 1.2, we have

Theorem 4.26. Let $F$ be a regular foliation on $M$. Then, up to degree $(+1)$ derived Poisson algebra isomorphisms whose first Taylor coefficient is the identity map, tot $\Gamma\left(\Lambda^{\bullet} T_{F}^{\vee} \otimes \Lambda^{\bullet} N_{F}\right)$ admits a canonical degree $(+1)$ derived Poisson algebra structure, where the multiplication is the wedge product, and the shifted $L_{\infty}$ brackets are given as follows:

(1) The unary bracket is the leafwise de Rham differential

$$
d_{d R}^{F}: \Gamma\left(\Lambda^{\bullet} T_{F}^{\vee} \otimes \Lambda^{\bullet} N_{F}\right) \rightarrow \Gamma\left(\Lambda^{\bullet+1} T_{F}^{\vee} \otimes \Lambda^{\bullet} N_{F}\right) .
$$

(2) Choose a complementary subbundle to $T_{F}$ in $T_{M}$ so that $T_{M} \cong T_{F} \oplus N_{F}$. The binary bracket

$$
\{-,-\}_{2}: \Gamma\left(\Lambda^{i} T_{F}^{\vee} \otimes \Lambda^{j} N_{F}\right) \times \Gamma\left(\Lambda^{p} T_{F}^{\vee} \otimes \Lambda^{l} N_{F}\right) \rightarrow \Gamma\left(\Lambda^{i+p} T_{F}^{\vee} \otimes \Lambda^{j+l-1} N_{F}\right)
$$

is generated by the following relations:

a) $\{u, v\}_{2}=\operatorname{pr}_{N_{F}}[u, v], \quad \forall u, v \in \Gamma\left(N_{F}\right)$;

b) $\{u, \omega\}_{2}=\operatorname{pr}_{T_{F}^{\vee}}\left(L_{u} \omega\right), \quad \forall u \in \Gamma\left(N_{F}\right), \omega \in \Gamma\left(T_{F}^{\vee}\right)$;

c) $\{u, f\}_{2}=u(f), \quad \forall u \in \Gamma\left(N_{F}\right), f \in C^{\infty}(M)$;

d) $\left\{\omega_{1}, \omega_{2}\right\}_{2}=0, \quad \forall \omega_{1}, \omega_{2} \in \Gamma\left(\Lambda^{\bullet} T_{F}^{\vee}\right)$.

(3) The ternary bracket

$\{-,-,-\}_{3}: \Gamma\left(\Lambda^{i} T_{F}^{\vee} \otimes \Lambda^{j} N_{F}\right) \times \Gamma\left(\Lambda^{p} T_{F}^{\vee} \otimes \Lambda^{l} N_{F}\right) \times \Gamma\left(\Lambda^{r} T_{F}^{\vee} \otimes \Lambda^{s} N_{F}\right) \rightarrow \Gamma\left(\Lambda^{i+p+r-1} T_{F}^{\vee} \otimes \Lambda^{j+l+s-2} N_{F}\right)$

is $C^{\infty}(M)$-linear in each entry and generated by the following relations:

a) $\{-,-,-\}_{3}$ vanishes when being restricted to $\Gamma\left(N_{F}\right) \times \Gamma\left(N_{F}\right) \times \Gamma\left(N_{F}\right), \Gamma\left(N_{F}\right) \times \Gamma\left(T_{F}^{\vee}\right) \times$ $\Gamma\left(T_{F}^{\vee}\right)$, and $\Gamma\left(T_{F}^{\vee}\right) \times \Gamma\left(T_{F}^{\vee}\right) \times \Gamma\left(T_{F}^{\vee}\right)$;

b) $\{u, v, \omega\}_{3}=\left\langle\operatorname{pr}_{T_{F}}[u, v], \omega\right\rangle$, for all $u, v \in \Gamma\left(N_{F}\right)$ and $\omega \in \Gamma\left(T_{F}^{\vee}\right)$.

(4) All the rest of higher brackets vanish.

Theorem 4.27. Let $F$ be a regular foliation on $M$. The leafwise de Rham hypercohomology $\mathbb{H}_{\mathrm{dR}}\left(F, \Lambda^{\bullet} N_{F}\right)$ admits a canonical Gerstenhaber algebra structure.

Remark 4.28. According to Proposition 4.1, a choice of a complementary subbundle to $T_{F}$ in $T_{M}$ induces an $L_{\infty}$ algebroid structure on $T_{F}[1] \oplus N_{F} \rightarrow T_{F}[1]$. In terms of purely algebraic term, this amounts to saying that the pair $\left(\Gamma\left(\Lambda^{\bullet} T_{F}^{\vee}\right), \Gamma\left(\Lambda^{\bullet} T_{F}^{\vee} \otimes N_{F}\right)\right)$ is an $L R_{\infty}$ algebra. This result is due to Vitagliano [52].

\section{Appendix A. $L_{\infty}$ ALgebroids AND DG MANIFOLDS}

We recall some standard notions and results which we used throughout the paper. We mainly follow the conventions of Bruce [8], Voronov [55], and Lada-Markl [28].

The following proposition reveals a close relation between $L_{\infty}$ algebroids and dg manifolds. See [8, 25].

Proposition A.1. Let $\mathcal{L} \rightarrow \mathcal{M}$ be a vector bundle of $\mathbb{Z}$-graded manifolds. Then $\mathcal{L}$ is an $L_{\infty}$ algebroid if and only if $\mathcal{L}[1]$ is a dg manifold whose homological vector field $Q$ is tangent to the zero section $\mathcal{M} \stackrel{0}{\hookrightarrow} \mathcal{L}[1]$.

The rest of the section is devoted to the proof of this proposition, where the set-up was used in the paper. We first start with the following

Lemma A.2. Let $\mathcal{L} \rightarrow \mathcal{M}$ be a vector bundle of $\mathbb{Z}$-graded manifolds, and let $k$ be a fixed integer. An $L_{\infty}$ algebroid structure on $\mathcal{L} \rightarrow \mathcal{M}$ is equivalent to an $L_{\infty}$ algebra structure on the $\mathbb{K}$-vector space $\Gamma(\mathcal{L}) \oplus$ $C^{\infty}(\mathcal{M})[k]$ with structure maps

$$
\bar{\lambda}_{l}: \Lambda^{l}\left(\Gamma(\mathcal{L}) \oplus C^{\infty}(\mathcal{M})[k]\right) \rightarrow\left(\Gamma(\mathcal{L}) \oplus C^{\infty}(\mathcal{M})[k]\right)[2-l]
$$


satisfying the Leibniz rule

$$
\begin{aligned}
\bar{\lambda}_{l}\left(w_{1}, w_{2}, \cdots, w_{l-1}, f w_{l}\right)= & \bar{\lambda}_{l}\left(w_{1}, w_{2}, \cdots, w_{l-1}, f\right) w_{l} \\
& +(-1)^{\left(l+\left|w_{1}\right|+\cdots+\left|w_{l-1}\right|\right)|f|} f \bar{\lambda}_{l}\left(w_{1}, w_{2}, \cdots, w_{l-1}, w_{l}\right),
\end{aligned}
$$

for all $w_{1}, \cdots, w_{l} \in \Gamma(\mathcal{L}) \oplus C^{\infty}(\mathcal{M})[k]$, and $f \in C^{\infty}(\mathcal{M})$, and the following conditions:

(1) $\Gamma(\mathcal{L})$ is an $L_{\infty}$ subalgebra of $\Gamma(\mathcal{L}) \oplus C^{\infty}(\mathcal{M})[k]$;

(2) $C^{\infty}(\mathcal{M})[k]$ is an $L_{\infty}$ ideal of $\Gamma(\mathcal{L}) \oplus C^{\infty}(\mathcal{M})[k]$, i.e. $\bar{\lambda}_{l}\left(w_{1}, w_{2}, \cdots, w_{l}\right) \in C^{\infty}(\mathcal{M})[k]$ if at least one of the arguments $w_{1}, w_{2}, \ldots, w_{l}$ is in $C^{\infty}(\mathcal{M})[k]$; and

(3) $C^{\infty}(\mathcal{M})[k]$ is abelian, i.e. $\bar{\lambda}_{l}\left(w_{1}, w_{2}, \cdots, w_{l}\right)=0$ if at least two of the arguments $w_{1}, w_{2}, \ldots, w_{l}$ are in $C^{\infty}(\mathcal{M})[k]$.

Proof. Assume that $\mathcal{L}$ is an $L_{\infty}$ algebroid with multi-brackets $\left(\lambda_{l}\right)_{l \geq 1}$ and multi-anchor maps $\left(\rho_{l}\right)_{l \geq 0}$ as in Definition 3.6. Define a sequence $\left(\bar{\lambda}_{l}\right)_{l \geq 1}$ of $\mathbb{K}$-multilinear maps

$$
\bar{\lambda}_{l}: \Lambda^{l}\left(\Gamma(\mathcal{L}) \oplus C^{\infty}(\mathcal{M})[k]\right) \rightarrow\left(\Gamma(\mathcal{L}) \oplus C^{\infty}(\mathcal{M})[k]\right)[2-l]
$$

by the following relations:

- $\bar{\lambda}_{l}\left(x_{1}, \cdots, x_{l}\right)=0$ if at least two of the arguments $x_{1}, \ldots, x_{l}$ are in $C^{\infty}(\mathcal{M})[k]$;

- $\bar{\lambda}_{l}\left(a_{1}, \cdots, a_{l}\right)=\lambda_{l}\left(a_{1}, \cdots, a_{l}\right)$, for all $a_{1}, \cdots, a_{l} \in \Gamma(\mathcal{L})$; and

- $\bar{\lambda}_{l+1}\left(a_{1}, \cdots, a_{l}, f\right)=\rho_{l}\left(a_{1}, \cdots, a_{l}\right) f$, for all $a_{1}, \cdots, a_{l} \in \Gamma(\mathcal{L})$ and $f \in C^{\infty}(\mathcal{M})[k]$.

It is straightforward to verify that $\left(\bar{\lambda}_{l}\right)_{l \geq 1}$ satisfy all the required properties.

The converse can be proved by going backwards.

Given a vector bundle $\mathcal{E} \stackrel{\pi}{\rightarrow} \mathcal{M}$ of $\mathbb{Z}$-graded manifolds, consider the graded Lie algebra $\mathscr{D} \leqslant 1(\mathcal{E})$ of first-order differential operators on $\mathcal{E}$. It can be identified to $\mathscr{X}(\mathcal{E}) \oplus C^{\infty}(\mathcal{E})$ in a canonical way. Since $C^{\infty}(\mathcal{E}) \cong$ $\Gamma\left(\hat{S}\left(\mathcal{E}^{\vee}\right)\right)$, the contraction operator $\iota_{s}$ by a section $s \in \Gamma(\mathcal{E})$ defines a derivation of $C^{\infty}(\mathcal{E})$, i.e. a vector field on $\mathcal{E}$. The inclusion $\Gamma(\mathcal{E}) \oplus C^{\infty}(\mathcal{M}) \hookrightarrow \mathscr{X}(\mathcal{E}) \oplus C^{\infty}(\mathcal{E})$ sending $s+f$ to $\iota_{s}+\pi^{*}(f)$ embeds $\Gamma(\mathcal{E}) \oplus C^{\infty}(\mathcal{M})$ into an abelian Lie subalgebra of $\mathscr{D} \leqslant 1(\mathcal{E})$. We proceed to define a projection $P: \mathscr{D} \leqslant 1(\mathcal{E}) \rightarrow \Gamma(\mathcal{E}) \oplus C^{\infty}(\mathcal{M})$. Given a vector field $X \in \mathscr{X}(\mathcal{E})$, consider the composition

$$
\Gamma\left(\mathcal{E}^{\vee}\right) \hookrightarrow \Gamma\left(\hat{S}\left(\mathcal{E}^{\vee}\right)\right) \cong C^{\infty}(\mathcal{E}) \stackrel{X}{\rightarrow} C^{\infty}(\mathcal{E}) \stackrel{0^{*}}{\rightarrow} C^{\infty}(\mathcal{M}),
$$

where $0^{*}$ denotes the pullback of functions through the zero section of the vector bundle $\mathcal{M} \stackrel{0}{\hookrightarrow} \mathcal{L}$. There exists a unique $X^{\uparrow} \in \Gamma(\mathcal{E})$ such that $\left\langle\xi, X^{\uparrow}\right\rangle=0^{*}(X(\xi))$, for all $\xi \in \Gamma\left(\mathcal{E}^{\vee}\right)$. Define $P: \mathscr{D}^{\leqslant 1}(\mathcal{E}) \rightarrow$ $\Gamma(\mathcal{E}) \oplus C^{\infty}(\mathcal{M})$ by $P(X+f)=X^{\uparrow}+0^{*}(f)$, for all $X \in \mathscr{X}(\mathcal{E})$ and $f \in C^{\infty}(\mathcal{E})$. Note that the projection operator $P$ satisfies

$$
P([x, y])=P([P(x), y]+[x, P(y)]), \quad \forall x, y \in \mathscr{D}^{\leqslant 1}(\mathcal{E}) .
$$

The following lemma is easily verified, and is left to the reader.

Lemma A.3. For any $Q \in \mathscr{X}(\mathcal{E})$, the following assertions are equivalent.

(1) The vector field $Q$ is tangent to the zero section of $\mathcal{E} \stackrel{\pi}{\rightarrow} \mathcal{M}$.

(2) There exists a unique vector field $\Xi$ on $\mathcal{M}$ such that the diagram

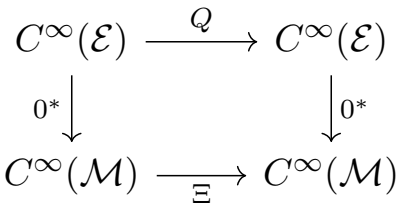

commutes. 
(3) The ideal $\operatorname{ker}\left(0^{*}\right)$ of $C^{\infty}(\mathcal{E})$ is $Q$-stable.

(4) $Q \in \operatorname{ker}(P)$

Proof of Proposition A.1. Consider the vector bundle $\mathcal{E} \stackrel{\pi}{\rightarrow} \mathcal{M}$ of $\mathbb{Z}$-graded manifolds, where $\mathcal{E}=\mathcal{L}[1]$. Assume that $Q$ is a homological vector field on $\mathcal{L}[1]$ tangent to the zero section of $\mathcal{L}[1] \stackrel{\pi}{\rightarrow} \mathcal{M}$. According to Lemma A.3, we have $Q \in \operatorname{ker}(P)$.

The graded Lie algebra $\mathfrak{A}=\mathscr{D}^{\leqslant 1}(\mathcal{L}[1])$, its abelian Lie subalgebra $\mathfrak{a}=\Gamma(\mathcal{L}[1]) \oplus C^{\infty}(\mathcal{M})$, the projection $P: \mathfrak{A} \rightarrow \mathfrak{a}$ of $\mathscr{D} \leqslant 1(\mathcal{L}[1])$ onto $\Gamma(\mathcal{L}[1]) \oplus C^{\infty}(\mathcal{M})$, and together with the vector field $Q \in \operatorname{ker}(P)$ constitute an $L_{\infty}[1]$ algebra Voronov data [55, Theorem 1 and Corollary 1]. The multibrackets $\left(\mu_{l}\right)_{l \geq 1}$ are given by a sequence of derived brackets:

$$
\mu_{l}\left(z_{1}, z_{2}, \cdots, z_{l}\right)=P\left(\left[\left[\left[\left[Q, z_{1}\right], z_{2}\right], \cdots\right], z_{l}\right]\right),
$$

for all $z_{1}, z_{2}, \ldots, z_{l} \in \Gamma(\mathcal{L}[1]) \oplus C^{\infty}(\mathcal{M})$.

Applying the décalage isomorphism, we obtain an $L_{\infty}$ algebra on $\Gamma(\mathcal{L}) \oplus C^{\infty}(\mathcal{M})[-1]$ with multibrackets $\left(\bar{\lambda}_{l}\right)_{l \geq 1}$. The multibrackets $\left(\mu_{l}\right)_{l \geq 1}$ and $\left(\bar{\lambda}_{l}\right)_{l \geq 1}$ are related as follows:

$$
\bar{\lambda}_{l}\left(w_{1}, w_{2}, \cdots, w_{l}\right)=(-1)^{\star} \mu_{l}\left(w_{1}, w_{2}, \cdots, w_{l}\right),
$$

where $\star=(l-1)\left|w_{1}\right|+(l-2)\left|w_{2}\right|+\cdots+\left|w_{l-1}\right|$ for all homogeneous $w_{1}, w_{2}, \ldots, w_{l} \in \Gamma(\mathcal{L}) \oplus C^{\infty}(\mathcal{M})[-1]$.

It is straightforward to verify that the $L_{\infty}$ algebra structure $\left(\bar{\lambda}_{l}\right)_{l \geq 1}$ on $\Gamma(\mathcal{L}) \oplus C^{\infty}(\mathcal{M})[-1]$ satisfies the four conditions listed in Lemma A.2. Therefore, $\mathcal{L} \rightarrow \mathcal{M}$ is an $L_{\infty}$ algebroid. Its multi-anchor maps $\rho_{l}: \Lambda^{l} \mathcal{L} \rightarrow$ $T_{\mathcal{M}}$ (with $l \geq 0$ ) and multi-brackets $\lambda_{l}: \Lambda^{l} \Gamma(\mathcal{L}) \rightarrow \Gamma(\mathcal{L})$ (with $l \geq 1$ ) are defined by the relations:

$$
\rho_{l}\left(a_{1}, a_{2}, \cdots, a_{l}\right) f=(-1)^{b} 0^{*}\left(\left[\left[\left[\left[Q, \iota_{a_{1}}\right], \iota_{a_{2}}\right], \cdots\right], \iota_{a_{l}}\right]\left(\pi^{*} f\right)\right),
$$

where $b=l\left|a_{1}\right|+(l-1)\left|a_{2}\right|+\cdots+\left|a_{l}\right|$ and

$$
\left\langle\lambda_{l}\left(a_{1}, a_{2}, \cdots, a_{l}\right), \xi\right\rangle=(-1)^{\sharp} 0^{*}\left(\left[\left[\left[\left[Q, \iota_{a_{1}}\right], \iota_{a_{2}}\right], \cdots\right], \iota_{a_{l}}\right](\xi)\right)
$$

where $\sharp=(l-1)\left|a_{1}\right|+(l-2)\left|a_{2}\right|+\cdots+\left|a_{l-1}\right|$ for all $\xi \in \Gamma\left(\mathcal{L}^{\vee}\right), a_{1}, a_{2}, \ldots, a_{l} \in \Gamma(\mathcal{L})$ and $f \in C^{\infty}(\mathcal{M})$.

Conversely, given an $L_{\infty}$ algebroid $\mathcal{L} \rightarrow \mathcal{M}$ with multi-anchors $\left(\rho_{l}\right)_{l \geq 0}$ and multibrackets $\left(\lambda_{l}\right)_{l \geq 1}$, one can recover the corresponding homological vector field $Q$ on $\mathcal{L}[1]$ satisfying the desired properties.

The algebra $C^{\infty}(\mathcal{L}[1])$ admits the direct product decomposition

$$
C^{\infty}(\mathcal{L}[1])=\prod_{k=0}^{\infty} \Gamma\left(S^{k}\left(\mathcal{L}^{\vee}[-1]\right)\right)
$$

We will refer to $\Gamma\left(S^{k}\left(\mathcal{L}^{\vee}[-1]\right)\right)$ as the weight $k$ component of $C^{\infty}(\mathcal{L}[1])$. Note that $\pi^{*} C^{\infty}(\mathcal{M})$ (the component of weight 0$)$ and $\Gamma\left(\mathcal{L}^{\vee}[-1]\right)$ (the component of weight 1$)$ generate the associative algebra $C^{\infty}(\mathcal{L}[1])$. A vector field $Q$ on $\mathcal{L}[1]$ is necessarily of the form $Q=\sum_{l=-1}^{\infty} D_{l}$, where $D_{l}$ is a derivation on $C^{\infty}(\mathcal{L}[1])$ of weight $l$ :

$$
D_{l}: \Gamma\left(\hat{S}^{\bullet}\left(\mathcal{L}^{\vee}[-1]\right)\right) \rightarrow \Gamma\left(\hat{S}^{\bullet+l}\left(\mathcal{L}^{\vee}[-1]\right)\right) .
$$

Since $Q$ is tangent to $\mathcal{M}$, i.e. we want $Q \in \operatorname{ker}(P)$, its weight $(-1)$ component $D_{-1}$ must vanish. Choose a local coordinate chart $\left(x^{j}\right)_{j \in J}$ on $\mathcal{M}$; a local frame $\left(s_{k}\right)_{k \in K}$ for $\mathcal{L}[1]$; and the dual local frame $\left(\xi^{k}\right)_{k \in K}$ for $\mathcal{L}^{\vee}[-1]$, the derivation $D_{l}$ can be written as

$$
D_{l}=\frac{1}{l !} \sum_{j \in J} \xi^{i_{l}} \cdots \xi^{i_{2}} \xi^{i_{1}} \pi^{*}\left(\tilde{Q}_{i_{1}, i_{2}, \cdots, i_{l}}^{j}\right) \frac{\partial}{\partial x^{j}}+\frac{1}{(l+1) !} \sum_{k \in K} \xi^{i_{l}} \cdots \xi^{i_{1}} \xi^{i_{0}} \pi^{*}\left(Q_{i_{0}, i_{1}, \cdots, i_{l}}^{k}\right) \frac{\partial}{\partial \xi^{k}},
$$

where

$$
\tilde{Q}_{i_{1}, i_{2}, \cdots, i_{l}}^{j}=(-1)^{\diamond} \rho_{l}\left(s_{i_{1}}, s_{i_{2}}, \cdots, s_{i_{l}}\right) x_{j}
$$


with $\diamond=(l-1)\left|s_{i_{1}}\right|+(l-2)\left|s_{i_{2}}\right|+\cdots+\left|s_{i_{l-1}}\right|$, and

$$
Q_{i_{0}, i_{1}, \cdots, i_{l}}^{k}=(-1)^{\dagger}\left\langle\lambda_{l+1}\left(s_{i_{0}}, s_{i_{1}}, \cdots, s_{i_{l}}\right), \xi^{k}\right\rangle
$$

with $\dagger=(l+1)\left|s_{i_{0}}\right|+l\left|s_{i_{1}}\right|+\cdots+\left|s_{i_{l}}\right|$. One checks that $D_{l}$, and therefore $Q$, is well defined. In summary, the multi-anchors and multi-brackets of the $L_{\infty}$ algebroid $\mathcal{L} \rightarrow \mathcal{M}$ determine through Equations (37) and (38) a vector field $Q$ of degree +1 on $\mathcal{L}[1]$, which is tangent to the zero section $\mathcal{M}$.

The $L_{\infty}[1]$ algebra structure $\left(\mu_{l}\right)_{l \geq 1}$ on $\Gamma(\mathcal{L}[1]) \oplus C^{\infty}(\mathcal{M})$ determined by the $L_{\infty}$ algebroid $\mathcal{L} \rightarrow \mathcal{M}$ as per Lemma A.2 is related to the vector field $Q$ through Equation (36). It follows from the generalized Jacobi identity, Equation (36), and repetitive use of Equation (35) that

$$
0=\sum_{p+r=l+1}\left(\mu_{p} \circ \mu_{r}\right)\left(z_{1}, \cdots, z_{l}\right)=P\left(\left[\left[\left[\left[[Q, Q], z_{1}\right], z_{2}\right], \cdots\right], z_{l}\right]\right),
$$

for all $z_{1}, z_{2}, \ldots, z_{l} \in \Gamma(\mathcal{L}[1]) \oplus C^{\infty}(\mathcal{M})$. Therefore $[Q, Q]=0$, i.e. $Q$ is homological.

\section{Appendix B. Shifted Poisson algebras}

Definition B.1. A degree $k$ Poisson algebra is a $\mathbb{Z}$-graded commutative and associative algebra $\mathfrak{R}$ with a degree $k$ Poisson bracket, denoted by $[-,-]\left(\right.$ i.e. $\left.\left[\mathfrak{R}^{i}, \mathfrak{R}^{j}\right] \subset \mathfrak{R}^{i+j+k}\right)$, satisfying

(1) $[a, b]=-(-1)^{|a|^{[k]}|b|^{[k]}}[b, a]$,

(2) $[a,[b, c]]=[[a, b], c]+(-1)^{|a|^{[k]}|b|^{[k]}}[b,[a, c]]$,

(3) $[a, b c]=[a, b] c+(-1)^{|a|^{[k]}|b|} b[a, c]$,

for all homogeneous elements $a, b, c \in \mathfrak{R}$.

Note that Conditions (1) and (2) are equivalent to that $\mathfrak{R}[-k]$ is a $\mathbb{Z}$-graded Lie algebra, while (3) means that the Lie bracket is a biderivation.

Also note that degree 0 Poisson algebras are usual Poisson algebras, and degree $(+1)$ Poisson algebras are Gerstenhaber algebras. In the meantime, one can obtain a Poisson algebra of degree $k$ out of a graded Lie algebra as indicated in the following

Proposition B.2. Let $\mathfrak{g}$ be a graded Lie algebra. Then the symmetric product $S(\mathfrak{g}[k])$ (similarly $\hat{S}(\mathfrak{g}[k])$ ) admits a unique degree $k$ Poisson algebra structure which extends the original Lie bracket on $\mathfrak{g}$.

\section{ApPENdix C. Shifted POLYVeCtor FIELDS}

Let $\mathcal{M}$ be a $\mathbb{Z}$-graded manifold. A degree $l$ vector field $X \in \mathscr{X}(\mathcal{M})$ is a derivation $C^{\infty}(\mathcal{M}) \stackrel{X}{\rightarrow} C^{\infty}(\mathcal{M})$ of degree $l$. The degree of $X$ is denoted by $|X|=l$.

The commutator in $\mathscr{X}(\mathcal{M})$ is standard:

$$
[X, Y]=X \circ Y-(-1)^{|X||Y|} Y \circ X,
$$

for all homogeneous $X, Y \in \mathscr{X}(\mathcal{M})$. It is obvious that $\mathscr{X}(\mathcal{M})$ is a left $C^{\infty}(\mathcal{M})$-module, and the pair $\left(\mathscr{X}(\mathcal{M}), C^{\infty}(\mathcal{M})\right)$ forms a $\mathbb{Z}$-graded Lie-Rinehart algebra.

Let $n \in \mathbb{Z}$ be a fixed integer. Following Pridham [39,40], let $\mathscr{X}_{\text {poly }}^{0}(\mathcal{M}, n)=C^{\infty}(\mathcal{M})$, and for each $m \geq 1$,

$$
\mathscr{X}_{\text {poly }}^{m}(\mathcal{M}, n)=S_{C^{\infty}(\mathcal{M})}^{m}\left(\Gamma\left(\mathcal{M} ; T_{\mathcal{M}}[n+1]\right) .\right.
$$

Elements in $\mathscr{X}_{\text {poly }}^{m}(\mathcal{M}, n)$ are called $n$-shifted $m$-polyvector fields on $\mathcal{M}$. Then the space

$$
\mathscr{X}_{\text {poly }}^{\bullet}(\mathcal{M}, n)=\oplus_{m \geq 0} \mathscr{X}_{\text {poly }}^{m}(\mathcal{M}, n)
$$


is called the $n$-shifted Schouten-Nijenhuis algebra of $\mathcal{M}$. Its completion is denoted by $\hat{\mathscr{X}}_{\text {poly }}^{\bullet}(\mathcal{M}, n)$. It is simple to see that

$$
\hat{\mathscr{X}}_{\text {poly }}^{\bullet}(\mathcal{M}, n) \cong C^{\infty}\left(T_{\mathcal{M}}^{\vee}[-n-1]\right),
$$

the space of functions on the $(-n-1)$-shifted cotangent bundle $T_{\mathcal{M}}^{\vee}$.

Since $T_{\mathcal{M}}$ is a Lie algebroid, $T_{\mathcal{M}}^{\vee}$ is a canonical Poisson manifold, which is in fact symplectic. According to Proposition 3.8, we have the following

Lemma C.1. The space $\mathscr{X}_{\text {poly }}^{\bullet}(\mathcal{M}, n)$ admits a degree $(n+1)$ Poisson algebra structure, similarly $\hat{\mathscr{X}}_{\text {poly }}^{\bullet}(\mathcal{M}, n)$.

This degree $(n+1)$ Poisson bracket is also known as the $n$-shifted Schouten-Nijenhuis bracket. When $n=0$, the space of 0 -shifted polyvector fields $\mathscr{X}_{\text {poly }}^{\bullet}(\mathcal{M}, 0)$, coincides with the usual Schouten-Nijenhuis algebra on $\mathcal{M}$, which is simply denoted by $\mathscr{X}_{\text {poly }}^{\bullet}(\mathcal{M})$. When $n=-1$, the space of $(-1)$-shifted polyvector fields $\mathscr{X}_{\text {poly }}^{\bullet}(\mathcal{M},-1)$ is the Poisson algebra $\operatorname{Pol}\left(T_{\mathcal{M}}^{\vee}\right)$. Its completion $\hat{\mathscr{X}}_{\text {poly }}^{\bullet}(\mathcal{M},-1) \cong C^{\infty}\left(T_{\mathcal{M}}^{\vee}\right)$.

Any element in $\mathscr{X}_{\text {poly }}^{m}(\mathcal{M}, n)$ is a finite sum of homogeneous elements of the form:

$$
\Pi=\bar{X}_{1} \odot \bar{X}_{2} \odot \cdots \odot \bar{X}_{m},
$$

where $X_{i} \in \mathscr{X}(\mathcal{M})$, and $\bar{X}_{i} \in \mathscr{X}(\mathcal{M})[n+1]$ denotes the corresponding element with shifted degree. The number $|\Pi|=\left|X_{1}\right|+\cdots+\left|X_{m}\right|$ is called the pure degree of $X$, whereas $m$ is called the weight. By

$$
\|\Pi\|_{n}=|\Pi|-m(n+1),
$$

we denote the total degree of $\Pi$. The following lemma provides an alternative description of shifted polyvector fields.

Lemma C.2. A homogeneous $n$-shifted m-polyvector field $\Pi$ on $\mathcal{M}$ is equivalently to a $m$-ary operation of degree $|\Pi|$ (the pure degree of $\Pi$ ):

$$
\Pi:\left(C^{\infty}(\mathcal{M})\right)^{\otimes m} \rightarrow C^{\infty}(\mathcal{M})
$$

satisfying the following properties:

1) $\Pi$ is symmetric multilinear on $C^{\infty}(\mathcal{M})[-n-1]$ :

$$
\Pi\left(f_{1}, \cdots, f_{i-1}, f_{i}, f_{i+1}, f_{i+2}, \cdots, f_{m}\right)=(-1)^{\left|f_{i}\right|^{[n+1]}\left|f_{i+1}\right|^{[n+1]}} \Pi\left(f_{1}, \cdots, f_{i-1}, f_{i+1}, f_{i}, f_{i+1}, \cdots, f_{m}\right) \text {; }
$$

2) $\Pi$ is a derivation of degree $|\Pi|$ :

$$
\begin{aligned}
& \Pi\left(f_{1}, \cdots, f_{m-1}, f_{m} f_{m}^{\prime}\right) \\
= & \Pi\left(f_{1}, \cdots, f_{m-1}, f_{m}\right) f_{m}^{\prime}+(-1)^{\left(|\Pi|+\left|f_{1}\right|+\cdots+\left|f_{m-1}\right|\right)\left|f_{m}\right|} f_{m} \Pi\left(f_{1}, \cdots, f_{m-1}, f_{m}^{\prime}\right) .
\end{aligned}
$$

The proof is omitted as it is completely analogous to the usual unshifted polyvector fields on ordinary smooth manifolds.

Finally, we need a technical lemma for an explicit formula describing the $(n+1)$-shifted Poisson bracket in $\mathscr{X}_{\text {poly }}^{\bullet}(\mathcal{M}, n)$. For any $\Pi \in \mathscr{X}_{\text {poly }}^{p}(\mathcal{M}, n)$ and $\Lambda \in \mathscr{X}_{\text {poly }}^{q}(\mathcal{M}, n)$, let $\Pi \circ \Lambda$ be the $(p+q-1)$-ary operation $\left(C^{\infty}(\mathcal{M})\right)^{\otimes p+q-1} \rightarrow C^{\infty}(\mathcal{M})$ given by

$$
=\sum_{\sigma \in \operatorname{Sh}(p, q-1)} \epsilon^{[n+1]}(\sigma) \Pi\left(\Lambda\left(f_{\sigma(1)}, \cdots, f_{\sigma(q)}\right), f_{\sigma(q+1)}, \cdots, f_{\sigma(p+q-1)}\right) .
$$

Here $\epsilon^{[n+1]}(\sigma)$ denotes the Koszul sign with respect to the shifted degrees $\left|f_{1}\right|^{[n+1]}, \cdots,\left|f_{p+q}\right|^{[n+1]}$.

Lemma C.3. For any $\Pi \in \mathscr{X}_{\text {poly }}^{p}(\mathcal{M}, n)$ and $\Lambda \in \mathscr{X}_{\text {poly }}^{q}(\mathcal{M}, n)$, the degree $(n+1)$ Poisson bracket $[\Pi, \Lambda]$ in $\mathscr{X}_{\text {poly }}^{\bullet}(\mathcal{M}, n)$ as in Lemma $C .1$ coincides with the graded commutator:

$$
[\Pi, \Lambda]=\Pi \circ \Lambda-(-1)^{\left(\|\Pi\|_{n}+n+1\right)\left(\|\Lambda\|_{n}+n+1\right)} \Lambda \circ \Pi .
$$




\section{AcKNowledgments}

We would like to thank Martin Bordemann, Oliver Elchinger, Camille Laurent-Gengoux, Pavol Ševera, Jim Stasheff, and Luca Vitagliano for fruitful discussions and useful comments.

\section{REFERENCES}

1. Ruggero Bandiera, Descent of Deligne-Getzler $\infty$-groupoids, ArXiv e-prints (2017).

2. Ruggero Bandiera, Mathieu Stiénon, and Ping Xu, Polyvector fields and polydifferential operators associated with Lie pairs, work in progress.

3. Denis Bashkirov and Alexander A. Voronov, The BV formalism for $L_{\infty}$-algebras, J. Homotopy Relat. Struct. 12 (2017), no. 2 , 305-327. MR 3654355

4. G. Bonavolontà and N. Poncin, On the category of Lie n-algebroids, J. Geom. Phys. 73 (2013), 70-90. MR 3090103

5. M. Bordemann, O. Elchinger, S. Gutt, and A. Makhlouf, L-infinity Formality check for the Hochschild Complex of Certain Universal Enveloping Algebras, ArXiv e-prints (2018).

6. Raoul Bott, Lectures on characteristic classes and foliations, Lectures on algebraic and differential topology (Second Latin American School in Math., Mexico City, 1971), Springer, Berlin, 1972, Notes by Lawrence Conlon, with two appendices by J. Stasheff, pp. 1-94. Lecture Notes in Math., Vol. 279. MR 0362335

7. Ronald Brown, The twisted Eilenberg-Zilber theorem, Simposio di Topologia (Messina, 1964), Edizioni Oderisi, Gubbio, 1965, pp. 33-37. MR 0220273 (36\#3339)

8. Andrew James Bruce, From $L_{\infty}$-algebroids to higher Schouten/Poisson structures, Rep. Math. Phys. 67 (2011), no. 2, $157-177$. MR 2840338

9. Alberto S. Cattaneo and Giovanni Felder, Relative formality theorem and quantisation of coisotropic submanifolds, Adv. Math. 208 (2007), no. 2, 521-548. MR 2304327

10. Zhuo Chen, Mathieu Stiénon, and Ping Xu, From Atiyah classes to homotopy Leibniz algebras, Comm. Math. Phys. 341 (2016), no. 1, 309-349. MR 3439229

11. V. A. Dolgushev, A. E. Hoffnung, and C. L. Rogers, What do homotopy algebras form?, ArXiv e-prints (2014).

12. V. A. Dolgushev and C. L. Rogers, A Version of the Goldman-Millson Theorem for Filtered L-infinity Algebras, ArXiv e-prints (2014).

13. Samuel Eilenberg and Saunders Mac Lane, On the groups of $H(\Pi, n)$. I, Ann. of Math. (2) 58 (1953), 55-106. MR 0056295 $(15,54 b)$

14. Domenico Fiorenza and Marco Manetti, $L_{\infty}$ structures on mapping cones, Algebra Number Theory 1 (2007), no. 3, 301-330. MR 2361936

15. M. Gualtieri, M. Matviichuk, and G. Scott, Deformation of Dirac structures via L_ $\infty$ algebras, ArXiv e-prints (2017).

16. V. K. A. M. Gugenheim, On the chain-complex of a fibration, Illinois J. Math. 16 (1972), 398-414. MR 0301736

17. V. K. A. M. Gugenheim, L. A. Lambe, and J. D. Stasheff, Perturbation theory in differential homological algebra. II, Illinois J. Math. 35 (1991), no. 3, 357-373. MR 1103672

18. Johannes Huebschmann, Higher homotopies and Maurer-Cartan algebras: quasi-Lie-Rinehart, Gerstenhaber, and BatalinVilkovisky algebras, The breadth of symplectic and Poisson geometry, Progr. Math., vol. 232, Birkhäuser Boston, Boston, MA, 2005, pp. 237-302. MR 2103009

19. Multi derivation Maurer-Cartan algebras and sh Lie-Rinehart algebras, J. Algebra 472 (2017), 437-479. MR 3584886

20. Johannes Huebschmann and Tornike Kadeishvili, Small models for chain algebras, Math. Z. 207 (1991), no. 2, $245-280$. MR 1109665

21. Johannes Huebschmann and James D. Stasheff, Formal solution of the master equation via HPT and deformation theory, Forum Math. 14 (2002), no. 6, 847-868. MR 1932522

22. J.E. Humphreys, Introduction to Lie algebras and representation theory, Graduate Texts in Mathematics, vol. 9, Springer-Verlag, New York-Berlin, 1978, Second printing, revised. MR 499562

23. Frank Keller and Stefan Waldmann, Formal deformations of Dirac structures, J. Geom. Phys. 57 (2007), no. 3, $1015-1036$. MR 2275207

24. H. Khudaverdian and Theodore T. Voronov, Higher Poisson brackets and differential forms, Geometric methods in physics, AIP Conf. Proc., vol. 1079, Amer. Inst. Phys., Melville, NY, 2008, pp. 203-215. MR 2757715

25. _ Thick morphisms, higher koszul brackets, and $L_{\infty}$-algebroids, ArXiv Mathematics e-print 1808.10049 (2014).

26. L.J. Kjeseth, BRST cohomology and homotopy Lie-Rinehart pairs, ProQuest LLC, Ann Arbor, MI, 1996, Thesis (Ph.D.)-The University of North Carolina at Chapel Hill. MR 2695305

27. __ Homotopy Rinehart cohomology of homotopy Lie-Rinehart pairs, Homology Homotopy Appl. 3 (2001), no. 1, $139-163$. MR 1854642

28. Tom Lada and Martin Markl, Strongly homotopy Lie algebras, Comm. Algebra 23 (1995), no. 6, 2147-2161. MR 1327129

29. H.L. Lang, Y.H. Sheng, and X.M. Xu, Strong homotopy Lie algebras, homotopy Poisson manifolds and Courant algebroids, Lett. Math. Phys. 107 (2017), no. 5, 861-885. MR 3633027 
30. Camille Laurent-Gengoux, Sylvain Lavau, and Thomas Strobl, The universal Lie $\infty$-algebroid over a singular foliation, 2017, work in progress.

31. Hsuan-Yi Liao, Mathieu Stiénon, and Ping Xu, Formality theorem for g-manifolds, C. R. Math. Acad. Sci. Paris 355 (2017), no. 5, 582-589. MR 3650387

32. Zhang-Ju Liu, Alan Weinstein, and Ping Xu, Manin triples for Lie bialgebroids, J. Differential Geom. 45 (1997), no. 3, $547-574$. MR 1472888

33. Jiang-Hua Lu, Poisson homogeneous spaces and Lie algebroids associated to Poisson actions, Duke Math. J. 86 (1997), no. 2, 261-304. MR 1430434

34. Kirill C. H. Mackenzie, General theory of Lie groupoids and Lie algebroids, London Mathematical Society Lecture Note Series, vol. 213, Cambridge University Press, Cambridge, 2005. MR 2157566

35. Kirill C. H. Mackenzie and Tahar Mokri, Locally vacant double Lie groupoids and the integration of matched pairs of Lie algebroids, Geom. Dedicata 77 (1999), no. 3, 317-330. MR 1716681

36. Marco Manetti, Lectures on deformations of complex manifolds (deformations from differential graded viewpoint), Rend. Mat. Appl. (7) 24 (2004), no. 1, 1-183. MR 2130146

37. Tahar Mokri, Matched pairs of Lie algebroids, Glasgow Math. J. 39 (1997), no. 2, 167-181. MR 1460632

38. Yong-Geun Oh and Jae-Suk Park, Deformations of coisotropic submanifolds and strong homotopy Lie algebroids, Invent. Math. 161 (2005), no. 2, 287-360. MR 2180451

39. J. P. Pridham, Shifted Poisson and symplectic structures on derived N-stacks, J. Topol. 10 (2017), no. 1, 178-210. MR 3653066

40. __ An outline of shifted Poisson structures and deformation quantisation in derived differential geometry, ArXiv e-prints (2018).

41. Pedro Real, Homological perturbation theory and associativity, Homology Homotopy Appl. 2 (2000), 51-88. MR 1782593

42. George S. Rinehart, Differential forms on general commutative algebras, Trans. Amer. Math. Soc. 108 (1963), $195-222$. MR 0154906 (27 \#4850)

43. Dmitry Roytenberg, Courant algebroids, derived brackets and even symplectic supermanifolds, ProQuest LLC, Ann Arbor, MI, 1999, Thesis (Ph.D.)-University of California, Berkeley. MR 2699145

44. __ Quasi-Lie bialgebroids and twisted Poisson manifolds, Lett. Math. Phys. 61 (2002), no. 2, 123-137. MR 1936572

45. H. Sati, U. Schreiber, and J.D. Stasheff, Twisted differential string and fivebrane structures, Comm. Math. Phys. 315 (2012), no. 1, 169-213. MR 2966944

46. Y.H. Sheng and C.C. Zhu, Higher extensions of Lie algebroids, Commun. Contemp. Math. 19 (2017), no. 3, $1650034,41$. MR 3631929

47. Weishu Shih, Homologie des espaces fibrés, Inst. Hautes Études Sci. Publ. Math. (1962), no. 13, 88. MR 0144348

48. Mathieu Stiénon and Ping Xu, Fedosov dg manifolds associated with Lie pairs, ArXiv e-prints (2016).

49. Pavol Ševera, private communication.

50. __ Letters to Alan Weinstein about Courant algebroids, ArXiv e-prints (2017).

51. Arkady Yu. Văntrob, Lie algebroids and homological vector fields, Uspekhi Mat. Nauk 52 (1997), no. 2(314), 161-162. MR 1480150

52. Luca Vitagliano, On the strong homotopy Lie-Rinehart algebra of a foliation, Commun. Contemp. Math. 16 (2014), no. 6, 1450007, 49. MR 3277952

53. On the strong homotopy associative algebra of a foliation, Commun. Contemp. Math. 17 (2015), no. 2, $1450026,34$. MR 3313214

54. __ Representations of homotopy Lie-Rinehart algebras, Math. Proc. Cambridge Philos. Soc. 158 (2015), no. 1, $155-191$. MR 3300319

55. Theodore T. Voronov, Higher derived brackets and homotopy algebras, J. Pure Appl. Algebra 202 (2005), no. 1-3, $133-153$. MR 2163405

56. __ Higher derived brackets for arbitrary derivations, Travaux mathématiques. Fasc. XVI, Trav. Math., vol. 16, Univ. Luxemb., Luxembourg, 2005, pp. 163-186. MR 2223157

57. __ Microformal geometry and homotopy algebras, ArXiv Mathematics e-print 1411.6720 (2014).

58. __ "Nonlinear pullbacks" of functions and $L_{\infty}$-morphisms for homotopy Poisson structures, J. Geom. Phys. 111 (2017), 94-110. MR 3575558 
Dipartimento di Matematica, Sapienza Università di Roma

E-mail address: bandiera@mat.uniroma1.it

Department of Mathematics, Tsinghua University

E-mail address: chenzhuo@tsinghua.edu.cn

Department of Mathematics, Pennsylvania State University

E-mail address: stienon@psu.edu

Department of Mathematics, Pennsylvania State University

E-mail address: ping@math.psu.edu 